Index of

Generic Names

of Fossil Plants,

$1966-1973$

GEOLOGICAL SURVEY BULLETIN 1396 



\section{Index of}

Generic Names

\section{of Fossil Plants,}

\section{6-1973}

By ANNA M. BLAZER

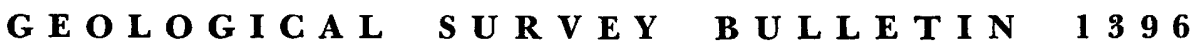

Based on the Compendium

Index of Paleobotany of

the U.S. Geological Survey

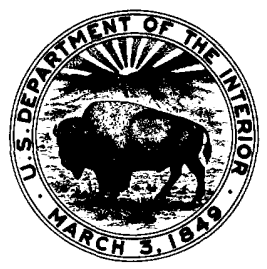




\section{UNITED STATES DEPARTMENT OF THE INTERIOR}

ROGERS C. B. MORTON, Secretary

GEOLOGICAL SURVEY

V. E. McKelvey, Director

Library of Congress Cataloging in Publication Data

Blazer, Anna M.

Index of generic names of fossil plants, 1966-1973.

(Geological Survey bulletin 1396)

"Based on the Compendium index of paleobotany of the U. S. Geological Survey."

Supplement to Index of generic names of fossil plants, 1820-1965, by H. N. Andrews.

Supt. of Docs. no.: I 19.3:1396

1. Paleobotany-Nomenclators. 2. Paleobotany-Bibliography I. Andrews, Henry Nathaniel, 1910-Index of generic names of fossil plants, 1820-1965. II. Title. III. Series: United States. Geological Survey. Bulletin, 1396. QE75.B9 no. 1396 [QE903] 557.3'08s [561'.1] 74-14995

For sale by the Superintendent of Documents, U.S. Government Printing Office Washington, D.C. 20402

Stock Number 024-001-02646-5 


\section{CONTENTS}

$\longrightarrow$

Page

Introduction -

Generic index of fossil plants 2

Bibliography -0.- 35 



\title{
INDEX OF GENERIC NAMES \\ OF FOSSIL PLANTS, 1966-1973
}

\author{
By Anna M. Blazer
}

\section{INTRODUCTION}

This bulletin is a supplement to the "Index of Generic Names of Fossil Plants, 1820-1965," by Henry N. Andrews, Jr. (U. S. Geol. Survey Bull. 1300, 1970). It includes the names of new fossil plant genera found in the literature during the 8-year period 1966-73, and now included in the U.S. Geological Survey's Compendium Index of Paleobotany. Also included are a few names published in earlier literature but not contained in Andrews' work. (For a complete history and background of the Compendium Index, see Andrews, 1970.)

This supplement follows the same general format used in the 1970 Index, except that the titles of Russian articles are both transliterated and translated. This makes for longer individual citations, but it also expedites access to the Russian literature.

Where more than one author has chosen the same name to describe a fossil plant, the publication bearing the earliest date is the one that has been selected for inclusion in this supplement.

The method followed in obtaining necessary data has been to visit regularly the local libraries to select paleobotanical material for scanning and inclusion of pertinent material in the Compendium Index. This involves searching through approximately 18,000 pieces of geologic literature yearly in the U.S. Geological Survey Library alone; even so, a few names are missed because of the growing mass of literature.

In the interest of maintaining a complete record of paleobotanical research in the Compendium Index, it is urgent that all paleobotanists send reprints or a notation of their publications to: 


\section{The Paleobotanical Library}

Paleontology and Stratigraphy Branch

U.S. Geological Survey

Room W-300, U.S. National Museum

Washington, D. C. 20244, U. S. A.

I have received help of many kinds during the preparation of the supplement, all of which is sincerely appreciated. Sergius $\mathrm{H}$. Mamay generally supervised this work; he and Francis M. Hueber of the Smithsonian Institution assisted me with problems of fossil identification and other technicalities. For assistance in library research I thank Marjorie Arnold and Arthur D. Watt. Birute Saldukas gave valuable help in linguistic problems. The librarians of the U.S. Geological Survey and the Smithsonian Institution gave generously of their time in locating publications. I owe special thanks to Marcia Lakeman of the Geological Society of America for her advice and help in cataloguing.

\section{GENERIC INDEX OF FOSSIL PLANTS}

A

AACHENIA Knobloch, 1972.

Aachenia debeyi Knobloch, 1972, p. 401-405, figs. 1-10; cone, Coniferales; Upper Cretaceous; Aachen, West Germany.

ABRUPTOPHYCUS Vologdin, 1962. Abruptophycus compositus Vologdin, 1962 , pt. 1, p. 232-235, figs. 41-43; pl. 41 , figs. $1-3$; pl. 42 , figs. 1,2 ; stromatolite, Vesiculariaceae; Sinian; right bank of the lower Nizhney Tunguska, about $8.5 \mathrm{~km}$ from the mouth, U.S.S.R.

ACACIELLA Walter, 1972.

Acaciella austrica (Howchin) Walter, 1972, p. 113-123, figs. 6, 21$22,29-32$; pl. 2 , figs. 3,4 ; pls. 13 14; pl. 15, figs. 1, 2; stromatolite; upper Riphean (Adelaidean); Northern Territory, Australia.

ACANTHINA Korde, 1973.

Acanthina multiformis Korde. 1973, p. 117 , pl. 4, fig. 2 ; pl. 5, figs. 1, 2 ; pl. 7, fig. 6 ; algea, Acanthinaseae; Lower Cambrian: Bazaikha river, eastern Sayan, U.S.S.S.R.

ACHLAMYDOCARPON'SchumadkerLambry, 1966.

Achlamydocarpon belgicum Schumacker-Lambry, 1966, p. 21-22, pls. 1-5, figs. 1-30: cone, Lepidocarpaceae; Upper Carboniferous; eastern Belgium.

ACTINIDIOXYLON Müller-Stoll and Mädel-Angeliewa, 1969.
Actinidioxylon princeps (Ludwig, 1860) Müller-Stoll and MädelAngeliewa, 1869. p. 103-108, figs. 1-3; pl. 1, figs. 1-4; pl. 2, figs. 69 ; fragments of woody twigs or branches; Pliocene; Dernbach in Westerwald, western Germany.

ACTINOXYLON Matten, 1968.

Actinoxylon banksii Matten, 1968, p. 776-779, figs. 1-18; progymnosperm; Middle Devonian; near Cairo, New York, U.S.A.

ADENANTHEROXYLON Prakash and Tripathi, 1968.

Adenantheroxylon pavoninium Prakash and Tripathi, 1968, p. 115 , figs. 1,2 ; wood, closely resembles the modern species Adenanthera pavonina, Leguminosae; Tertiary; Hailakandi, Cachar district, Assam, India.

AESCHYNOMENOXYLON MüllerStoll and Mädel, 1967.

Aeschynomenoxylon tertianum (Prakash) Müller-Stoll and Mädel, 1967, p. 149-150; wood, Leguminosae; lower Tertiary; India.

AFGHANOPOLIA Kaever, 1969.

Afghanopolia fragilis Kaever, 1969 , p. 26-30, figs. 6: 4-5. 7: 1-6; pl. 10, figs. 1-9; algae, Cymopolieae; middle Eocene; east Afghanistan.

AGAMUS Vologdin, 1970.

Agamus shungiticus Vologdin, 1970, p. 1165-1166, fig. 1v; unicellular 
miscroscopic algae, Vesiculariaceae; Precambrian; Shun'ga and Zaonezh'e villages, Karelo-Finnish SSR.

AGARDHIELLOPSIS Lemoine, 1966. Agardheillopsis cretacea Lemoine, 1966 , p. 203-210, pl. 1, figs. 1-4; alga, closely resembles Agardhiella tenera; Lower Cretaceous; Aude, Ariége, Basses-Pyrénées, southern France, and Navarre province, Spain.

AGROSTISTACHYOPHYLLUM Rásky, (1965) 1966.

Agrostistachyophyllum tomharrisi Rásky, (1965) 1966, p. 264, pl. 1, fig. 1; leaves, Euphorbiaceae; upper Eocene; Budapest-Obuda, Hungary.

ALANGIOXYLON Awasthi, (1968) 1969.

Alangioxylon scariforme Awasthi (1968) 1969 , p. 322-325; figs. 13 ; pl. 1, figs. 1, 3-6; wood, Alangiaceae; middle Tertiary; northnorthwest Pondicherry, Arcot district, Madras State, India.

ALATOCARPUS Lele (1968) 1969. Alatocarpus indicus Lele, (1968) 1969. p. 52-55, figs. $1,2,7-10$; pl. 1 , figs. $1-5,12-15$, a platyspermic seed; Permian; Singrauli coalfield, Uttar Pradesh, India.

ALBERTLONGIA Taylor, 1967.

Albertlongia incostata Taylor, 1967, p. 26-28, figs. 1, 2, pl. 9, figs. 1017; pteridosverm seed; Middle Pennsylvanian; Carrier Mills, Illinois, U.S.A.

ALBERTAPORELLA Johnson, 1966. Albertaporella involuta Johnson, 1966 , p. 1386 , pl. 176 , figs. 2-8; green algae, Dasycladaceae; Mississippian; along South Berland River, north of Jasper National Park, Alberta, Canada.

ALCHORNEAEPHYLLUM Rásky, (1965) 1966.

Alchorneaephyllum chandleri Rásky, (1965) 1966, p. 264-265, pl. 1, fig. 3; leaves, Euphorbiaceae; upper Eocene; Budapest-Obuda, Hungary.

ALDANELLA Kolosov, 1966.

Aldanella monstrata Kolosov, 1966 ,

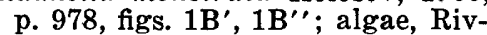
ulariaceae; Precambrian; Olekma river basin, Yakut SSR.

ALDANIA Krylov, 1969.

Aldania sibirica (Jackolev, 1934) Krylov, in Krylov, Korolyuk and Siderov, 1969 , p. 200-203, figs. 50, 51 ; pl. 37, figs. $3-5$; stromatolite; Precambrian; Aldan river basin,
Siberian platform, northeastern Siberia, U.S.S.R.

ALSTAETTIA Remy and Remy, 1969. Alstaettia andersoni Remy and Remy, 1969, p. 91-129, figs. 1-7; pls. 20-23; stem, Matoniceae; middle Barremian; Alstaettia, Ahaus district, North Rhine-Westphalia.

ALTERNELLA Raaben, 1972.

Alternella hyperboerica Raaben, 1972 , p. $47-49$, fig. 10 ; pl. 29 , figs. 1-4; stromatolite; lowermost Cambrian; Severnaya Zemlya and Spitsibergen.

ALTERNIA Vologdin, 1962.

Alternia silva Vologdin, 1962, pt. 2, p. 519-521, fig. 29; pl. 25, figs. 1-4; stromatolite, Trichostromaceae; Lower Ordovician; right bank of the Angara near Boguchany village, Krasnoyarskiy Kray, U.S.S.R.

ALTERNICUTIS Schneider, 1969.

Alternicutis gibba Schneider, 1969, p. 29-30, fig. 8; pl, 9, figs. 5-7; cuticle, Glumiflorae; middle Miocene; Kolenfeld Nochten, Lower Lusatia, east-central Germany.

ALTOCHARA Saydakovskiy, 1968.

Altochara continua Saydakovskiv, 1968 , p. 103-104, pl. 15, figs. 22 , 23; charophyte, fruit; Caspian depression, U.S.S.R.

AMADOCARPUS Novik, 1968.

Amadocarpus beschevensis Novik, 1968 , p. $199-200$, pl. 51, figs. 17 , 18; seed, incertae sedis; Lower Carboniferous; western outskirts of the Donets basin, U.S.S.R.

AMADOCOPTERIS Zalessky, 1944. Amadocopteris rossica Zalessky, 1944 , p. 190-192, fig. 3; fernlike leaf, Marattiaceae; Permian and Carboniferous; Donets basin, U.S.S.R.

AMGAINA Korde, 1973.

Amgaina compacta Korde, 1973, p. 158-159, pl. 30, figs. 1, 2; algae, Parachabkoviaceae; Middle Cambrian; Amga river, Yakutsk, U.S.S.R.

AMORFIA Rácz, (1964) 1966.

Amorfia jalinki Rácz, (1964) 1966, p. $85-86$, pl. 8 , figs. $1-5$; calcareous, red algae of uncertain affinities; the San Emiliano and Lois-Ciguera Formations, Carboniferous; Leon province, northwestern Spain.

ANDIROXYLON Müller-Stoll and Mädel. 1967.

Andiroxylon biseriatum Müller-Stoll and Mädel, 1967, p. 126-130, figs. 
$7 \mathrm{a}, \mathrm{b}$; pl. 31 , figs. 44,$45 ;$ pl. 32 , figs. $48-50$; pl. 33 , figs. $53-56$; wood, Leguminosae; Tertiary; Kenya, east Africa.

ANGAROPHLOIOS Meyen, 1972.

Angariphloios leclercqianus Meyen, 1972 , p. 154-156, fig. 5 ; pl. 1, figs. 7-10; bark, lepidophyte; Carboniferous; Angaraland, Siberia, U.S.S.R.

ANGAROPHYCUS Vologdin, 1962.

Angarophycus depictus Vologdin, 1962 , pt. 1 , p. $177-180$, figs. 13 , 14 ; pl. 10, figs. 2,3 ; pl. 11, figs. 1,2 ; stromatolite, Lermontvaephycaceae; Sinian; right bank of the Angara river above Kamenka village, U.S.S.R.

ANGARORADIX Khakhlov, 1964.

Angaroradix bellis Khakhlov, 1964, p. 55-56, pl. 1, figs. 2, 3; Equisetales; upper Paleozoic; northern Siberia.

ANGIOPORELLA Masse, Conrad and Radoičić, 1973.

Angioporella fouryae Masse, Conrad, and Radoičić, 1973, p. 383387 , fig. 1 ; pl. 1 , figs. $1-8$, calcareous alga, Dasycladaceae; Lower Cretaceous ; Bouches-du-Rhône, southeastern France.

ANGULOCELLULARIA Vologdin, 1962.

Angulocellularia anistoma Vologdin, 1962 , pt. 2, p. $484-485$, fig. 5; algae, Sajaniaceae; Lower and Middle Cambrian; Yanguda river basin, U.S.S.R.

ANSOPORELLA Gnilovskaya, 1972. Ansoporella ansa Gnilovskaya, 1972, p. 116-119, fig. 51 ; pl. 15, fig. 2 ; alga, Moniliporellaceae; Middle and Upper Ordovician; eastern Kazakhstan, U.S.S.R.

ANTIQUOPHYTOLITHUS Vologdin, 1962.

Antiquophytolithus filimentaris Vologdin, 1962, pt. 1, p. 262-264, fig. 58 , pl. 54, figs. 1-4; stromatolite, Trichostromataceae; lower Sinian, right bank of the Angara river, Grebensky Byk, eastern slope of the Yenisey ridge, U.S.S.R.

AOUJGALIA Termier and Termier, 1950.

Aoujgalia variabilis Termier and Termier, 1950, in Petryk and Mamet, 1972, p. 791, 793, pl. 9, figs. $1-5$; calcareous algae (originally described by Termier and Termier as a foraminifer): Lower Carboniferous; central Morocco and southwestern Alberta, Canada.
APHRODITICODIUM Elliott, 1970.

Aphroditicodium aurantium Elliott, 1970 b, p. $329-330$, pl. 62 , figs. 1, 2; algae, Codiaceae; Permian, Ora Mosul, northern.Iraq, Middle East.

APHROPORELLA Gnilovskaya, 1972. Aphroporella gracilis Gnilovskaya, 1972 , p. $72-76$, figs. $32-34$, pl. 4 , fig. 4 ; pl. 5 , figs. $2-4$; alga, Dasycladaceae; Upper Ordovician; eastern Kazakhstan, U.S.S.R.

APOROTHALLUS Krasilov, 1973.

Aporothallus ladyzhenskajae Krasilov, 1973 , p. $99-100$, pl. 46 , figs. 58-64; pl. 47, figs. $65-74$; bryophyte, Hepaticae; Upper Jurassic; Bureya basin, near the mouth of the Umalta river, U.S.S.R.

APTEROCLADUS Archangelsky, 1966.

Apterocladus lanceolatus Archangelsky, 1966 , p. 282-286, figs. 27-28, 34 ; pl. 6, figs. 40-48; pl. 7, figs. 52,55 ; pl. 8 , figs. $68-70$; gymnospermous cones and leafy twigs, Podocarpaceae; Lower Cretaceous; Santa Cruz province, Argentina.

ARCHAEOCYCAS Mamay, 1973.

Archaeocycas whitei Mamay, 1973 , p. 687-689, fig. 1a-c; fertile organ, Cycadales; Lower Permian; Baylor County, Texas, U.S.A.

ARCHAEOLARIX Teslenko, 1970.

Archaeolarix argunensis Teslenko, 1970a, p. 102-104, pl. 13, figs. 1-6; twigs and small female cones, Pinaceae; Lower Cretaceous; left bank of Argun river, Pavlovka village near settlement of Nerchinskiy Zavod, Transbaikalia, southeastern Siberia, U.S.S.R.

ARCHAEOSPERMA Pettitt and Beck, 1968.

Archaeosperma arnoldii Pettitt and Beck, 1968, p. 140-153; figs. 1-3; pls. 1-5; cupulate seed, Gymnospermae; Upper Devonian; northern Pennsylvania, U.S.A.

ARCTOPHYTON Schweitzer, 1968.

Arctophyton gracile Schweitzer, 1968 , p. $49-55$, figs. 2-4; pl. 6, figs. $2-4$; pl. 7, figs. $1,3-6 ;$ pl. 8 , figs. $1-4$; pl. 9, figs. 1-5; Progymospermopsida, compared with Aneurophyton, Calamophyton, and Hyenia; Devonian; northern Vestspitsbergen.

ARENARINA Vologdin, 1962.

Arenarina columnella Vologdin, 1962 , pt. 2, p. 505-508, fig. 20; pl. 17, figs. 1-3; stromatolite, Trichostromaceae: Ordovician; area of the Angara above Boguchany 
village, Krasnoyarskiy Kray, U.S.S.R.

ASCIDIELLA Grambast, 1966.

Ascidiella iberica Grambast, 1966 , p. 2210 , pl. 1, figs. 1-6; charophyte, Clavatoraceae; Lower Cretaceous; Tarragona province, Spain.

ASPHALTINA Mamet, 1972.

Asphaltina cordillerensis Mamet, in Petryk and Mamet, 1972, p. 795, 797 , fig. 8 ; pl. 10, figs. $3-6$; algae, South Misty Range, section 20, Mount Head Formation; Lower Carboniferous; southwestern Alberta, Canada.

ASTRALOCAULIS Hueber, 1971.

Astralocaulis davidii (Haris, 1929) Hueber, 1971a., p. 640-641; a new name for Schizopodium Harris; axes, incertae sedis; Devonian; Burdekin basin, north Queensland, Australia.

ASTRALOPTERIS Tidwell, Rushforth and Reveal, 1967.

Astralopteris coloradoca (Brown, 1950) Tidwell, Rushforth and Reveal, 1967, p. 237-240, 1 fig., pls. 1-6; fronds, clasely related to Drynaria; Polypodiaceae; Cretaceous; Utah and Colorado, U.S.A.

AURICULOPORA Bock, 1969.

Auriculopora acrostichioides Bock, 1969 , p. 101-106, figs. 164-167; ? fern, Marattiales; Triassic; Winterpock, Virginia, U.S.A.

AUSTRALOXYLON Marguerier, 1973.

Australoxylon teixeirae Marguerier, 1973 , p. 39-43, figs. $1-4$; pl. 1 , figs. $1-5$; pl. 2 , figs. $1-4$; pl. 3 , figs. 1, 2 and 4; wood, Gymnospermae; Permian; Tete basin, $21 \mathrm{~km}$ east of Carinde, Mozambique.

AUSTROSTROBUS Morbelli and Petriella, 1973.

Austrostrobus ornatum Morbelli and Petriella, 1973, p. 279-288, 1 fig., pls. 1, 2; lycopsid cone; Triassic Santa Cruz province, Argentina.

AVELINESIA Stockmans, 1968.

Avelinesia antiqua (Dawson, 1871) Stockmans, 1968, p. 33-34, pl. 4, fig. 7 ; pl. 9, fig. 6 ; incertae sedis; Middle Devonian; Brabant Massif region, central Belgium.

AXOTHRIX Nagy, (1969) 1971.

Axothrix malmica Nagy, (1969) 1971 , p. 306-307, 315-316, pl. 1, fig. 7; algae, incertae sedis; Upper Jurassic; Mecsek mountains, Hungary.
AZYRTALIA Volodgin and Drosdova, 1969.

Azyrtalia zonulata Vologdin and Drosdova, 1969a, p. 1162-1163, figs. I-1, 4 ; algae, Rivulariaceae; upper Precambrian; Batenev ridge, Krasnoyarsk, central Siberia, U.S.S.R.

\section{B}

BACANELLA Pantíc, 1971.

Bacanella floriformis Pantíc, 1971. p. 105-108, 110-111, fig. 2; pls. 1-3; alga, Codiaceae?; Middie Jurassic; left bank of the Piva river, Montenegro, southern Yugoslavia.

BAIEROPHYLLITES Jain and Delevoryas, 1967.

Baierophyllites florinii Jain and Delevoryas, 1967 , p. 577-578, pl. 95 , figs. 1-5; pl. 96, figs. 1-3; cuticle and leaf compressions, Ginkgoaceae; Middle Triassic; Minas de Petroleo, southwest of Mendoza, western Argentina.

BALIOSPERMOPHYLLUM Rásky, (1965) 1966.

Baliospermophyllum kraeuseli Rásky, (1965) 1966, p. 266, pl. 2, figs. 4, 5; leaves, Euphorbiaceae; upper Eocene; Budapest-Obuda, Hungary.

BAMBAKIA Anisimova, 1973.

Bambakia cyclopteroides. Anisimova, 1973a, p. 140-141, 1 fig., fernlike foliage, Middle Carboniferous; Bambak ridge, North Caucasus, U.S.S.R.

$B A P H I O X Y L O N$ Lakhnapal and Prakash, 1970.

Baphioxylon dechampsii Lakhnapal and Prakash, 1970, p. 15-17, pl. 10 , figs. $33-36$; pl. 11 , figs. 37,39 ; wood, Leguminosae; Miocene; Lake Albert, Congo, east-central Africa.

BAQUEROITES Herbst, 1966.

Baqueroites padulae Herbst, 1966, p. 83-85, figs. 5-7; pl. 2, figs. 4-6; foliage, probably a "pteroid" fern; Lower Cretaceous; Santa Cruz, Patagonia, Argentina.

$B A S I S P H A E R A$ Walter, 1972.

Basisphaera irregularis Walter, 1972 , p. 132-136, figs. $6,24,37-$ 38 ; pl. 2 , fig. 1 ; pl. 18 , figs. 3,4 ; pl. 19, figs. $1,3-5$; stromatolite; upper Riphean (Adelaidean); Amadeus basin, Australia.

BAVARICUTIS Roselt and Schneider. 1969.

Bavaricutis angustiora Roselt and Schneider, 1969 , p. 82, textfig. 32, 
pl. 20, fig. 2 ; cuticle, ?Ginkgoales; Jurassic; Theta near Bayreuth, Bavaria, Germany.

BEHUNINIA Chandler, 1966.

Behuninia joannei Chandler, 1966, p. 145-149, figs. 1-12; pls. 1-4, figs. 1-43; seeds, Cycadales; Upper Jurassic, Utah, U.S.A.

BENLIGHTFOOTIA Lacey and Huard-Moine, 1966.

Benlightfootia mackii Lacey and Huard-Moine, 1966, p. 20, 21, fig. 2; bifid leaves, incertae sedis; Lower Permian; Wankie beds, southern Rhodesia.

BERCHEMIOPSIS MacGinitie, 1969.

Berchemiopsis paucidentata MacGinitie, 1969, p. 120-121, pl. 13, fig. 5; leaf, Rhamnaceae; Eocene; Wardell ranch, Rangely, Colorado, U.S.A.

BERLINIOXYLON Müller-Stoll and Mädel, 1967.

Berlinioxylon quirogae (Schenck) Müller-Stoll and Mädel, 1967, p. 108-111, fig. 3a, b; pl. 26, figs. 9-11; pl. 27, figs. 13-16; wood, Leguminosae; Pliocene; Spanish West Sahara.

BIGNONIACE AESPERMUM Straus, 1969.

Bignoniaceaespermum germanicum Straus, 1969 , p. 187 , pl. 29, fig. 22; seed, Bigoniaceae; Pliocene; Willershausen, Germany.

BORISIELLA Khakhlov, 1964.

Borisiella bella Khakhlov, 1964, p. $83-84$, pl. 32, fig. 127; pl. 35, fig. 137; Equisetales; upper Paleozoic, northern Siberia.

BORLOGELLA Vologdin, 1962.

Borlogella multigaria Vologdin, 1962 , pt. 1, p. 270-271, fig. 59; pl. 58. figs. 1-5; stromatolite, Sarmallaceae; upper Sinian; Borlog river of the Burul'deyka river system. southwestern Cisbaikal, U.S.S.R.

BRACHYSTEGIOXYLON Lakhnapal and Prakash, 1970.

Brachystegioxylon premicrophyllum Lakhnapal and Prakash, 1970, p. 13-15, pl. 8, figs. 26-28; pl. 9, figs. 29,31 ; wood, Leguminosae; Miocene; Lake Albert, Congo, east-central Africa.

BRASENIELLA Dorofeev, 1973.

Braseniella nymphaeoides (Dorofeev, 1958) Dorofeev, 1973, p. 110-111, pl. 12, figs. $1-3$; seed, similar to Nymphaea; Oligocene; right bank of the Tavdy river. Sverdlovskaya and Tyumenskaya Oblast, U.S.S.R.
BRAUNIA Givulescu, 1970.

Braunia tiliaefolia (A. Braun, 1845) Givulescu, 1970, p. 242, pl. 1 , figs. $1-4 ;$ pl. 2 , figs. $1-4 ;$ pl. 3 , figs. $1-3$; epidermis, incertae sedis; Sarmatian; Oehningen, Germany.

BULBISTROMA Vologdin, 1962.

Bulbistroma curtotthallum Vologdin, 1962 , pt. 1 , p. 300-302, fig. 80 ; algae, Scandophycaceae; middle Sinian; right bank of the Nizhney Tunguska, about $11 \mathrm{~km}$ from the mouth, Turukhansk region, U.S.S.R.

BUREJOSPERMUM Krasilov, 1972.

Burejospermum crassitestum Krasilov, 1972 , p. $65-66$, pl. 22 , figs. 14, 9-14; ginkgophyte seed, Pseudotorelliaceae; Upper Jurassic to Lower Cretaceous; Bureya river basin, Khabarovsk Territory, U.S.S.R.

BURSIPHYCAS Vologdin, 1962.

Bursiphycas bullatus Vologdin, 1962 , pt. 1, p. 230-231, fig. 39, pl. 40 , fig. 2, lower part of fig. 3 ; stromatolite, Vesiculariaceae: Lower Cambrian; Borlog river, southwest of Cisbaikal, U.S.S.R.

BUTEOXYLON Barnard and Long, 1973.

Buteoxylon gordonianum Barnard and Long, 1973, p. 91-99, figs. 14 ; pl. 1, figs. $1-9$; pl. 2 , figs. 10 20 ; pl. 3, figs. $22-29$; a petrified stem, Buteoxylonaceae; upper Carboniferous; Oxroad Bay, East Lothian, Scotland.

\section{C}

CAIROA Matten, 1973.

Cairoa lamanekii Matten, 1973, p. $621,625,627$, figs. $14-35$; axes and leaves, Progymnospermopsida; Middle Devonian; near Cairo, Green County, New York, U.S.A. CALKINSIA Wolfe, 1968.

Calkinsia franklinensis Wolfe, 1968, p. 20, pl. 4, figs. 4, 8; leaves, Menispermaceae; lower Tertiary; King County, Washington, U.S.A.

CALLANDRIUM Stidd and Hall, 1970.

Callandrium callistophytoides Stidd and Hall, 1970, p. 398-402, figs. 1-24, the probable pollen-bearing organ of the seed fern Callistophyton; Pennsylvanian; Lawrence County, Illinois, U.S.A.

CAMBRINA Korde, 1973.

Cambrina fruticulosa Korde, 1973, p. 133-134, fig. 18; pl. 15, fig. 1; algae, Cambrinaceae; Lower Cam- 
brian; Bazaikha river, eastern Sayan, U.S.S.R.

CANIPA Skog, 1969.

Canipa quadrifida Skog, in Skog, Andrews, and Mamay, 1969, p. 281-286, figs. 1-9; synangial fructification, pteridosperm; Middle Pennsylvanian; Fayetteville County, West Virginia, U.S.A.

CAREYOXYLON Awasthi, (1969) 1970.

Careyoxylon pondicherriense Awasthi, (1969) 1970, p. 70,73, figs. $6-10$; pl. 2, figs. 6,8 ; wood, Lecythidaceae; Tertiary; near Pondicherry, southern India.

CANNAITES Trivedi and Verma, 1971.

Cannaites intertrappea Trivedi and Verma, 1971, p. 174-185, figs. 1-4; pls. 29, 30, figs. 1-12; pseudostem and root, Cannaceae; Eocene; Mohgaonkalen, Chhindwara district, Madhya Pradesh, India.

CARINOSTROBUS Baxter, 1971.

Carinostrobus foresmani Baxter, 1971 , p. $125-128$, figs. 1,2 ; pls. 33-36, figs. 1-17; cone, Lycophyta; Middle Pennsylvanian; north of Hallowell, Kansas, U.S.A.

CARPATHOPORELLA Dragastan, 1967.

Carpathoporella occidentalis Dragastan, 1967, p. 444-446, pl. 1, figs. $7-9$; calcareous algae; Upper Jurassic, and Lower Cretaceous; Mount Apuseni, Rumania.

CATANGOPHYTON Malich, 1966.

Catangophyton antiquum Malich, 1966 , p. 1207-1209, fig. $1 \mathrm{a}-\mathrm{b}$; shoots, incertae sedis; Upper(?) Cambrian; basin of the Velmo river, left tributary of the Podkamennaya Tunguska, western part of the Siberian platform, U.S.S.R.

CATENOPTERIS Phillips and Andrews, 1966.

Catenopteris simplex Phillips and Andrews, 1966 , p. 122-123, figs. 1-13; fernlike stem, pteridophyte; Upper Carboniferous; Lawrence County, Illinois, U.S.A.

CATINELLA Pflug, 1966.

Catinella polymorpha Pflug, 1966, p. $65-66$, pl. 27 , figs. $10-15,17-$ $68,70-74$; pl. 28 , figs. 4, 5: ?algae, ?Cyanophyta; Precambrian: Idaho-Montana, U.S.A.

CAUCASIA Anisimova, 1973.

Caucasia ginkgophylloides Anisimova, $1973 \mathrm{~b}$, p. 138-139, 2 figs., fronds with leaves, Ginkgoales:
Middle Carboniferous; north Caucasus, U.S.S.R.

CAUDOMORPHA Vologdin, 1962.

Caudomorpha cataphracta Vologdin, 1962 , pt. 2, fig. 19 ; pl. 16, figs. 1-4; microscopic algae; Lower Cambrian; region of the Angara above the mouth of the Agaleevo river, U.S.S.R.

CERCIDIPHYLLOXYLON Prakash, Březinová and Bưžek, 1971.

Cercidiphylloxylon kadanense Prakash, Březinová and Bủžek, 1971, p. $110-112$, pl. 36 , fig. 34 ; pl. 37 , figs. 35, 36; gymnospermous wood, Cercidiphyllaceae; Oligocene; Doupovské Hory mountains, northern Bohemia, Czechoslovakia.

CHAPELIA Beck and Bailey, 1967.

Chapelia campbellii Beck and Bailey, 1967, p. 998-007, figs. 127; ?pteridosperm petiole; Upper Devonian or Lower Mississippian; near Jacob's Chapel, north of New Albany, Indiana, U.S.A.

CHEIRORHIZA Krasilov, 1970.

Cheirorhiza brittae Krasilov, 1970, p. 132-141, figs. 1-3; pl. 11, figs. $4-8$; pl. 12, figs. 1-10; leafy liverwort, Jungermanniales; Upper Jurassic; right bank of the Bureya river; Khabarovsk Territory, eastern Siberia, U.S.S.R.

CHLIDANOPHYTON Gensel, 1973.

Chlidanophyton dublinensis Gensel, 1973 , p. 145, figs. $1-6$; pls. $33-40$, figs. 1-22; plants with erect main axes bearing spirally arranged branch systems; incertae sedis (? Coenopteridopsida or ? Progymnospermopsida); Lower Mississippian; Pulaski County, Virginia, U.S.A.

CHLUPACIA Obrhel, 1969.

Chlupacia moravica Obrhel, 1969, p. 56-57, pl. 1, fig. 4; pl. 2, figs. $1-4$; pl. 3, figs. 1,2 ; woody axes, incertae sedis; Middle Devonian; Chabičov, Moravia, Czechoslovakia.

CHOMENTOVSKIA Vologdin, 1962.

Chomentovskia vesiculosa Vologdin, 1962 , pt. 2, p. 516-518, figs. 27 , $28 \mathrm{a}, \mathrm{b}$; pl. 22 , fig. 3 ; pl. 24 , figs. 1-3; stromatolite, Trichostromaceae; Ordovician; left bank of the Angara about $4 \mathrm{~km}$ above Boguchany village, Krasnoyarskiy Kray, U.S.S.R.

CHOMUSTACHIA Korde, 1973.

Chomustachia tuberosa (=Chabakovia tuberosa Korde, 1961) Korde, 1973, p. 156-157, pl. 29, fig. 2; algae, Parachabakovi- 
aceae; Lower Cambrian; Kiya river, Kuznetskiy Alatau, Amga river, Yakutsk, U.S.S.R.

CIRCONITELLA Watson, 1969.

Circonitella knowltoni (Seward) Watson, 1969 , p. 214-217, figs. 26 ; pl. 1, figs. $1-5$; fruit, thallophyte; Wealden; England.

CIRRIPHYCUS Vologdin, 1962.

Cirriphycus ordinatus Vologdin, 1962 , pt. 1 , p. 244-247, figs. 47 , 48; pl. 46, fig. 1 ; stromatolite, Telastromataceae; Sinian; lower Nizhney Tunguska, right bank, above Durnoy cape, Turukhansk area, U.S.S.R.

CODIAEPHYLLUM Rásky, (1965) 1966.

Codiaeophyllum palaeovarigatum Rásky, (1965) 1966, p. 266, pl. 3, figs. 9-11; leaves, Euphorbiaceae; upper Eocene; Budapest-Obuda, Hungary.

COLCHIDIA Kolakovskii and Schakryl, 1968.

Colchidia angustissima Kolakovskii and Schakryl, 1968, p. 67-69, pl. 6, figs. 1, 2; needle petrifactions and impressions, compared with Cathaya, Pinaceae; Miocene, Sarmatian; northern foot of the Kavaluk upland near Barmysh and Mugudzyrkhva villages, Abkhaz, U.S.S.R.

COLUMNARIA Vologdin, 1962.

Columnaria turuchanica Vologdin, 1962 , pt. 1 , p. 235-238, fig. 44; pl. 43, figs. $1-3$; stromatolite, Vesiculariaceae; Sinian; right bank of the Yenisey above Miroedikh village, Turukhansk region, U.S.S.R.

COMPSOSTROBUS Delevoryas and Hope, 1973.

Compsostrobus neotericus Delevoryas and Hope, 1973, p. 815-816, figs. $1-12,14,15,17,26,27$; ovulate conifer cone, Compsostribaceae; Upper Triassic; Deep River basin, North Carolina, U.S.A.

CONCARYON Loubiere, 1956.

Concaryon renaulti Loubiere, 1956 , p. 492-494, figs. 1-3, seed, Pteridospermes-Mesocaryales; Up per Carboniferous; La Grand'Croix, south-central France.

CONCRESCERARIA Vologdin, 1962.

Concresceraria subrotunda 'Vologdin, 1962, pt. 2, p. 540-542, fig. 35; pl. 48, figs. 1-3; algae, Actinophyceae; Lower Cambrian; right bank of the Angara before the mouth of the Agraleevo river, U.S.S.R.
CONIPORELLA Fischer and Thierry, 1971.

Coniporella clavaeformis (d'Archaic, 1843) Fischer and Thierry, 1971, p. 28-31, figs. 2a-b, 4a-c, 5 ; algae, Dasycladaceae; Middle Jurassic, Aisne, France.

CONNEXIA Kochansky Devidé, 1970.

Connexia fragilis KochanskyDevidé, 1970, p. 19-21, pl. 4, fig. 1 ; pl. 6, fig. 5 ; pl. 11, figs. 1-14; pl. 12, figs. 1-4; pl. 15, fig. 1; alga, Dasycladaceae; Middle Carboniferous; near Ricice village, southern Croatia, Velebit mountains, Yugoslavia.

COPAIFEROXYLON Müller-Stoll and Mädel, 1967.

Copaiferoxylon migiurtinum (Chaiarugi) Müller-Stoll and Mädel, 1967 , p. 133-134; wood, Leguminosae; Miocene; Somaliland.

CORBULARIA Vologdin, 1962.

Corbularia conglutinata Vologdin, 1962 , pt. 2 , p. 538-540, fig. 34a, b; pl. 32, figs. 1-3; stromatolite, Actinophyceae; Lower Cambrian; area of Irkineevo, right tributary of the Angara, slightly below the mouth of the Chugumey, Krasnoyarskiy Kray, U.S.S.R.

CORNUTULA Korde, 1973.

Cornutula kaltatica Korde, 1973, p. 122-123, pl. 8, fig. 2; algae, Cornutulaceae; Lower Cambrian; Bazaikha river, eastern Sayan, U.S.S.R.

CORONICUTIS Roselt and Schneider, 1969.

Coronicutis hartauensis Roselt and Schneider, 1969, p. 77-78, fig. 27; pl. 19 , figs. 3,4 ; cuticle of undetermined dicotlyedon; lower Miocene; Hartau near Zittau, East Germany.

CORYLOCARPINUS Straus, 1969.

Corylocarpinus szaferowae Straus, 1969 , p. $173-174$; pl. 30 , figs. $49 \mathrm{a}$, $49 \mathrm{~b}$; angiospermous fruit; Pliocene; Willershausen, Germany.

CORYLOXYLON Prakash, Březinová and Bůžek, 1971.

Coryloxylon nemejcii Prakash, Březinová and Bưžek, 1971, p. 116-118, pl. 40, figs. 48-52; wood, Betulaceae; Oligocene; Doupovské hory mountains, northern Bohemia, Czechoslovakia.

CORYPHIOCARPUS Koch, 1972.

Coryphoicarpus globoides Koch, 1972 a, p. $23-28$, fig. 10 ; pl. 4, figs. $8 \mathrm{~A}-\mathrm{B}$; pl. 16 , figs. $1 \mathrm{~A}-\mathrm{C}$; pl. 18 , figs. 1-3; seed, Coryphae; Upper Cretaceous ; Nûgssuaq, West Greenland. 
CORYPHOIDES Koch, 1972.

Coryphoides poulseni Koch 1972b, p. 7-27, figs. $2-8$; pls. $1-13$, and 17, fig. 2; fruit, Palmae; Upper Cretaceous: Nûgssuaq, West Greenland.

COSTICUTIS Roselt and Schneider, 1969.

Costicutis parvicellareata Roselt and Schneider, 1969 , p. 65, pl. 10, fig. 1 ; cuticle of the upper side of angiospermous leaves; middle Miocene; Lower Lusatia, East Germany.

CONTEXTA Gnilovskaya, 1972.

Contexta binata Gnilovskaya, 1972, p. $108-110$, fig. 47 , pl. 12, fig. 2; alga, Moniliporellaceae; Middle and Upper Ordovician; eastern Kazakhstan, U.S.S.R.

CRASSOFILICITES Bock, 1969.

Crassofilicites wherryi Bock, 1969 , p. 162-167, figs. 252-264; taeniopterid foliage; Triassic; Carversville, Pennsylvania, U.S.A.

CRENATICAULIS Banks and Davis, 1969.

Crenaticaulis cerruculosus Banks and Davis, 1969 , p. 444-445, figs. 1-21, 23-31, 34-35; pteridophyte; Lower Devonian; north shore of Gaspé Bay, Gaspé Peninsula, Quebec, Canada.

CRETACICRUSTA Elliott, 1972.

Cretacicrusta dubiosa Elliott, 1972a, p. 501-503, pl. 100, figs. 1-4; pl. 101 , figs. 1,2 ; ?algae, Rhodophyta; Lower Cretaceous; Farrington, England.

CRINITELLA Gnilovskaya,.1972.

Crinitella radiata Gnilovskaya, 1972, p. 134-136, fig. 36 ; pl. 15, fig. 4; alga, incertae sedis; Upper Ordovician; eastern Kazakhstan, U.S.S.R.

CRISPOPHYCUS Vologdin, 1962.

Cryspophycus sibiricus Vologdin, 1962 , pt. 1 , p. $302-303$, fig. 81 ; algae, Scandophycaceae; middle Sinian; right bank of the Nizhney Tunguska, about $11 \mathrm{~km}$ from the mouth, Turukhansk region, U.S.S.R.

CROSSOPTERIS Tidwell, 1967.

Crossopteris utahensis Tidweli, 1967, p. 45-48, figs. 4A, 5A-D; pl. 3, fig. 7 ; pl. 6 , fig. 1 ; pl. 10 , fig. 8 ; pinnules; Lower Pennsylvanian; Utah, U.S.A.

CRUSTOPHYCUS Vologdin, 1962.

Crustophycus angaricus Vologdin, 1962 , pt. 1, p. 195-199, figs. 22, 23 , pl. 20 , figs. $1-7$; pl. 21, figs. 1-3; stromatolite, Crustophycaceae; lower Sinian; right bank of the Angara river beyond $\mathrm{Ka}$ menka village, Krasnoyarskiy Kray, central Siberia, U.S.S.R.

CUPRESSOCONUS Kilpper, 1968.

Cupressoconus thompsoni (Brown MS in Schloemer-Jäger, 1960) Kilpper, 1968, p. 165-166; gymnospermous cones; Tertiary; Germany.

CYANOSTROMA Vologdin, 1962.

Cyanostroma turuchanicum Vologdin, 1962, pt. 1 , p. 287-292, figs. $71-74$; pl. 68 , figs. 1,2 , pl. 69 , fig. 1 ; pl. 70, figs. 1, 2; stromatolite, Plexostromataceae; middle Sinian; Nizhney Tunguska, Durnogo region, U.S.S.R.

CYATHOFORMA Bock, 1969.

Cyathoforma carolinensis (Emmons) Bock, 1969, p. 119-127, figs. 190-100 ; for Pecopteris carolinensis Emmons, 1856; fern, Cyatheaceae; Triassic; Winterpock, Virginia, U.S.A.

CYCADENIA Bock, 1969.

Cycadenia elongata Bock, 1969, p. 208-213, figs. 329-337; ?cycadalean trunks; Triassic; Gwynedd, Pennsylvania, U.S.A.

CYCULARIA Vologdin, 1969.

Cycularia orbiculata Vologdin, 1969, p. 1379, fig. 4; blue-green algae, Shanganellaceae; Lower Cambrian; Ulug-Shangan canyon, Tuva, southern Siberia, U.S.S.R.

CYNEPTERIS Ash, 1969.

Cynepteris lasiophora Ash, 1969, p. D31-D38, figs. 15,16 ; pl. 2, figs. $1-5$; pl. 3 , figs. $1-7$; pinnate fern leaf, Cynepteridaceae; Upper Triassic; Fort Wingate area, New Mexico, U.S.A.

CYSTOSTROMA Vologdin, 1962.

Cystostroma varians Vologdin, 1962 , pt. 1 , p. $276-278$, figs. 65 ; pl. 63 , figs. 1-4; stromatolite, Sarmallaceae; upper Sinian; Burul'deyka river, western Cisbaikal, U.S.S.R.

\section{D}

DACRYDIOXYLON Greguss, 1967.

Dacrydioxylon estherae Greguss, 1967, p. 34-36, pl. 22, figs. 1-14; gymnospermous wood, Podocarpaceae; lower Oligocene; Solymár, Hungary.

DAMMAROXYLON Schultz-Motel, 1966.

Dammaroxylon africanum SchultzMotel, 1966, p. 281-283, fig. 1 ; pl. 38 , figs. 1-6; pl. 39, figs. 7-11; gymnospermous wood, Araucariaceae; Upper Cretaceous; East Pondoland, South Africa. 


\section{DAMUDOXYLON Maheshwari} (1966) 1967.

Damudoxylon waltonii Maheshwari (1966) 1967, p. 247-250, figs. 4-7; pl. 3, figs. 15-19; gymnospermous wood; Upper Permian; West Jamuria colliery, Raniganj coalfield, Bengal, India .

$D A R N E Y A$ Schaarschmidt and Maubeuge, 1969.

Darneya pelata Scharrschmidt and Maubeuge, 1969. p. 377-391, figs. $1-14$; pl. 1 , figs. $1 \mathrm{a}-3 \mathrm{~b}$; pl. 2, figs. $4-13$; pl. 3 , figs. $14 a-16$; male gymnospermous fructification; Lower Triassic; Darney, Vosges Department, eastern France.

DASYCIRRIPHYCAS Vologdin, 1962.

Dasycirriphycas fructiculosus Vologdin, 1962, pt. 2, p. 487-488, fig. 6 ; pl. 7, figs. 2, 3; algae, incertae sedis; Lower Cambrian; region of the upper course of the Lena, Manzurka river valley, Irkutsk Oblast, U.S.S.R.

DECHELLYIA Ash, 1972.

Dechellyia gormani Ash, 1972a, p. 607-613, figs. $6 \mathrm{~A}-\mathrm{C}$; pls. 115-118; leafy shoot and foliage, Coniferales: ?family; Upper Triassic: mouth of Canyon de Chelly, Arizona, U.S.A.

DICHROSTACHYOXYLON MüllerStoll and Mädel, 1967.

Dichrostachyoxylon acaciaeforme Müller-Stoll and Mädel, 1967, p. 138-140, fig. 10 ; pl. 36, figs. 7476 ; wood, Leguminosae; Severin province, Rumania.

DICTYOCLAVATOR Grambast, 1966.

Dictyoclavator fieri (Donze, 1955) Grambast, 1966, p. 2210; charophyte, Clavatoraceae; JurassicCretaceous boundary; Jura, AlpesMaritimes regions, and Provence regions, France.

DIECTOCHARA Musacchio, 1971.

Diectochara andica Musacchio, 1971, p. $24-35$, fig. 4 ; pl. 1 ; figs. $8-10$; pl. 2, figs. $24-29$; pl. 3, figs. $30-38$; charophyte fructification; Lower Cretaceous; Neuquen province, Argentina.

DIGITULARIA Vologdin, 1962.

Digitularia inconcinna Vologdin, 1962 , pt. 2, p. 530-531, 532-533, fig. 33 , pl. 35 , figs. 1-4; algae, Trichostromaceae; Ordovician; area of the Angara river above Boguchany village, Krasnoyarskiy Kray, U.S.S.R.

DIKIMDINELLA Kolosov, 1966.

Dikimdinella privica Kolosov, 1966, p. 979-980, figs. IC', IC"'; algae, Anabaenaceae; Precambrian; Olekma river basin, Yakut SSR.

DILLENIOXYLON Gregus, 1969.

Dillenioxylon mikofalvense Gregus, 1969 , p. 26-27, pl. 11, figs. 1-10; wood, close connection with Dilleniaceae; Sarmatian; Mikofalva, Hungary.

DINOPHYTON Ash, 1970.

Dinophyton spinosus Ash, 1970, p. $650-651$, figs. $2-6$; pls. 122-124; gymnospermous shoots with foliage; Texas, New Mexico, and Arizona, southwestern U.S.A.

DISTICHOPLAX Pia, 1934.

Distichoplax biserialis (Dieterich) Pia, 1934, p. 15-18, figs. 5-8; for Lithothamnium biserialis Dieterich, 1927; calcareous algae, Melobeiseae; Eocene; eastern Iran.

\section{DIVERSIPHYLLUM Bủžek, 1971.}

Diversiphyllum aesculapi (Heer, 1856) Bủžek, 1971, p. 86-89, fig. 13, pl. 41, figs. 1-14; pl. 42, figs. 1-11; leaves, query Convolvulaceae; Tertiary; Petipsy area (north Bohemian basin), Czechoslovakia.

DIVERSOCALLIS Dragastan, 1967.

Diversocallis undulatus Dragastan, 1967, p. $450-451$, pl. 8, figs. 52- 56; calcareous algae; Upper Jurassic and Lower Cretaceous; Mount Apuseni, Rumania.

DIVERSOPORELLA Gnilovskaya, 1972.

Diversoporella cognata Gnilovskaya, 1972, p. 91-93, fig. 41; pl. 9, fig. 5; alga, Codiaceae; Middle and Upper Ordovician; eastern Kazakhstan, U.S.S.R.

DIXOPODOXYLON Fairon-Demaret, 1969.

Dixopodoxylon goense Fairon-Demaret, 1969 , p. 372-386, 18 figs.; fragment of a stemlike axis, incertae sedis; Middle Devonian; Goe, Belgium.

DOLIANITIA Millan, 1967.

Dolianitia opposita Millan, 1967, p. $5-8$, pl. 1, figs. 1-5; pl. 2, fig. 1; glossopteris female fructification; Upper Carboniferous; Santa Catarina State, Brazil.

DOLIPORELLA Gnilovskaya, 1972.

Doliporella binata Gnilovskaya, 1972 , p. $98-100$, fig. 44 ; pl. 8 , fig. 6 ; alga, Codiaceae; Upper Ordovician; eastern Kazakhstan, U.S.S.R.

DUABANGOXYLON Prakash and Awasthi. (1969) 1970.

Duabangoxylon tertiarum Prakash and Awasthi, (1969) 1970, p. 3840 , pls. 5-6, figs. 24-32; silicified wood, compared with modern wood, 
Sonneiatiaceae; Tertiary; near Jaipur, Assam, India.

DUSEMBAYA Dorofeev, 1973.

Dusembaya turgaica (Dorofeev, 1963) Dorofeev, 1973, p. 107-110, pl. 12, figs. 10-15; seed, Cabombaceae; Oligocene; Kazakhstan, U.S.S.R.

DZERGALANELLA Genkina, 1966.

Dzergalanella dzerganensis Genkina, 1966 , p. $54-55$, pl. 5 , figs. $4-8$; foliage, Sorocaulaceae; lower Mesozoic; Issyk-Kul depression, northern Kirghiz, U.S.S.R.

DZHUNGARODENDRON M. I. Radchenko, 1967.

Dzhungarodendron novikae M. I. Radchenko, 1967, p. 38-39, pl. 8, figs. 1-5; lycopod stem; Carboniferous; Dzhungariya, southeastern Kazakhstan.

\section{E}

ECHANINIA Vologdin and Drosdova, 1969.

Echaninia mucosa Vologdin and Drosdova, $1969 \mathrm{~b}$, p. 440-441, figs. I-1-3; alga, Echaniniaceae; Precambrian; Batenev ridge, central Siberia, U.S.S.R.

EDDYA Beck, 1967.

Eddya sullivanensis Beck, 1967, p. 1-20, figs. 1-25; pls. 1-7; leaves and stem, query gymnosperm, incertae sedis; Upper Devonian; Pond Eddy, Sullivan County, New York, U.S.A.

ELEGANOPTERIS Mogucheva, 1969.

Eleganopteris tripannata Mogucheva, in Mogucheva and Il'ina, 1969, $p$. 131-135, pl., figs. 1-8; Filicales, incertae sedis; Lower Triassic; Tunguska syneclise, U.S.S.R.

ELIGODENDRON Archangelsky and Le La Sota, 1966.

Eligodendron braniase Archangelsky and De La Sota, 1966, p. 17-26, fig. 1; pls, 1, 2; lycopod stem; Permian; Copacabana Peninsula, Bolivia.

EMBERGERELLA Grambast, 1969.

Embergerella cruciata Grambast, 1969 , p. 879 , 881, pl. 2 , figs. 8a-14; charophyte, Clavatoraceae; Cretaceous; Alcala Vega, Teruel province, Spain.

EMBERGERIXYLON Lemoigne, 1968.

Embergerixylon alpinum (Y. Lemoigne) Lemoigne, 1968, p. 155156 , pl. 13, figs. 1-5; gymnospermous wood; Upper Jurassic; Hautes-Alpes, France.

ENORMICUTIS Schneider, 1969.

Enormicutis amplicavata Schneider, 1969 , p. $24-25$, pl. 8 , figs. 2,3 ; cuticle, Taxodiaceae; upper Oligocene; Tagebau Holzwiessig near Bitterfeld, central Germany.

ENTSOVIA Meyen, 1969.

Entsovia rarisulcata Meyen, 1969a, p. 93-96, figs. 1-3; pl. 14, figs. 16 ; teniate leaves, incertae sedis; Permian; eastern European part of the U.S.S.R.

EOHOSTIMELLA Schopf, 1966.

Eohostimella heathana Schopf, in Schopf and others, 1966, p. D71D72; fig. 2c-r; erext axes, incertae sedis; Lower Silurian; Aroostock County, Maine, U.S.A.

EOLEPIDOLPHLOIOS M. I. Radchenko, 1967.

Eolepidophloios quadratus M. I. Radchenko, 1967 , p. 39-40, pl. 9, figs, 1-3; lycopod stem; Carboniferous; Dzhungariya, southeastern Kazakhstan, U.S.S.R.

EOLITHOPORELLA Johnson, 1966.

Eolithoporella dawsoni Johnson, 1966 , 1386-1386, pl. 176, figs. 9, 10; red algae, ?Solenoporaceae; Mississippian; Along South Berland River, north of Jasper National Park, Alberta, Canada.

EORHIZA Robison and Person, 1973.

Eorhiza arnoldii Robison and Person, 1973 , p. 1374-1378, pls. 1-4, figs. 1-20; dicotyledonous rhizomes, order and family incertae sedis; Eocene; British Columbia, Canada. EPIPHYTONOIDES Korde, 1973.

Epihytonoides sanashtykgolicus Korde, 1973, p. 193-194, 197-198, pl. 40, figs. 1, 2; algae, Epiphytaceae; Lower Cambrian; Sanashytkgol river, left tributary of Abakan river, western Sayan, U.S.S.R.

EPISTACHEOIDES Petryk and Mamet, 1972.

Epistacheoides nephroformis Petryk and Mamet, 1972, p. 787, 789, 791, fig. 6 ; pl. figs. $1-10$; algae, Etherington Formation, lower Carboniferous; southwestern Alberta, Canada.

EQUISETOPHYTON Schweitzer, 1972.

Equisetophyton praecox Schweitzer, 1972 , p. 170-173, fig. 14 ; pl. 39, figs. 4, 5 ; equisetalean stem; Middle Devonian; Lindlar, Rheinland, West Germany.

ERBINA Korde, 1973.

Erbina aristata Korde, 1973, p. 142 143 , fig. 24 ; pl. 19 , figs. $1-3$; pl. 20, fig. 1; algae, Erbinaceae; Lower Cambrian; Kuznetskiy Alatau, Batenev ridge, and area of Sukhaya Erba village, southeast of Dolgiy Mys mountains, U.S.S.R. 
ERYTHROPHLOEOCOXYLON

Müller-Stoll and Mädel, 1967,

Erythrophloeocoxylon varians (Felix) Müller-Stoll and Mädel, 1967, p. $118-120$, fig. 5 ; pl. 29 , figs. 27 , 28; wood, Leguminosae; Oligocene; West Indies.

ESTHERELLA Boersma and Visscher, 1969.

Estherella gracilis Boersma and Visscher, 1969 , p. 58, figs. 2 , 3 ; pl. 1 , fig. 1 ; pl. 2, fig. 2 ; dichotomous plant, incertae sedis; Upper Permian; southern France.

EUCASPSIPHORA Cloud and Semikhatov, 1969.

Eucaspsiphora paradisa Cloud and Semikhatov, 1969, p. 1039-1040, fig. 8 ; pl. 4, figs. $1-3$; stromatolite; Paradise Creek Formation, Proterozoic, north-Western Queensland, Australia.

EUACICIOXYLON Müller-Stoll and Mädel, 1967.

Euacicioxylon bharadwajii (Navale) Müller-Stoll and Mädel, 1967, p. 137; wood Leguminosae; Tertiary; India.

EUPALAEOSTACHYA Ishchenko, 1965.

Eupalaeostachya devonica Ishchenko, 1965 , p. $48-50$, pl. 17, figs. 1-6; stems with sporangia, Sphenopsida; Middle Devonian; Donets basin, Ukranian SSR.

EXVOTARISELLA Elliott, 1970.

Exvotarisella maponi Elliott, 1970a, p. $446-449$, pl. 82 , figs. $1-5$; pl. 83 , figs. 1-5; algae, Dasycladaceae; Lower Carboniferous; Northumberland, Great Britain.

\section{F}

FALCICUTIS Schneider, 1969.

Falcicutis varians Schneider, 1969 , p. 21 , 22, fig. 3 ; pl. 5, figs. $3-5$; pl 6, figs. 1-6; cuticle, Leguminosae; middle Miocene; Upper Lusatia, east-central Germany.

FAUEROXYLON Koeniguer, 1970.

Faueroxylon princeps Koeniguer, 1970 , p. $143-148$, figs. 1 , 2; paleoxylotomous wood; post-Eocene; Termit massif, Republic of Niger, western Africa.

FAVOPORELLA Sokač, 1968.

Favoporella annulata Sokać, 1968, p. 207-212, fig. 1 ; pls. $1-4$; calcareous algae, Dasycladaceae; Middle Triassic; Velebit mountain, western Croatia, Yugoslavia.

FIBROSTROMA Vologdin, 1962.

Fibrostroma fibrillatum Vologdin, 1962, pt. 1, p. 261-262, fig. 57; pl. 53, figs. 1, 2; stromatolite, Tricho- stromataceae; Sinian; Kurtun river, right tributary of the Burul'deyka river, southwestern Cisbaikal, U.S.S.R.

FILARIA Korde, 1973.

Filaria seriata Korde, 1973, p. 129 130, pl. 12, fig. 1 ; algae, Cambrinaceae; Lower Cambrian; Bazaikha river, eastern Sayan U.S.S.R.

FILIPHYCUS Vologdin, 1962.

Filiphycus longifolius Vologdin, 1962, pt. 2, p. 544-546, fig. 38 ; pl. 44, figs. 1,2 ; pl. 45 , figs. 1 , $2 \mathrm{a}$; stromatolite, Clorophyta; Ordovician; left bank of the Angara about 4 $\mathrm{km}$ above Boguchany village, Krasnoyarskiy Kray, U.S.S.R.

FILLOSTROMA Vologdin, 1962.

Fillostroma moticum Vologdin, 1962 , pt. 1 , p. $307-309$, figs. 85,86 ; pl. 74 , fig. 2 ; stromatolite, Lamellostromataceae; Lower Cambrian; Borlog river, southwestern Cisbaikal, U.S.S.R.

FINIVERSICUTIS Roselt and Schneider, 1969.

Finiversicutis papillosa Roselt and Schneider, 1969 , p. $64-65$, pl. 8 , fig. 4; cuticle of undetermined axis, probably dicotyledonous; middle Miocene; Sedlitz, Lower Lusatia, East Germany.

FISTULELLA Korde, 1973.

Fistulella decipiens Korde, 1973, p. 217-218, fig. 10 , pl. 66, fig. 2, 3; algae, Fistulellaceae; Early Cambrian; Serlig river, eastern TannuOla, Tuva, U.S.S.R.

FLEXIA Vologdin, 1962

Flexia densiuscula Vologdin, 1962, pt. 2, p. 529-530, pl. 33, figs. 1-3; stromatolite, Trichostromaceae; Lower Ordovician; lower Cisangara and left bank of the Angara about $4 \mathrm{~km}$ above Boguchany village, Krasnoyarskiy Kray, U.S.S.R.

FLORINIA Sveshnikova, 1967.

Florinia vilujensis Sveshnikova, 1967, p. 186-187, pl. 12, figs. 9, 10; fragment of coniferous leaf epidermis, Taxaceae; Upper Cretaceous; Vilyui river, western Yakut SSR.

FONINIA Korde, 1973.

Foninia fasciculata Korde, 1973, p. 108-109, pl. 1, fig. 2; algae, Foninaceae; upper Proterozoic; Kuznetskiy Alatau, Batenev ridge, U.S.S.R.

FORTOPTERIS Boersma, 1969.

Fortopteris latifolia (Zeiller, 1878) Boersma, 1969, p. 68-69, figs. 1, 2 ; pls. 1-4; fertile frond, fern; Upper Carboniferous; coal basin of 
the Departments Nord and Pas-deCalais, France.

FURCATOPORELLA Gnilovskaya, 1972.

Furcatoporella coalita Gnilovskaya, 1972 , p. $119-120$, fig. 52 ; pl. 13 , figs. 1-4; alga, Moniliporellaceae; Upper Ordovician; eastern Kazakhstan, U.S.S.R.

\section{G}

GALINIA Vologdin, 1962.

Galinia eximia Vologdin, 1962, pt. 2, p. 521-524, fig. 30 ; pl. 26, figs. $1-3$; pl. 27 , figs. $1-3$; pl. 28 , figs. $1-3$; pl. 32 , fig. 1 ; stromatolite, Trichostromaceae; Lower Ordovician; Boguchany village, Krasnoyarskiy Kray, U.S.S.R.

GEORGIANA Walter, 1972.

Georgiana howchini Walter, 1972, p. 113-115, fig. 28, pl. 6, fig. 5; pl. 12, figs. 4, 5; stromatolite; Lower Cambrian or Vendian; Georgiana basin, Northern Territory, Australia.

GETAEIA Dragastan, (1972) 1973.

Getaeia pontica Dragastan, (1972) 1973 , p. 82-85, figs. 7-3; alga; Lower Cretaceous; northern basin of Babadag-Dobrogea, Rumania.

GINKGOITOCLADUS Krasilov, 1972.

Ginkgoitocladus burejense Krasilov, 1972 , p. $37-38$, fig. $5 \mathrm{a}-\mathrm{b} ; \mathrm{pl}$. 6 , figs. $1-4,8-10$; foliage, Ginkgoaceae; Lower Cretaceous; left bank of the Bureya river below the mouth of the Adnikan river, Kabarovsk Kray, U.S.S.R.

GLEDITSIOXYLON' Müller-Stoll and Mädel, 1967.

Gleditsioxylon columbian (Prakash and Barghoorn) Müller-Stoll and Mädel, 1967 , p. 148-149, wood, Leguminosae; Miocene; Columbia River near Vantage, Washington, U.S.A.

GLEICHENOIDES Kon'no, 1968.

Gleichenoides gagauensis Kon'no, 1968, p. 140-141, fig. LA-D; pl. 24, fig. 8; frond; Upper Mesozoic, Malaya.

GLOMEOPHYCUS Vologdin, 1962.

Glomeophycus filipendulus Vologdin, 1962 , pt. 2 , p. $499-500$, fig. 15 ; pl. 11, fig. 1; stromatolite, Vesiculariaceae; Lower Cambrian; basin of the upper course of the Lena, Manzurka river valley, U.S.S.R.

GLOSSOTHECA Surange and Maheshwari, 1970.

Glossotheca utakalensis Surange and Maheshwari, 1970, p. 180-181, figs. 1-4; pl. 40 , figs. $1-5$; pl. 41 , figs. 6-12; male fructification, Glossop- teridales, Upper Permian; Orissa, India.

GLOTTOLEPIS Bose and Srivastava, (1969) 1970.

Glottolepis migosa Bose and Srivastava (1969) 1970, p. 215-216, figs. la-c; pl. 1, figs. 1-9; scale leaves, incertae sedis; Lower Triassic; Nidpur, Sidhi district, Madhya Pradesh, India.

GOBICHARA Karczewska and Ziembinska-Tworzydlo, 1972.

Gobichara deserta Karczewska and Ziembinska-Tworzydlo, 1972, p. $72-75$, figs. 10,$11 ;$ pl. 15, figs. $1-5$; pl. 16, figs. $1-6$; pl. 19, fig. 1 ; fruit, Charophyta; lower Tertiary; Nemegt basin, Gobi Desert, Mongolia.

GORDONOPHYTON Korde, 1973.

Gordonophyton distinctum Korde, 1973 , p. 198-199, figs. 21, 47; pl. 40, fig. 4 ; pl. 41, fig. 1 ; algae, Epiphytaceae; Lower Cambrian; Bayankol river, Tuva and the southern Urals, U.S.S.R.

GORLOVELLA Vologdin, 1970.

Gorlovella obvoluta Vologdin, 1970, p. 1164, fig. 1a; unicellular microscopic algae; Vesiculariaceae; Precambrian; Shun'ga and Zaonezh'e villages, Karelo-Finnish SSR.

GOWERI Wolfe, 1968.

Goweri dilleri (Knowlton, 1900) Wolfe, 1968, p. 20-21, fig. 13, pl. 7, figs. 1,2 ; leaves, Menispermaceae; lower Tertiary; King County, Washington, U.S.A.

GRENULARIA Vologdin, 1970.

Grenularia borissovi Vologdin, 1970, p. 1165, fig. 1b; algae, Agamuseae; Precambrian; Shun'ga and Zaonezh'e villages, Karelo-Finnish SSR.

GRUNERIA Cloud and Semikhatov, 1969.

Gruneria biwabikia Cloud and Semikhatov, 1969, p. 140-142, pl. 3, figs. 1-3 (also Cloud, 1965, figs. 1, 2); stromatolite; Proterozoic; Minnesota, U.S.A.; Ontario, Canada; and western Australia.

GUPTIORACHIS Sharma, 1971.

Guptiorachis amarjolense Sharma, 1971 , p. 150-151, fig. 1 ; pl. $1 \mathrm{~A}-\mathrm{E}$; fern petioles; Middle Jurassic; Armajola, Rajmahal Hills, Bihar, India.

GUPTIOXYLON Sharma, 1969.

Guptioxylon amarjolense Sharma, 1969 , p. 145-153, table 1 ; figs. 1-5; pl. 26 , figs. $1-7$; pl. 27 , figs. 8-12; pl. 28, figs. 13-18; wood, having correlations with both Pentoxyleae and Medulloseae; Jurassic; Amarjola, Rajmahal Hills, India. 
GUSICHIA Chirkova-Zalesskaya, 1959. Gusichia furcata Chirkova-Zalesskaya, 1959, p. 76-77, fig. 56, sporangia, incertae sedis; Devonian; Ural-Volga areas, U.S.S.R.

GUTTOPORELLA Gnilovskaya, 1972.

Guttoporella densa Gnilovskaya, 1972, p. 132-134, fig. 55; pl. 15, fig. 3 ; alga, incertae sedis; Upper Ordovician; eastern Kazakhstan, U.S.S.R.

\section{$\mathrm{H}$}

HALLETHECA Taylor, 1971.

Halletheca reticulatus Taylor, 1971, p. 300-307, figs. 1-16; pteridosperm; Pennsylvanian; Berryville, Lawrence County, Illinois, U.S.A.

HARLANJOHNSONELLA Elliott, 1968.

Harlanjohnsonella annulata Elliott, 1968 , p. $494-495$, pl. 93 , figs. 1, 2; pl. 94, figs. 1, 2 ; algae, Dasycladaceae; Upper Cretaceous; Zlatibor area, southwestern Serbia, Yugoslavia.

HARRISOCARPON Chitaley and Namburdi, 1973.

Harrisocarpon sahnii Chitaley and Namburdi, 1973 , p. $36-41$, figs. $1-4$; pl. 1, figs. 1-6; dicotyledonous fruit, incertae sedis; ?uppermost Cretaceous; Mohgaon-kalan, Chhindwara district, Medhya Pradesh, India.

HARRISOSTROBUS Chitaley and Sheikh, 1973.

Harrisostrobus intertrappea Chitaley and Sheikh, 1973, p. 25-30, figs. $1-7$; pls. 10,11 ; gymnospermous cone; Deccan Intertrappean Series, uppermost? Cretaceous; Mohgaonkalan, Chhindwara district, Madhya Pradesh, India.

HELICONIAITES Trivedi and Verma, 1972.

Heliconiaites mahgoanensis Trivedi and Verma, 1972, p. 74-79, figs. $1-4$; pls. $46-48$, figs. $1-16$; monocotyledonous leaf; lower Eocene; Madhya Pradesh, India.

HEMIPHYLLUM Lemoine, 1969-70.

Hemiphyllum atacicum Lemoine, $1969-70$, p. $169-172$, pl. 3, figs. 1, 2 ; pl. 4, figs. 1,2 ; pl. 5, fig. 2; coralline algae; Cretaceous; southern France.

HERAKELLA Kochansky-Devidé, 1970.

Herakella paradoxa KochanskyDevidé, 1970, p. 21-25, pl. 5, fig. 1; pl. 12 , figs. $4-9$; pl. 13 , figs. $1-11$; pl. 14, figs. $1-13$; pl. 15 , figs. 1,2 ; algae; Middle Carboniferous; near
Ričice village, southern Croatia, Velebit mountains, Yugoslavia.

HETEROPORELLA Praturlon, 1966.

Heteroporella lepina Praturlon, 1966, p. 202-205, fig. 1 , pls. 51, 52; calcareous algae, Dasycladaceae; Upper Cretaceous; central Ápenninies, Italy.

HILLISTROBUS Chandler, 1966.

Hillistrobus axelrodi Chandler, 1966, p. 158-161, figs. 29-32; pls. 8, 9, figs. 80-87; cones, ?Taxodinae; Upper Jurassic; Utah, U.S.A.

HISSARELLA Sikstel', 1966.

Hissarella schamolensis Sikstel', 1966 , p. 25-27, pl. 10, figs. 1-11; pteridophyll, incertae sedis; Upper Permian and Lower Triassic; Khanaka river valley, Mt. Gissar, Tadzhik SSR.

HOLIGARNOXYLON Prakash and Awasthi, (1969) 1970.

Hologarnoxylon assamicum Prakash and Awasthi, (1969) 1970, p. 3536 , pls. 1-3, figs. 6-14; silicified wood, compared with modern wood, Anacardiaceae; Tertiary, near Jaipur, Assam, India.

HORNIELLA Shaykin, 1966.

Horniella robertsi (Peck, 1934) Shaykin, 1966, p. 158-160, fig. 2a, b; charophyte, Characeae; Columbia, Missouri, U.S.A.

\section{I}

IBYKA Shog and Banks, 1973.

Ibyka amphikoma Skog and Banks, 1973 , p. $366-378$, figs. $1-24$; protoarticulate; upper Middle Devonian; New York State, U.S.A.

IDANOTHEKION Millay and Eggert, 1970.

Idanothekion glandulosum Millay and Eggert, 1970, p. 51-58, figs. 1-24; ?pteridosperm male fructification; Middle Pennsylvanian; Williamson County, Illinois, U.S.A.

INGOXYLON Müller-Stoll and Mädel, 1967.

Ingoxylon sahnii (Ramanujam) Müller-Stoll and Mädel, 1967, p. 111-112; wood, Leguminosae; Miocene or Pliocene; southern India.

INOPINATELLA Elliott, 1971.

Inopinatella lawsoni Elliott, 1971, p. 637-641, figs. 1-6; pl. 120, figs. $1-3$; pl. 121, figs. $1-3$; algae, incertae sedis; Upper Silurian; near Aymestry Church, Shropshire, England.

INTERMURELLA Elliott, 1972.

Intermurella scotia Elliott, 1972, p. 361-362, pl. 3, fig. 3; pl. 4, figs. 
1-4; green algae, Dasycladaceae; Upper Ordovician; Girvan, Ayrshire, Scotland.

INTEXTULELLA Petryk, 1972.

Intextulella agglomerata Petryk, in Petryk and Mamet, 1972, p. 797, 798 , fig. 9 ; pl. 2, figs. 8 , 9; algae; Livingston Formation, Lower Carboniferous; southwestern Alberta, Canada.

INTUTICUTIS Schneider, 1969.

Intuticutis pulchra Schneider, 1969, p. 20,21 , pl. 5, figs. 1,2 ; cuticle of upper side of leaf, dicotyledon; middle Miocene; Upper Lusatia, east-central Germany.

ISOBERLINEOXYLON Lakhnapal and Prakash, 1970.

Isoberlineoxylon congoense Lakhnapal and Prakash, 1970 , p. 10-12, pl. 6, figs. 19-21; pl. 7, figs. 22, 24 ; wood, Leguminosae; Miocene; Lake Albert, Congo, east-central Africa.

ISODICTYOPTERIDIUM Rigby, 1972.

Isodictyopteridium walkomii Rigby, 1972, p. 9-10, figs. F, H, I ; ?fructification, incertae sedis; Permian; central Queensland, Australia.

ISOETODENDRON Bock, 1969.

Isoetodendron striata Bock, 1969, p. 51-52, figs. 97-101; treelike stem, Isoetales; Triassic; Winterpock, Virginia, U.S.A.

\section{$\mathrm{J}$}

JARENGA Vakhrameev, 1968.

Jarenga rosanovii Vakhrameev, 1968, p. $8-10$, figs. 1,2 ; pl. 1 , figs. $1-8$; fern; Middle Jurassic; Yarenga river, right tributary of the Vychergy River, northern part of the Russian platform, U.S.S.R.

$J E N S E N S I S P E R M U M$ Chandler, 1966.

Jensensispermum redmondi Chandler, 1966 , p. 151-156, figs. 14-28; pls. 5 , 6, figs. 47-74; seeds, ?Cycadophyte; Upper Jurassic; Utah, U.S.A.

\section{$\mathrm{K}$}

KADVOYA Korde, 1973.

Kadvoya mirabilis Korde, 1973, p. 141-142, fig. 25; pl. 17, figs. 3-5; pl. 18, fig. 1; algae, Kadvoyaceae; Lower Cambrian; Kadvoy river, eastern Tannu-Ola, Tuva, U.S.S.R.

KAMAENA Antropov, 1967.

Kamaena delicata Antropov, 1967, p. $123-124$, pl. 28, figs. 1-3; algae, Siphonales; Devonian and Lower
Carboniferous; central part of the east Russian platform, U.S.S.R.

KANDYRIA Sikstel', 1971.

Kandyria vasilkovskyi (Sikstel') Sikstel', in Sikstel' and others, 1971 , p. $67-69$, pl. 30, figs. 2-5; pl. 31 , figs. $1-6$; tree-fern; Carboniferous; left bank of the Angren river, Uzbekistan, U.S.S.R.

KANKAKEEA Pfefferkorn, 1973.

Kankakeea grundyi Pfefferkorn, 1973, p. 143-151, 2 figs., pls. 25, 26 ; buds for vegetative reproduction, ferns; Carboniferous; Illinois and Indiana, U.S.A.

KARINOPTERIS Boersma, 1972.

Karinopteris daviesii (Kidston) Boersma, 1972 , p. 73-74, 82, 129 134, figs. 14a, b; pls. 28-31; for Mariopteris daviesii Kidston, 1925, p. 641, pl. 146, figs. $1,1 \mathrm{a}$; fernlike foliage; incertae sedis; Carboniferous; South Wales.

KATASIOPTERIS Radchenko, 1967.

Katasiopteris lebedevii Radchenko, in Vladimirovich and others, 1967, p. 26-27, fig. 5; fern; Lower Triassic; Kuznets and Tunguska basins, central Siberia, U.S.S.R.

KATERNIA Cloud and Semikhatov, 1969.

Katernia africana Cloud and Semikhatov, 1969, p. 1046-1047, pl. 3, figs. 4, 5, (also illus. in Young, 1932 , 1934 and Young and Mendelsson, 1948) ; stromatolite; Proterozoic; South Africa and Medicine Bow Mts., Wyoming, U.S.A.

KAULANGIOPHYTON Gensel and Kasper, 1969.

Kaulangiophyton akantha Gensel and Kasper, in Gensel, Kasper and Andrews, 1969 , p. 265-275, figs. 1-8; branches, shoots with fertile sporangia, Zosterophyllophytina of Banks; Lower Devonian; northern Maine, U.S.A.

KCHONOMAKIDIUM Shevedov, 1962.

Kchonomakidium srebrodolskae Shvedov, 1962, p. 59-61, fig. 1; pl. 1, figs. 1-6; pteriodophyll, incertae sedis; Lower Triassic; Khantayskoye lake, Noril'sk region, Kransnoyarskiy Kray, U.S.S.R.

KEEGA Wray, 1967.

Keega australe Wray, 1967, p. 16-19, fig. 6 ; pl. 3 , figs. $1-6$; algae, incertae sedis; Upper Devonian; Canning basin, Western Australia.

KENELLA Korde, 1973.

Kenella ornata Korde, 1973, p. 216, fig. 9 ; pl. 65 , fig. 4 ; pl. 66 , fig. 1 ; algae, Kenellaceae; Abakan river basin, Srednyaya Kenya river, western Sayan, U.S.S.R. 
KENELLA Samylina, 1968.

Kenella harrisiana Samylina, 1968, p. 211-212, fig. 2G: pl. 1, figs. 11-13; fruit, incertae sedis; Lower Cretaceous; Omuskchan, Kolyma basin, eastern Siberia, U.S.S.R.

KETOVIA Vladimirovich, 1972. Ketovia furcata Vladimirovich, 1972 , p. 96 , pl. 2 , figs. 1,4 ; fernlike foliage; Middle Triassic; Orenburg, Ural region, U.S.S.R.

KOILOSPHE'NUS Bohlin, 1971.

Koilosphenus cuneifolius Bohlin, 1971 , p. 47-48, pl. 7, fig. 4; drawing pl. 81-90, figs. $90 \mathrm{~A}-\mathrm{D}$; shoot with apex and foliage, Noeggerathiales; upper Paleozoic; Yüerhhung, Kansu, China.

KOLYME'LLA Samy'lina and Filippova, 1970.

Kolymella raevskii Sam'ylina and Filippova, 1970 , p. 92-94, figs. 1, 2 ; pl. 12 , figs. 6,7 ; fern, ?Pteridaceae; Cretaceous; northeastern U.S.S.R.

KOSTINOPHYCUS Vologdin, 1962.

Kostinophycus irregularis Vologdin, 1962 , pt. 2, p. 493-494, fig. 10 ; pl. 10, fig. 2; algae, Sarmaellaceae; Lower Cambrian; right bank of the Yenisey, above Kostino village, Krasnoyarskiy Kray, Turukhansk region, U.S.S.R.

KOSVOPHYTON Korde, 1973.

Kosvophyton uralicum Korde, 1973, p. 206-207, pl. 64, fig. 1; algae, Epiphytaceae; Upper Silurian and Lower Devonian; Kos'va river, northern Urals; right bank of the Kolonga river, $0.5 \mathrm{~km}$ beyond the mouth, southern Urals, U.S.S.R.

KOVALIA Teslenko, 1970.

Kovalia grandifolia Teslenko, 1970, p. $183-185$, pl. 48 , figs. 1 , 2 ; pl. 52 , fig. 3 ; leaves, incertae sedis; Jurassic; right bank of the Tom river, Kuznets basin, U.S.S.R.

KRAUSELCLADUS Yoshida, 1970.

Kräuselcladus canoinhensis Yoshida, 1970, p. 7-10, pl. 1, figs. 1-3; pl. 2, figs. 1-4; conifer shoots; Permian; northern Santa Catarina State, Brazil.

KRAUSELICUTIS Schneider, 1969.

Krauselicutis punctata Schneider, 1969 , p. 23-24, pl. 7, figs. 4, 5; cuticle, ?Magnoliales; middle Miocene; Lower Lusatia, east-central Germany.

KREJCIELLA Obrhel, 1968.

Krejciella putzkeri Obrhel, 1968b, p. 463-464, pl. 1, figs. 1-5; query, lycopod; Ordovician; Praha-Dolní Sárka-Tal, riördl. vom Friedhof, central Bohemia, Czechoslovakia.
KRITHODEOPHYTON Edwards, 1968.

Krithodeophyton croftii Edwards, 1968 , p. 684 , pls. $130-132$, branching axes, fructification, Enigmophyton; Lower Devonian; South Wales, Great Britain.

KUNDATIA Korde, 1973.

Kundatia composita Korde, 1973, p. 120-121, fig. 2 ; pl. 7, figs. $1-5$; pl. 8, fig. 1; algae, Kundatiaceae; Lower Cambrian; Kiya river, Kuznetskiy Alatau, U.S.S.R.

KUPRIANOVAITES Namburdiri, 1969.

Kuprianovaites deccanii Namburdiri, 1969, sporocarp, incertae sedis; Paleocene; Mohgaon Kalan, Chhindeara district, Madhya Pradesh, India.

KYMALITHON Lemoine and Emberger, 1967.

Kymalithon belgicum (Fosile, 1909) Lemoine and Emberger, 1967, p. 3-14, pls. 1-4; algae; Lower Cretaceous; southwestern France.

KYZASSIA Korde, 1973.

Kyzassia formosa Korde, 1973, p. 123-124, pl. 9, figs. 1, 2; algae, Cornutulaceae; Lower Cambrian; western Sayan, U.S.S.R.

\section{L}

LACUNIDERMA Krasilov, 1973.

Lacuniderma meyenii Krasilov, 1973a, p. 109, pl. 22, figs. 41-48; cuticle, incertae sedis; Upper Cretaceous; town of Mgachi, western coast of northern Sakhalin.

LAMELLOPHYCUS Vologdin, 1962.

Lamellophycus aculeatus Vologdin, 1962 , pt. 1 , p. $180-185$, figs. $15-17$; pl. 12, figs. 1-4; stromatolite, Lermontovaephycaceae; middle $\mathrm{Si}$ nian; right bank of the Nizhney Tunguska, second Kamen' promontory, U.S.S.R.

LAMELLOSTROMA Vologdin, 1962.

Lamellostroma vesculara Vologdin, 1962 , pt. 1 , p. 303 , 305, figs. 82 , 83a; pl. 74. fig. 1; stromatolite, Lamellostromataceae; Lower Cambrian; Borlog river, southwestern Cisbaikal, Burul'deyka river basin, U.S.S.R.

LANGCAMIA Srebrodolskaya, 1969.

Langcamia zeilleri Srebrodolskaya, 1969 , p. $91-94$, fig. 4 ; pl. 2 , figs. 3, 4; foliage, Filicinae, incertae sedis; Upper Triassic; northern Viet Nam.

LANGOXYLON Stockmans, 1968.

Langoxylon asterochlaenoideum Stockmans, 1968, p. 25-29, fig. 6; 
pl. 10, figs. 1-1d; pl. 11, figs. 2, 3; pl. 12, figs. 1-8; wood, coenopteris; Middle Devonian; Brabant Massif region, central Belgium.

$L A N N E O X Y L O N$ Prakash and Tripathi, 1967.

Lanneoxylon grandiosum Prakash and Tripathi, 1967, p. 462-463, figs. 1-3; wood, Anacardiaceae; Tertiary; Mikir hills, Assam, India.

LAPPACARPUS Douglas, 1969.

Lappacarpus aristata Douglas, 1969, p. 225-228; figs. IV: 5-6; pl. 42 , figs. 5,8 ; pl. 43 , figs. $1-5$; pl. 44 , fig. 2 ; reproductive organ, angiosperm; Lower Cretaceous; Yangery Bore, Victoria, Australia.

LASIOSTROBUS Taylor, 1970.

Lasiostrobus polysacci Taylor, 1970, p. 670-688, figs. 1-48; gymnosperm male cone; Upper Pennsylvanian; Lawrence County, southeastern Illinois, U.S.A.

$L A T E R I C U T I S$ Roselt and Schneider, 1969.

Latericutis fragmentata Roselt and Schneider, 1969, p. 62, pl. 9, fig. 5 ; cuticle, monocotyledon, Palmae of Glumiflorae; Miocene; Lower Lusatia, East Germany.

LATICAULINA Krasilov, 1970.

Laticaulina papillosa Krasilov, 1970 , p. 141-142, pl. 11, figs. 1-3; leafy liverwort, Jungermanniales; Upper Jurassic; right bank of the Bureya river, Khabarovsk Territory, eastern Siberia, U.S.S.R.

$L A U R A C E O P H Y L L O D E R M A$ Giessen, 1971.

Lauraceophylloderma ebenoides (Engelhardt) Giessen, 1971, p. 49-50, fold-in pl. 9 ; figs. 1a-c; pl. 13, figs. $6-8$; pl. 14, figs. $1-3$; leaf; Lauraceae; Eocene; near Darmstadt, West Germany.

LAUSATICUTIS Roselt and Schneider, 1969.

Lausaticutis rugosa Roselt and Schneider, 1969 , p. 66-67, fig. 17; pl. 11 , figs. $3-5$; cuticle of an undetermined angiosperm; middle Miocene; Klettwitz, Lower Lusatia, East Germany.

LECLERCQIA Banks, Bonamo and Grierson, 1972.

Leclercqia complexa Banks, Bonamo and Grierson, 1972, p. 35-36, pl. 1 figs. 1-48; slender herbaceous lycopod; Protolepidodendraceae; Panther Mountain Formation; Schoharie County, New York, U.S.A.

LEGRANDIA Stockmans, 1968.

Legrandia sporangifera Stockmans,
1968, p. 36-37, pl. 6, figs. 7, 7a; compared with Pseudosporochnus, Psilophytales; Middle Devonian; Brabant Massif region, central Belgium.

$L E G U M I N A N T H U S$ Kräusel and Schaarschmidt, 1966.

Leguminanthus siliquosus Kräusel Schaarschmidt, 1966, p. 41-42, pl. 11 , figs. $1-5 ;$ pl. 12 , figs. $1-9$; pl. 13, figs. 1-11; pl. 14, figs. 1-20; pl. 15, figs. 1-19; bennettitalean pollen organ; Middle Triassic; Switzerland.

LELSTOTHECA Maheshwari 1972; an anagram for Stellotheca Surange and Prakash, 1962, established as a new combination with Phyllotheca robusta Feistmantel, 1880.

Lelstotheca robusta (Feistmantel, 1880) Maheshwari, 1972, p. 106; equisetaceous foliage; Lower Permian; Rajmahal Hills, Bihar, India.

LEPTOCYCAS Delevoryas and Hope, 1971.

Leptocycas gracilis Delevoryas and Hope, 1971, p. 3-7, figs. 1-12; stems, Cycadaceae; Upper Triassic; Chatham County, North Carolina, U.S.A.

LEPTORICHOMARIA Vologdin, 1962.

Leptotrichomaria intermissa Vologdin, 1962, pt. 1, p. 255-258, figs. 54, 55; pl. 50, fig. 1 ; pl. 51, figs. 1-3; algae, Trichostromataceae; Lower Cambrian and Sinian; Turukhansk region, right bank of the Yenisey near Miroedikh village, and near Kostino village, below the mouth of the Sukhaya Tunguska, central Siberia, U.S.S.R.

LERICHEA Stockmans, 1968.

Lerichea krystofovitchii Stockmans, 1968 , p. 12-13, pl. 9, figs. 2, 2a; branches, Psilophytales; Middle Devonian; Brabant Massif region, central Belgium.

LERMONTOVAEPHYCUS Vologdin, 1962.

Lermontovaephycus lamellosus Vologdin, 1962, pt. 1, p. 166-167, 172176, figs. 10 , 11; pl. 7, fig. 1 ; pl. 8 , fig. 1 ; pl. 9 , figs. 1,2 ; stromatolite, Lermontovaephycaceae; $\mathrm{Si}$ nian right bank of the Angara river, U.S.S.R.

LEUTHARDTIA Kräusel and Schaarschmidt, 1966.

Leuthardtia ovalis Kräusel and Schaarschmidt, 1966, p. 26-27, figs. 6 , 7; pl. 8, figs. $1-5$; pl. 9, figs. 1-5; bennettitalean pollen organ; Middle Triassic; Switzerland. 
LEVICUTIS Roselt and Schneider, 1969.

Levicutis complitata Roselt and Schneider, 1969 , p. 59, pl. 7, fig. 2; cuticle of leaf sheath, [Graminales] : Miocene; Upper and Lower Lusatia, East Germany.

LIDASIMOPHYTON Senkevich, 1961.

Lidasimophyton akkermensis Senkevich, 1961, p. 155-169, fig. 6; pl. 24 , figs. $2-5$; pl. 26 , figs. $1-5$; lycopod, Drepanophycaceae; Middle Devonian, western Pribalkhash, southern Kazakhstan, U.S.S.R.

LILPOPIA (Lilpop, 1937) Conert and Schaarschmidt, 1970.

Lilpopia raciborskii (Lilpop, 1937) Conert and Schaarschmidt, 1970. A new name for Tristachya Lilpop; p. 793-794; Lilpopiaceae; Permian and Carboniferous; 35 $\mathrm{km}$ from Cracow, Poland.

LIMNOBIOPHYLLUM Krasilov, 1973.

Limnobiophyllum scutatum (Dawson, 1875) Krasilov, 1973a, p. 110111 , pl, 23, figs. 49-61, cuticle, incertae sedis; Upper Cretaceous; near the mouth of Bureya river, Tsagajan, Amur province, U.S.S.R.

LIRIODENDROXYLON Prakash, Březinová and Bůžek, 1971.

Liriodendroxylon tulipferum Prakash, Březinová and Bůžek, 1971, p. 108-110, pl. 35, figs. 26-29, pl. 36, figs. 30-34; wood Magnoliaceae; Oligocene; Doupovské hory mountains, northern Bohemia, Czechoslovakia.

LOBIFOLIA Lebedev and Rasskazova, 1968.

Lobifolia povorokovskii (Prynada in Vachrameev and Doludenko, 1961) Lebedev and Rasskazova, 1968, p. 61-63, figs. 2,3 ; pl. 1, figs. 1-3; fern; Lower Cretaceous; Bureya river basin, U.S.S.R.

LOPATINELLA Vologdin, 1962.

Lopatinella bipartita Vologdin, 1962, pt. 1, p. 206-209, fig. 27; pl. 35, figs. 1,2 ; pl. 26 , figs. 1,2 ; stromatolite; Lopatinellaceae; middle Sinian; right bank of the Nizhney Tunguska, about $34 \mathrm{~km}$ from the mouth, U.S.S.R.

LOVOAXYLON Louvet, 1967.

Lovoaxylon princeps Louvet, 1967, p. 186-197, 2 figs., 1 pl., 1 table; wood; Algeria, northern Africa.

LUCERNELLA Grambast and Lorch, 1968.

Lucernella ampullacea Grambast and Lorch, 1968, p. 48-50, pl. 1, figs. 1a-d, 2a-d, 3a-d; pl. 2, figs. 1a-9; charophyte, Clavatoraceae; Lower Cretaceous; southern Lebanon.
LUDLOVIA Korde, 1973.

Ludlovia multispora Korde, 1973, p. 207-209, fig. 62 ; pl. 65, fig. 1 ; pl. 66, fig. 1; algae, Epiphytaceae; Upper Silurian; left bank of Sos'va river, eastern slope of the northern Urals, U.S.S.R.

LUTUGINIA Prynada and Radchenko, 1967.

Lutuginia furcata Prynada and Radchenko, in Vladimirovich and others, 1967, p. 28-29, fig. 11, coniferous branch with shoots; Lower Triassic; Kuznets basin, central Siberia, U.S.S.R.

LYCHNOTHAMNITES Maslov, 1966.

Lychnothamnites naryensis Maslov, 1966 , p. $77-79$, fig. 26 ; pl. 9 , figs. 9-11; charophyte; middle Pliocene; Naryn depression, Kirghiz SSR.

LYSVAELLA Tchuvashov, 1971.

Lysvaella partita Tchuvashov, 1971, p. 86-89, fig. 1 ; pl. 10 , figs. $1-4$; algae, Corallinaceae; Lower Permian; Chusovoy river basin, western slope of the Urals, U.S.S.R.

\section{M}

\section{MADIGANITES Walter, 1972.}

Madiganites mawsoni Walter, 1972 , p. 158-161, figs. 8,47 , pl. 1 , figs. 1,2 ; pl. 28, figs. 1-3; stromatolite; Middle and Upper Cambrian; Waterhouse and MacDonald Ranges, Australia.

\section{MAGARIELLA Page, 1973.}

Margariella cretacea Page, 1973, p. 572-575, figs. 1-15; coniferal stem with leaves; ?Taxodiaceae; Upper Cretaceous; central California, U.S.A.

MAGNICUTIS Schneider, 1969.

Magnicutis glandulosa Schneider, 1969 , p. 17-18, fig. 2; pl. 3, figs. 6, 7; cuticle, Loranthaceae; Miocene; Upper Lausatia, east-central Germany.

$M A G N I F E R O X Y L O N$ Awasthi, (1965) 1966.

Magniferoxylon scleroticum Awasthi, (1965) 1966, p. 131-135, figs. 1-5; pl. 1, figs. $1-4$; pl. 2 , figs. 5-11; wood, Anacardiaceae; Tertiary; South Arcot district, Madras, India.

MAJSASSIA Sukhov, 1969.

Majsassia elliptica Sukhov, 1969, p. 176 , pl. 31, figs. 1-3; gymnosperm seed; Lower Carboniferous; Kuznets basin, central Siberia U.S.S.R.

MALOIDOVYLON Grambast-Fessard 1966.

Maloidoxylon castellanense Grambast-Fessard, 1966, p. 138-145, 
figs. $3,4,6$; pl. 13 (17), figs. 1-3; pl. 14 (18), figs. 1-5; wood, Rosaceae; Miocene and Pliocene; Castellane, Basses-Alpes, southeastern France.

MANSURKELLA Vologdin, 1962.

Mansurkella densa Vologdin, 1962, pt. 2, p. $490-491$, fig. 8, pl. 9, figs. 2,3 ; stromatolite, Sarmaellaceae; Lower Cambrian; Manzurka river basin, U.S.S.R.

MOAKHEOPTERIS Srebrodolskaya, 1969.

Maokheopteris vietnamica Srebrodolskaya, 1969. p. 89-91, pl. 2, fig. 2 ; pl. 3 , figs. 1, 2; foliage, Filicinae, incertae sedis; Upper Triassic; northern Viet Nam.

MARCOUIA Ash, 1972.

Marcouia neuropterides (Daugherty) Ash, 1972, p. 423-428, fig. $1 \mathrm{~A}-\mathrm{E}$; pl. 80, figs. 1-9; fernlike foliage; incertae sedis; Upper Triassic; New Mexico and Arizona, U.S.A.

MARENITA Korde, 1973.

Marenita kundatica Korde, 1973, p. 109-110, pl. 1, fig. 3; algae, Marenitaceae; Lower Cambrian; Kiya river, $1 \mathrm{~km}$ below the mouth of the Kundat river, Kuznetskiy Alatau, Tuva, U.S.S.R.

MARGARIELLA Page, 1973.

Margariella cretacea Page, 1973, p. 572-575, figs. 1-15; coniferal stem with leaves; ?Taxodiaceae; Upper Cretaceous; central California, U.S.A.

MASLOVINA Obrhel, 1968.

Maslovina meyenii Obrhel, 1968a, p. $367-370$, fig. $1 \mathrm{~A}-\mathrm{D}$; pl. 1 , figs. $1-$ 6 ; pl. 2, figs. 1-5; algae; Codiaceae; Silurian; Bohemia, Czechoslovakia.

MATIA Townrow, 1967.

Matia podocarpoides (Ettingshausen, 1891) Townrow, 1967 , p. 125-129, 131,133 , pls. Ij, IIg; foliage and seed cone, Podocarpaceae; Middle Jurassic; New Zealand, and Queensland, Australia.

$M E L I A C E A E P H Y L L U M$ Varma, 1968.

Meliaceaephyllum mahagonites Varma, 1968, p. 83-85, pl. 1, fig. 1; leaf impression, Meliaceae; upper Miocene (middle Shivaliks) ; Hardwar, Uttar Pradesh, India.

MELLPORELLA Rácz, (1964) 1966.

Mellporella anthracoporellaformis Rácz, (1964) 1966, p. 99-100, pl. 4, figs. 4-6; calcareous green algae, ?Dasycladaceae: the San Emiliano and Lois-Ciguera Formations, Carboniferous; Leon province, northwestern Spain.
MENAISPERMAE Pettitt and Lacey, 1972.

Menaispermae greenlyii Pettitt and Lacey, 1972, p. 154-169, figs. 1, 2; pls. 1-4; gymnospermous seed compression; Late Visean, Lower Carboniferous; Menai Straits, Caernarvonshire, North Wales.

MENSELINA Antropov, 1967.

Menselina clathrata Antropov, 1967, p. 124, pl. 28, figs. 4-6; ?algae; ?Rhodophyta; Lower Carboniferous; central part of the east Russian platform, U.S.S.R.

MENUCOA Petriella, 1969.

Menucoa cazaui Petriella, 1969, p. 293-320, figs. 1-3; pl. 1, figs. 1-4; cycad stems, Cycadales; lower Tertiary; Los Menucos, Rio Negro province, Argentina.

MESOLARIX Jähnichen and Kahlert, 1972.

Mesolarix mongolica Jähnichen and Kahlert, 1972, p. 973-4, 979, pl. 4, fig. 3 ; pl. 5 , fig. 3 ; pl. 6 , fig. 6 ; sprout fragments with dwarf shoots; Upper Jurassic and Lower Cretaceous; environs of Bajanchonger, Mongolian People's Republic.

METAKAMAENA Endô, 1969.

Metakamaena gracilis Endô, 1969, p. $81-82$, fig. 4 ; pl. 5, figs. 1 , 2 ; algae, compared with Calcisphaera; Permian; Thailand.

MILLARIA Pflug, 1966.

Millaria implexa Pflug, 1966, p. 6667 , pl. 28, figs. 6-18, 20-44, 48-63, pl. 29, figs. 1-28; ?algae, ?Cyanophyta; Idaho-Montana, U.S.A.

MIMOSACEOXYLON Lakhnapal and Prakash, 1970.

Mimosaceoxylon lebacqii Lakhnapal and Prakash, 1970, p. 8-10, pl. 4, figs. 14, 15; pl. 5, figs. 16-18; wood, Leguminosae; Miocene; Lake Albert, Congo, east-central Africa.

MIMOSOXYLON Müller-Stoll and Mädel, 1967.

Mimosoxylon tenax (Felix) MüllerStoll und Mädel, 1967, p. 134-136, fig. 9 ; pl. 35, figs. 68-71; wood, Leguminosae; Upper Cretaceous; near Oaxaca, Mexico.

MIRELLA Samylina, 1967.

Mirella borealis Samylina, 1967, p. 157-158, pl. 14, figs. 1a, 2-6; mega and microsporangia, incertae sedis; Lower Cretaceous; right bank of the Zyrianska river, Zyrianska coal basin, Yakut SSR.

MITRAGYNAXYLON Koeniguer and Lemoigne, 1973.

Mitragynaxylon gevini Koeniguer and Lemoigne, in Gevin, Koeniguer 
and Lemoine, 1973, p. 386-388, figs. 1, 2; pl. 23, figs. 1-8; wood, Rubiaceae; Oligocene and Miocene; Tindouf region, Algeria.

MONILIPORELLA Gnilovskaya, 1972.

Moniliporella camerata Gnilovskaya, 1972 , p. $102-106$, fig. 45 ; pl. 10 , figs. 1-3; pl. 11, figs. 1, 2; alga, Moniliporellaceae; Upper Órdovician; eastern Kazakhstan, U.S.S.R.

MONOSTYCHIA Vologdin, 1962.

Monostychia lapidosa Vologdin, 1962 , pt. 2 , p. 500-502, fig. 16 ; pl. 13 , figs. 1, 2; stromatolite, Vesculariaceae; Lower Cambrian; basin of the upper course of the Lena, Manzurka river near Polosco village, U.S.S.R.

MOROPHYLLUM Kirichkova and Budantsev, 1967.

Morophyllum denticulatum Kirichkova and Budantsev, 1967, p. 941942, pl. 2, figs. 7-14; angiosperm leaves; Lower Cretaceous; Sitte river, western tributary of the Lena, Yakutskaya, U.S.S.R.

MOYLIOSTROBUS Miller and Brown, 1973.

Moyliostrobus taxanum Miller and Brown, 1973, p. 564-569, figs. 113; voltzialean cone; Permian; Brewster County, Texas, U.S.A.

MUCILINA Korde, 1973.

Mucilina fossilis Korde, 1973, p. 118119 , fig. 3 ; pl. 6 , figs. $1-4$; algae, Mucilinaceae; Lower Cambrian; Kuznetskiy Alatau and Batenev ridge, U.S.S.R.

MULLEROXYLON Page, 1970.

Mulleroxylon eupomatioides Page, 1970 , p. 1143 , figs. 12-14; angiospermous wood; Upper Cretaceous ; Stanislaus County, central California, U.S.A.

MYRTOIDOXYLON Gottwald, 1966.

Myrtoidoxylon noldeae Gottwald, 1966 , p. $85-87$, pl. 23 , fig. 19 ; pl. 24, figs. 20-22; wood, Myrtaceae; middle Eocene; Tagebau near Helmstedt, Westdeutschland.

\section{$\mathrm{N}$}

NANSENIA Sveshnikova and Budantsev, 1967.

Nansenia arctica Sveshnikova and Budantsev, 1967, p. 124-125, pl. 12 , figs. 1-6; stem with leaves, Pinaceae; Lower Cretaceous; Franz Josef Land.

NEMEGTICHARA Karczewska and Ziembinslia-Tworsydło, 1972.

Nemegtichara prima Karczewska and Ziembinska-Tworzydło, 1972, p. 54-57, figs. 2, 3; pl. 7, figs. 1, 3, 4; pl. 8 , figs. $1,2,5,6$; pl. 9, fig. 2; pl. 24 , figs. $1,3,4$; fruit, Charophyta; lower Tertiary; Nemegt basin, Gobi desert, Mongolia.

NEMEJCOPTERIS Barthel, 1968.

Nemejcopteris feminaeformis (Schloth.) Barthel, 1968, p. 727733 , pl. 1, figs. $1-15$; pl. 2, figs. 111 ; pl. 3 , figs. $1-13$; pl. 4 , figs. $1-$ 9 ; fern, Zygopteridaceae; Lower Permian; Döhlen basin, east-central Germany.

NEODASYPORELLA Endô, 1969.

Neodasyporella innerannulata Endô, 1969 , p. $40-41$, fig. 2 ; pl. 36, figs. 1,2 ; pl. 42, fig. 5 ; algae, Corallinaceae; Permian; Thailand.

NERUSIANDELLA Vologdin, 1962.

Nerusiandella faveoleta Vologdin, 1962 , pt. 1 , p. $284-285$, fig. 69 , pl., figs. 1, a, 2, a; stromatolite, Plexostromataceae; middle Sinian; right bank of the Nizhney Tunguska about $34 \mathrm{~km}$ from its mouth; Turukhansk region, U.S.S.R.

NEUBURGIA M. I. Radchenko, 1969.

Neuburgia karutauensis M. I. Radchenko, 1969, p. 106-108, pl. 1 figs. 1-4; pl. 2 figs. 1-6; fragments of leaf impressions, compared with Anisopteris and Rhacopteris; Lower Carboniferous; southern Kazakhstan, U.S.S.R.

NIAYSSIOIDEA Stockmans, 1968.

Niayssioidea belgica Stockmans, 1968, p. 14-16, fig. 2; pl. 2, figs. $8,8 \mathrm{a}$; stem, ?Psilophytale; Middle Devonian; Brabant Massif region, central Belgium.

NICHOLSONIA Korde, 1973.

Nicholsonia glomerata Korde, 1973, p. $212-215$, pl. 64 , fig. 1 ; pl. 65 , fig. 1; algae, Kenellaceae; Lower Cambrian; Shangan river, Kuznetskiy Alatau; Batenev ridge, Tuva; area of Sukhaya Erba village, northeast of Dolgiy Mys mountain, U.S.S.R.

NIDIA Bose and Srivastava, 1973.

Nidia ovalis Bose and Srivastava, 1973 , p. $75-78$, figs. $4 A$, 6-8; pl. 2 , figs. $9-13$; pl. 3 , figs. 16,17 ; female cone, incertae sedis; Lower Triassic; Gopad river valley, Nidpur, Sidhi district, Madhya Pradesh, India.

NIDISTROBUS Bose and Srivastava, (1972) 1973.

Nidistrobus harrisiana Bose and Srivastava, (1972) 1973, p. 211212 , pl. 1 , figs. $1-5$; male fructification; incertae sedis; Lower Triassic; Gopad river valley, Nidpur, Sidhi district, Madhya Pradesh, India. 
NOTHODACRIUM Townrow, 1967.

Nothodacrium warrenii Townrow, 1967, p. 137-141, figs. 1A-D, 2A$\mathrm{E}, 3 \mathrm{~B}-\mathrm{D}$; pl. 1 , figs. A-B, D; coniferous shoot with base of seed cone, Podocarpaceae; Jurassic; east Antarctica.

NOTOCALAMITES Rigby (1970) 1972.

Notocalamites askosus Rigby (1970) 1972, p. 161-163, fig. 1 ; pl. 1 , fig. 1 ; fructification on stems, Notocamamitaceae; upper Paleozoic; Santa Catarina, Brazil.

NOVANTIELLA Elliott, 1972.

Novantiella ordoviciana Elliott, 1972, p. 362-363, pl. 6, figs. 1, 2; green algae, Dasycladaceae; Upper Ordovician; Girvan, Ayrshire, Scotland.

NYMPHAEOCAULON Trivedi and Ambwani, 1971.

Nymphaeocaulon intertrappeum Trivedi and Ambwani, 1971, p. 129136 , figs. $1-3$; pls. 44-45, figs. 113; fossil axis, Nymphaeaceae; lower Tertiary; Mohgaonkalan, Madhya Pradesh, India.

\section{0}

OMPHALEAEPHYLLUM Rásky, (1965) 1966.

Omphaleaephyllum weylandi Rásky, (1965) 1966, p. 266-267, pl. 2, fig. 6; leaf impression, incertae sedis; Tertiary; Ipolytarnoc, Hungary.

OPILIOXYLON Koeniguer, 1970.

Opilioxylon nigerinum Koeniguer, 1970 , p. $148-151$, fig. $3: 1-3$; paleoxylotomous wood; Upper Cretaceous; Mount Kanak, Republic of Niger, western Africa.

ORCHIDACITES Straus, 1969.

Orchidacites orchidioides (Straus,
1954) Straus, 1969 , p. $167 ;$ pl. 28 ; fig. 2 ; capsule, Orchidaceae; Pliocene; Willershausen, Germany.

ORDINICUTIS Roselt and Schneider, 1969.

Ordinicutis orbirima Roselt and Schneider, 1969, p. 78-79, fig. 28, pl. 20, figs. 3,4 ; cuticle of undetermined dicotyledon; Miocene; Frielendorf, Hessen, East Germany.

ORTHRIOSIPHONOIDES Petryk, 1972.

Orthriosiphonoides salterensis Petryk, in Petryk and Mamet, 1972, p. 776, fig. 3 ; pl. 2, figs. 10-14; pl. 3 , figs. 4, 5; algae; Visean, Lower Carboniferous; southwestern Alberta, Canada.

OVIDOPHYCUS Vologdin, 1962.

Ovidophycus titorenko Vologdin,
1962 , pt. 2 , p. $496-498$, fig. 13 ; pl. 11, fig. 5 ; algae, Tumidophyceae; Lower Cambrian; Manzurka river valley, upper course of the Lena, Kachugsk area, Ikurtsk Oblast, U.S.S.R.

$\mathbf{P}$

\section{PALAEOCALLITROXYLON Gregus, 1970.}

Pallaeocallitroxylon limburgense Greguss, 1970, p. 270-271, pl. 1, figs. 2, 4-5, 8-9; pl. 2, figs. 13, 1516; wood, Cupressaceae; Tertiary; Limburg, southeastern Netherlands.

PALEOERICOMA Elias, 1942.

Paleoericoma hitchcocki Elias, 1942, p. 100-101, pl. 15, figs. 7, 8; hulls, compared with the living section Ericoma of Oyzopsis; Pliocene; Yuma County, Colorado, U.S.A.

PALAEOPHRAGMITES LeMone and Johnson, 1969.

Palaeophragmites gilei LeMone and Johnson, 1969, p. 79, pl. 1, fig. 1; pl. 3, fig. 1 ; culms, Gramineae; Neogene; Rincon hills, Dona Ana County, New Mexico, U.S.A.

PALEOPHYLLUM Endô, 1968.

Paleophyllum hashimoto Endô, 1968, p. 213, pl. 35, fig. 4; algae, Corallinaceae; Mansalay Formation, Jurassic; Mindoro Island, Philippines.

PALAEOSMUNDA Gould, 1970.

Palaeosmunda williamsi Gould, 1970, p. 13-21, figs. 2, 3; pl. 1, figs. 1, 2 ; pl. 2, figs. 1-7; pl. 3 , figs. 1-8; pl. 4 , figs. 1-10; trunks and rhizomes, Osmundaceae; Upper Permian; Bowen basin, Queensland, Australia.

PALMATOPORELLA Gnilovskaya, 1972.

Palmatoporella lata Gnilovskaya, 1972 , p. 136-137, fig. 57; pl. 15, fig. 5 ; alga, incertae sedis; Upper Ordovician, eastern Kazakhstan, U.S.S.R.

PANOMNIELLA Kolosov, 1966.

Panomniella ornata Kolosov, 1966, p. 978-979, fig. 1A; algae, Rivulariaceae; Precambrian; Olekma river basin, Yakut SSR.

PAPULOPHYCUS Vologdin, 1962.

Papulophycus pennatus Vologdin, 1962 , pt. 1 , p. 280-283, fig. 67 ; pl. 64, figs. 1, 2; stromatolite, plexostromataceae; upper Sinian; Kachergat river on the southwestern shore of Lake Baikal, U.S.S.R.

PARACACIOXYLON Müller-Stoll and Mädel, 1967. 
Paracacioxylon odonellii (Menéndez) Müller-Stoll und Mädel, 1967, p. 137-138; wood, Leguminosae; Tiopunco, Tucuman province, Argentina.

PARACHABKOVIA Korde, 1973.

Parachabkovia dura Korde, 1973, p. 157-158, pl. 29, fig. 3; algae, Parachabkoviaceae; Lower Cambrian; Sanashtykgol river, western Sayan, U.S.S.R.

PARAEPIPHYTON Wray, 1967.

Paraepiphyton caritus Wray, 1967, p. $41-43$, fig. 15 ; pl. 8, figs. $1-15$; algae, incertae sedis; Upper Devonian; Canning basin, Western Australia.

PARAPHYLLUM Lemoine, 1969-70.

Paraphyllum amphiroaeforme (Rothpletz) Lemoine, 1969-70, p. 177181, pl. 12, fig. 1; pl. 13, fig. 2; coralline algae; Cretaceous; southern France.

PARAPINUXYLON Huard, 1966.

Parapinuxylon hostensianum Huard, 1966, p. 65-73, figs. 23-25; pl. 8(12), figs. 1-10; coniferous wood; upper Tertiary; Landes, France.

PARASEQUOIA Krasilov, 1967.

Parasequoia cretacea Krasilov, 1957, p. 212-213, figs. $30 \mathrm{a}, \mathrm{b}, \mathrm{v}$; pl. 77 , figs. 1-4; stem with leaves, Coniferale; Lower Carboniferous; Southern Maritime Territory, U.S.S.R.

PARASPHENOPHYLLUM Asama, 1970.

Parasphenophyllum shansiense (Asama) Asama, 1970, p. 301-302, pl. 3, fig. 1; leaves, Sphenophyllales; Permian; China and Korea.

PARATRICHILIOXYLON Koeniguer, 1971.

Paratrichilioxylon russelli Koeniguer, 1971 , p. $160-172$, figs. $3-5$; pl. 1 , figs. $1-4$; pl. 2 , figs. 1,2 ; pl. 3 , figs. 1-4; wood, Melliaceae; Paleocene; Niger, western Africa.

PARATRIZYGIA Asama, 1970.

Paratrizygia kobonensis (Kobatake) Asama, 1970, p. 312-313; leaves, Sphenophyllales; Permian; Korea.

PARAZOLA Hall, 1969.

Parazola heterotricha Hall, 1969, p. 1175-1177, figs. 3-18, 30; fern spores; Upper Cretaceous; Choteau County, Montana, U.S.A.

PELTICUTIS Schneider, 1969.

Pelticutis amplirima Schneider, 1969, p. $15-16$, fig. 1 ; pl. 1 , figs. 4,5 ; pl. 2, fig. 1; cuticle, Myricaceae; middle Miocene; Lower Lusatia, east-central Germany.

PELTOPHOROXYLON Müller-Stoll and Mädel, 1967.
Peltophoroxylon variegatum (Ramanujam) Müller-Stoll, and Mädel, 1976 , p. 117-118; wood, Leguminosae; Miocene or Pliocene; India.

PERENNARIA Vologdin, 1962.

Perennaria ambigusa Vologdin, 1962, pt. 1, p. 251-253, fig. 51; stromatolite, Telastromataceae; lower Sinian; left bank of the Angara river, above the Ivan river, U.S.S.R.

PEREZLARIA Delevoryas and Gould, 1971.

Perezlaria oaxacensis Delevoryas and Gould, 1971, p. 616-620, figs. 1-8; ?pteridosperm fructification; Jurassic; Oaxaca, Mexico.

PERMOPADINA Ramovs, 1970.

Permopadina fallax Ramovs, 1970, $\mathbf{p}$. 97-100, fig. 1; pl. 12, figs. 1-6; algae, Dictyotales; middle Permian; eastern Karawanken, Alps, Yugoslav-Austrian border.

PERTICA Kasper and Andrews, 1972.

Pertica quadrifaria Kasper and Andrews, 1972 , p. 904-906, figs. 1-18; vascular plant, Trimerophytinae; Trout Valley Formation, Lower Devonian, northern Maine, U.S.A.

PHASMATOCYCAS Mamay, 1973.

Phasmatocycas kansana Mamay, 1973 , p. 689 , fig. $1 \mathrm{~d}-\mathrm{g}$; fertile organ, Cycadales; Lower Permian; Dickinson County, Kansas, U.S.A.

\section{PHYLLADELPHIA Bronn, 1858.}

Phylladelphia strigata Bronn, 1858, p. 133-135, pl. 7, figs. 2, 3; leaves, monocotyledon; Triassic; Raibl, Carinthia, Austria.

PHYLLOSTACHYA Khakhlov, 1964.

Phyllostachya punctata Khakhlov, p. 25-26, pl. 2, figs. 5-7; Equisetales; upper Paleozoic; northern Siberia.

PHYTOKNEME Andrews, Read and Mamay, 1971.

Phytokneme rhodona Andrews, Read and Mamay, 1971, p. 6-9, figs. 1-3; pls. 1-4; lycopod stem; Upper Devonian; Adair County, Kentucky, U.S.A.

PILBARIA Walter, 1972.

Pilbaria perplexa Walter, 1972, p. 167-170, figs. $7,51-52$; pl. 4 , fig. 4; pl. 29, figs. 2-7; stromatolite; lower Proterozoic; Western Australia.

PILICORONICUTIS Roselt and Schneider, 1969.

Pilicoronicutis velamirima Roselt and Schneider, 1969, p. 79-80, fig. 29; pl. 18, fig. 4 ; cuticle of undetermined dicotyledon; lower Oligocene; Espenhain near Leipzig, Germany.

PILIEVICUTIS Roselt and Schneider, 1969. 
Pilievicutis dentata Roselt and Schneider, 1969, p. 60-61, pl. 7, fig. 5; cuticle fragment, botanical status doubtful; middle Miocene Upper Silesia, east-central Europe.

PILIMPARICUTIS Schneider, 1969.

Pilimparicutis thompsoni (Krausel and Weyland, 1959) Schneider, 1969 , p. 23, fig. 5 ; pl. 7, figs. 1, 2; cuticle, Spotaceae; middle Miocene; Upper Lusatia, east-central Germany.

PILIPARICUTIS Roselt and Schneider, 1969.

Piliparicutis radivirgata Roselt and Schneider, 1969 , p. 73-74, pl. 16, figs. 1-4; cuticle of Lauraceae; upper Eocene; Etzdorf near Halle, East Germany.

PILISOLICUTIS Roselt and Schneider, 1969.

Pilisolicutis tenuis Roselt and Schneider, 1969 , p. 68, fig. $19 ;$ pl. 11 , figs. 1,2 ; cuticle of undetermined dicotyledon; middle Miocene, Schlacendorf near Lubbenau, East Germany.

PILOSTROMA Vologdin, 1962.

Pilostroma grumosum Vologdin, 1962 pt. 1 , p. $305-306$, fig. $69 \mathrm{~b}$; pl. 65 , figs. 1, 2, 3a ; algae, Lamellostromataceae; middle Sinian; right bank of the Nizhney Tunguska, about $34 \mathrm{~km}$ from the mouth, Turukhansk region, U.S.S.R.

PINICUTIS Schneider, 1969.

Pinicutis hexacytica Schneider, 1969, p. 28 , fig. 7 ; pl. 9, fig. 2 ; cuticle, Pinaceae; middle Miocene; near Lübbenau, Lower Lusatia, East Germany.

PLACKLESIA Bilgütay, 1968.

Placklesia multipora Bilgütay, 1968 , p. 71-74, figs. 2-6; pl. 3, figs. 1-9; calcareous algae, Thyrosoporelleae; Triassic; Packles, Hohe Wand, lower Austria.

PLAESIODICTYON Wille, 1970.

Plaesiodictyon mosellanum Wille, 1970 , p. 284-285, figs. 4-14, green algae, Chlorococcales; Keuper; Luxemburg, German border.

PLEXA Gnilovskaya, 1972.

Plexia varia Gnilovskaya, 1972, p. 121-122, fig. 53; pl. 14, fig. 3; alga, Moniliporellaceae; Upper Ordovician, eastern Kazakhstan, U.S.S.R.

PLEXOSTROMA Vologdin, 1962.

Plexostroma pleurotropum Vologdin, 1962 , pt. 1 , p. $279-280$, fig. 66 ; pl. 75 , figs. $3-5$; stromatolite, Plexostromataceae; middle Sinian; right bank of the Nizhney Tunguska, about $34 \mathrm{~km}$ from the mouth, Turukhansk region, U.S.S.R.

PLICARIZAMITES Bock, 1969.

Plicarizamites lanceolatus Bock, 1969, p. 239-242, figs. 395-401; pinnate foliage, incertae sedis; Triassic; Winterpock, Virginia, U.S.A.

PODOSTROBUS Rao and Bose, (1970) 1971.

Podostrobus rajmahalensis (Rao) Rao and Bose, (1970) 1971, p. 8384 , pl. 1, figs. 1-8; podocarpaceous male cone; Upper Jurassic; $\mathrm{Ni}$ pania, Rajmahal Hills, Bihar, India.

POIKILOPORELLA Pia, (1942) 1943.

Poikiloporella duplicata (Pia, 1920) Pia, (1942) 1943, p. 95-96; calcareous alga; Mesozoic; west of Hutkogels on the Gamsberg and other areas in the extreme western part of Austria.

POLYPODIOPTERIS Krassilov and Fedetov, 1970.

Polypodiopteris kivdensis Krassilov and Fedetov, in Fedetov, 1970, p. 96-98, pl. 15; figs. 10-17. fern, Polypodiaceae; lower Tertiary; Amur Oblast, U.S.S.R.

POMATOPHYLLUM Conti, 1947.

Pomatophyllum poerculatum Conti, 1947 , p. 53-54, fig. 3; pl. 6[2] fig. 4 ; pl. $8[4]$, fig. 5; alga, Corallinaceae; Miocene; northwestern Italy.

POMETIOXYLON Prakash and Tripathi, (1969) 1970.

Pometioxylon tomentosum Prakash and Tripathi, (1969) 1970, p. 2022, figs. $1-4$; pl. 1, figs. 1-5; wood, Sapindaceae; Tertiary; Hailakandi, Assam, India.

POODEITES Straus, 1969.

Poodeites hercynicus Straus, 1969, p. 166-169, fig. 1 ; pl. 30, fig. 2; parts of the reproductive structure of grass, Graminales; Pliocene; Willershausen, Germany.

POTAMOGETONACECARPUM Walthur, 1967.

Potamogetonacecarpum magnum Walthur, 1967, p. 264-266, fig. 3; pl. 2, figs. 4, 5; fruit remains, Potamogetonaceae; Miocene; Seifhennersdorf, Saxony, east-central Germany.

POTENTILLINA Korde, 1973.

Potentillina campanulata Korde, 1973 , p. $139-140$, fig. 37 ; pl. 17 , fig. 2; Lower Cambrian; Biryusa river, eastern Sayan, U.S.S.R.

PRAEARENARIA Vologdin, 1962.

Praearenaria bullulata Vologdin, 1962 , pt. 2 , p. 502-504, figs. 17,18 ; pl. 14, figs. 1,2 ; pl. 15 , figs. $1-4$; 
stromatolite, Trichostromaceae; Middle Cambrian; left bank of the Angara, Chadobets village, Krasnoyarskiy Kray, U.S.S.R.

PRAECHROOCOCCUS Vologdin, 1962.

Praechroococcus catervatis Vologdin, 1962 , pt. 1 , p. $162-165$, fig. 5 ; pl. 2, figs. 1-3; stromatolite, Chroococcaceae; lower Sinian; right bank of the Angara river, above the mouth of the Ivan river, southeastern Siberia, U.S.S.R.

PRESIGILLARIA Novik, 1968.

Presigillaria jongmansii Novik, 1968. p. 103-105, pl. 13, figs. 1, 2; trunk, Sigillariaceae; Lower Carboniferous; Donets basin, U.S.S.R.

PROAULOPORA Vologdin, 1962.

Proaulopora rarissima Vologdin, 1962 , pt. 2, p. 546-547, pl. 7, figs. 1-4; algae, Vologdienellea; Middle Cambrian; Kenya river, left tributary of the Abakan, environs of Torgashino village near Krasnoyarsk, U.S.S.R.

PROCHAMAESIPHON Elias, (1965) 1966.

Prochamaesiphon cumingsi Elias, (1965) 1966, p. 6-7, pl. 1, fig. 7; algae, Myxophyceae (Cyanophyta) ; Ordovician; location not given.

PROTEOKALON Scheckler and Banks, 1971.

Proteokalon petryi Scheckler and Banks, 1971, p. 874-879, figs. 130 ; progymnosperm; lower Upper Devonian; Greene County, New York, U.S.A.

PROTOCIRCOPOROXYLON Vogellehner, 1967.

Protocircoporoxylon capense (Walton, 1925) Vogellehner, 1967, p. 40-41, gymnospermous wood, Protopinaceae; Triassic; south and southwest Africa.

PROTOEPIPHYTON Vologdin, 1962.

Protoepiphyton curtofiligerum Volog. din, 1962, pt. 1, p. 248-250, fig. 50; pl. 48, figs. 1, 2; stromatolite, Telastromataceae; Lower Cambrian; western border of the Siberian platform, Turukhansk region, U.S.S.R.

PROTOGINKGOXYLON Khudayberdyev, 1971.

Protoginkgoxylon dockumense (Torrey, 1923) Khudayberdyev, in Sikstel' and others, 1971, p. 102 ; wood, Coniferales; Triassic; North America and Central Asia.

PROTOLYCOPODITES Weyland and Berer,dt, 1968.

Protolycopodites devonicus Weyland and Berendt, 1968, p. 175-177, fig.
$2 a-b$; pl. 29, figs. 18-25; leaf with sporangia, Lycopsida, Drepanophyceae; Middle Devonian; Wuppertal-Elberfeld, West Germany.

PROTOPOLYPOROXYLON Vogeilehner, 1968.

Protopolyporoxylon jurassicum (Eckhold, 1922) Vogellehner, 1968, p. 139-140; gymnospermous wood, Protopinaceae; Jurassic; Czenstochau, Poland.

PROTOSPIRA Vologdin and Strygin, 1969.

Protospira strygini Vologdin and Strygin, 1969, p. 447-448, fig. 2a, b; algae, Protospiraceae; Precambrian; Krivoy Rog, Ukranian SSR.

PROTOSEQUOIA Miki, 1969.

Protosequoia primarium (Miki) Miki, 1969, p. 731, figs. 1A, 2A, $3 \mathrm{~A}$; shoots and cones; Taxodiaceae; Tertiary; central Honshu, Japan.

PROTOTAXODIOXYLON Vogellehner, 1968.

Prototaxodioxylon choubertii (Attims, 1965) Vogellehner, 1968, p. 132-133; gymnospermous wood, Protopinaceae; Jurassic and possibly Cretaceous; Morocco, northern Africa.

PROTOTRAPA Vasil'yev, 1967.

Prototrapa douglassi Vasil'yev, 1967, p. 110-111, pl. 8, figs. 3-5; fruit, Trapaceae; Lower Cretaceous; Australia.

PSEUDAETHESOLITHON Elliott, 1970.

Pseudaethesolithon iranicus Elliott, 1970 , p. 31-35, figs. 1, 2; pls. 1-4; algae; Miocene; Tang-i-Dashtak, southwestern Iran.

PSEUDOACTINOPORELLA Conrad, 1970.

Pseudoactinoporella fragilis Conrad, 1970 , p. $66-68$, fig. 4 ; pl. 1 , figs. $1-4$; pl. 2, figs. $1-4$; pl. 8 , fig. 4 ; algae, Dasycladaceae; Lower Cretaceous; area surrounding Geneva, Switzerland.

PSEUDOANTHOS Korde, 1973.

Pseudoanthos cambricum Korde, 1973 , p. 116, fig. 3 ; pl. 4, fig. 1; algae, Pseudoanthaceae; Lower Cambrian; Kyzas river, western Sayan and Batenev ridge, U.S.S.R. PSEUDOARAUCARITES Vladimirovich, 1967.

Pseudoaraucarites gorskii Vladimirovich in Vladimirovich and others, 1967, p. 29, fig. 12; coniferous cone scale with attached seed; Lower Triassic; Pechorska depression, central Siberia, U.S.S.R. 
PSEUDOCYMOPOLIA Elliott, 1970.

Pseudocymopolia orientalis Elliott, 1970, p. 324-325, pl. 60, figs. 1, 2; algae, Dasycladaceae; Upper Jurassic to Upper Cretaceous; near Tubiti, SSE Kuching, Sarawak, Borneo.

PSEUDOGLOBATOR Grambast, 1969.

Pseudoglobator fourcasei Grambast, 1969 , p. 878,881 , pl. 4, figs. 22a28; charophyte, Clavatoraceae; Cretaceous; Ayoro, Albacte province, Spain.

PSEUDOISSINELLA Mamet and Rudloff, 1972.

Pseudoissinella alaskensis Mamet and Rudloff, 1972, p. 86, pl. 5, figs. 1719; algae, incertae sedis; Carboniferous; Itkillik Lake and Tunnel Mt., Alaska, U.S.A.

PSEUDOKAMAENA Mamet, 1972.

Pseudokamaena armstrongi Mamet, in Petryk and Mamet, 1972, $\mathrm{p}$. 779 , 780, fig. 4 ; pl. 3, fig. 12 ; algae; Korguk Formation (Viséan); Lower Carboniferous; southwestern Alberta, Canada.

PSEUDOKOMIA Rácz, (1964) 1966.

Pseudokomia cansecoensis Rácz, (1964) 1966, p. 91-92, pl. 9, figs. 5 , 6 ; pl. 10, figs. $1-3$; calcareous, red algae of uncertain affinities; the San Emiliano and Lois-Ciguera Formations, Carboniferous; Leon province, northwestern Spain.

PSEUDOLACHNOSTYLOXYLON Gottwald, 1969.

Pseudolachnostyloxylon weylandii Gottwald, 1969, p. 115-116, pl. 23, figs. 6-9, 11, 12; wood, Euphorbiaceae; Oligocene; Beja, southwestern Tunis, Tunisia.

PSEUDOLEPIDODENDROPSIS Schweitzer 1969.

Pseudolepidodendropsis carneggianum (Heer, 1871) Schweitzer, 1969 , p. 112-117, figs. 6, 7; pl. 13, figs. $4-7$; pl. 14 , figs. $1-3$; pl. 15, figs. 1-6; lycophyte, Sublepidodendraceae; Upper Devonian; Bjørn$\varnothing$ ya (Bear Island), Svalbard.

PSEUDOPIAEA Tyroff, 1966.

Pseudopiaea gigantea Tyroff, 1966, p. 256-258, figs. 1,2 ; pl. 33, figs. 4-7; pl. 34, figs. 8-11; algae; Upper Permian; Büdingen (Wetterau), West Germany.

PSEUDORHACOPTERIS Rigby, 1973.

Pseudorhacopteris ovata (McCoy, 1847) Rigby, 1973, p. 1, 2, pl. 1, fig. 1 ; pl. 2 , figs. $4-6$; pl. 3, figs. 12b-13; fernlike fronds; Upper Cretaceous; Arowa, New South Wales, Australia.
PSEUDOSTACHEOIDES Petryk and Mamet, 1972.

Pseudostacheoides loomisi Petryk and Mamet, 1972, p. 793, 795, fig. 7; pl. 9, figs. 6-8; algae; Mount Head Formation, Lower Carboniferous; southwestern Alberta, Canada.

PSEUDOTAXODIOXYLON Greguss, 1973.

Pseudotaxodioxylon jaehnicheni Greguss, 1973, p. 13, pl. 1, figs. 1, 8-12; pl. 2, fig. 1-3; pl. 3, figs. 19 ; pl. 4, figs. $1-5$; pl. 5, figs. 1-6; pl. 6 , figs. $1-7$; pl. 7 , figs. $1-4$; pl. 8, figs. 1-6; pl. 9, figs. 1-12; coniferous wood; Tertiary; central Europe.

PTERIDOCAULIS Bock, 1969.

Pteridocaulis rhombiformis Bock, 1969, p. 133-136, figs. 212-215; tree-fern, Cyatheaceae; Triassic; Winterpock, Virginia, U.S.A.

PTEROGYNOXYLON Müller-Stoll and Mädel, 1967.

Pterogynoxylon felixii (Navale) Müller-Stoll and Mädel, 1967, p. 125-126, wood, Leguminosae; Tertiary; southern India.

PUGETIA Wolfe, 1968.

Pugetia longifolia Wolfe, 1968, p. 16, fig. 7 ; pl. 2, figs. 1, 2, 4-6; leaves, Juglandaceae; lower Tertiary; King County, Washington, U.S.A.

\section{$\mathbf{R}$}

RADIOCICIELLA Dragastan, 1971.

Radiociciella subtilis Dragastan, 1971, p. 171-172, fig. 3; pl. 4, figs. $3-8$; pl. 5, figs. 1-5; algae, Dasycladaceae; Upper Jurassic and Lower Cretaceous; east Carpathians, Rumania.

RAJAHIA Kon'no, 1970.

Rajahia linggiuensis Kon'no, in Kon'no, Asama and Rajah, 1970, p. 522-527, 529-532, fig. 13, pl. 8, figs. $5-10$; pl. 9, figs. 1-6; fern Magrattiaceae; Úpper Permian; Gunong Blumut area, Johore, Malaysia.

RAMULOSTROMA Vologdin, 1962.

Ramulostroma ramulosum Vologdin, 1962 , pt. 1 , p. 286-287, fig. 60 ; pl. 66 , fig. 1 ; pl. 67 , fig. 1 ; stromatolite, Plexostromataceae; middle Sinian; right bank of the Nizhney Tunguska, about $11 \mathrm{~km}$ from the mouth, Turukhansk region, U.S.S.R.

RAMULUS Raaben, 1972.

Ramulus sociabilis Raaben, 1972, p. 40 , pl. 3 , fig. 1 ; pl. 4. figs. 2 , 4 ; pl. 25, figs. 1-4; stromatolite; 
lowermost Cambrian; Polyudova ridge, southern Timan, and the southern Urals, U.S.S.R.

REBUCHIA (Dorf, 1933) Hueber, 1970.

Rebuchia ovata (Dorf, 1933) Hueber, 1970 , a new name for Bucheria Dorf; p. 822; detached sporangia; Lower Devonian; Beartooth Butte, Wyoming, U.S.A.

RELLIMIA Leclercq and Bonamo, 1973.

Rellimia thompsonii Leclercq and Bonamo, 1973, p. 435-437, fig. 1; an anagram for Milleria (Propteridium) thompsonii Lang Emend. Leclercq and Bonamo, 1971, sporangial branches, progymnosperm; Devonian; Scotland.

RENIERA Stockmans, 1968.

Reniera verrucosa Stockmand, 1968, p. 11-12, pl. 3, figs. 5-5a; thallophyte; Middle Devonian; Brabant Massif region, central Belgium.

RETICUTIS Roselt and Schneider 1969.

Reticutis communis Roselt and Schneider, 1969 , p. 61 , pl. 10, fig. 3 ; cuticle of upper side of a leaf; Miocene; Lower Lusatia and Upper Lusatian highlands, Germany.

RHAMNOXYLON Chitaley and Kate, 1972.

Rhamnoxylon intertrappea Chitaley and Kate, 1972, p. 43-33, pl. 1, figs. 1-6; wood, Rhamnaceae; uppermost? Cretaceous; Mohgaonkalan, Chhindwara District, Madhya Pradesh, India.

RHAPHIDOPTERIS Barale, 1972, a new name for Stenopteris Saporta, 1872.

Rhaphidopteris astartensis (Harris, 1932) Barale, 1972, p. 1011-1014; filiform foliage; Upper Triassic; Scoresby Sund, East Greenland.

RHETINOTHECA Leisman and Peters, 1970.

Rhetinotheca tetrasolenata Leisman and Peters, 1970, p. 871-873, figs. 1-16; pteridosperm male fructification; Middle Pennsylvanian; Sahara coal mine, Williamson County, Illinois, U.S.A.

RHYNCHOSPERMA Taylor and Eggert, 1967.

Rhynechosperma quinnii Taylor and Eggert, 1967, p. 984-991, figs. 125, petrified seed; Upper Mississippian; Washington County, Arkansas, U.S.A.

RIBOIDOXYLON Page, 1970.

Riboidnxylon cretaceae Page, 1970 , p. 1141,1143 , figs. $7,9-11$; stem of angiospermous wood; Upper Cretaceous; Stanislaus County, central California, U.S.A.

RIGBYOCARPUS Tidwell, 1967.

Rigbyocarpus ebracteatus Tidwell, 1967 , p. 57 , pl. 10 , figs. 3,7 ; table 6; seed; Lower Pennsylvanian; Utah, U.S.A.

RIMILATERICUTIS Roselt and Schneider, 1969.

Rimilatericutis tenuis Roselt and Schneider, 1969, p. 81-82, fig. 31; pl. 19, fig. 2; cuticle, probably Dioscoraceae; lower Miocene; Wiesa near Kamenz, Upper Lusatia, East Germany.

RISSIKIA Townrow, 1967.

Rissikia media (Tenison Woods, 1883) Townrow, 1967, p. 103-111, $115,117,121$, pl. 1a-b, e-h; pl. 2a-c, f, h, i; leafy shoots, pollen and seed cones, Podocarpaceae; Upper Triassic; Queensland, Australia.

ROBINOXYLON Müller-Stoll und Mädel, 1967.

Robinoxylon zirkelii (Platen) Müller-Stoll and Mädel, 1967, p. 145147, pl. 38, figs. 90,91 ; pl. 39, fig. 92 ; fig. 13 ; wood, Leguminosae; Niobrara River, northern Nebraska, U.S.A.

ROSENKRANTZIA Koch, 1972.

Rosenkrantizia picrodendroides Koch, 1972, p. 7-18, 20, figs. 2-5, 11 ; pls. 1-18; dicotyledonous fruit; Upper Cretaceous; Nûgssuaq, West Greenland.

ROSTOPORELLA Segonzac, 1970.

Rostoporella oviformis Segonzac, 1970 , p. 339 , pl. 20 , fig. 19 ; algae Acetbulariacees; lower Tertiary; southern France.

ROTUNDOLEPIS Bock, 1969.

Rotundolepis intermedia Bock, 1969, p. 294-298, figs. 506-515; coniferous foliage (?Voltziales) ; Triassic; Carversville, Pennsylvania, U.S.A.

RUNCARIA Stockmans, 1968.

Runcaria heinzelinii Stockmans, 1968 , p. 35-36, pl. 7, figs. 5-9b; possibly a pteridospermaceous seed, compared with Condrusia; Middle Devonian; Brabant Massif region, central Belgium.

RUSSELLITES Mamay, 1968.

Russellites taeniata (Darrah) Mamay, 1968, p. I-9, pls. 1-3; cycadlike fronds; Lower Permian; Baylor County, Texas, U.S.A.

\section{$\mathrm{S}$}

SACHALINIA Vakhrameev, 1968.

Sachalinia sachalinensis Vakhrameev, 1968, p. 10-11, figs. 3, 4; 
pl. 2, figs. 1,2 ; pl. 3 , figs. 1,2 ; pl. 4, figs. 3, 4; fern; Upper Cretaceous; Tatarsk depression Sakhalin, U.S.S.R.

SAJAKIA Senkevich, 1961.

Sajakia rhomoidea Senkevich, 1961, p. 180-181, fig. 10, pl. 31, figs. 3-5; lycopod, Leptophloeaceae; Upper Devonian; northeastern Pribalkhash, Kazakhstan, U.S.S.R. SAJANIA Vologdin, 1962.

Sajania frondosa Vologdin, 1962, pt. 2, p. $482-483$, pl. 5, fig. 4; algae, Sajaniaceae; Middle Cambrian; northwestern branch of the eastern Sayan Mountains, central Siberia, U.S.S.R.

SAKKIONELLA Segonzac, 1970.

Sakkionella avallanensis Segonzac, 1970 , p. $1881-1882$, figs. 1,2 ; reproductive organ, Dasycladales; upper Paleocene; Pyrénées, Ariège, France.

SAROSIELLA Segonzac, 1972.

Sarosiella fermollis Segonzac, 1972, p. 394-396, pl. 1, figs. 1-6; alga; Paleocene; The Pyrénées, HauteGaronne, Aude and Ariège, southern France.

SATSANGIA Srivastava and Maheshwari, 1973.

Satsangia campanulata Srivastava and Maheshwari, 1973, p. 222227, figs. $1-3$; pl. 1, figs. 1-6; ?fructification incertae sedis; Triassic; Nidpuri, Sidhi district, Madhya Pradesh, India.

SWADONIA Hueber, 1971.

Swadonia ornata (Dawson, 1871) Hueber, 1971b, p. 641-642; a new name for Psilophyton princeps var. ornatum; stems, Zosterophyllaceae; Devonian; Gaspé bay, Canada.

SAXONICUTIS Roselt and Schneider, 1969.

Saxonicutis ancoriterminata Roselt and Schneider, 1969, p. 72-73, fig. $7 \mathrm{a}$; pl. 17, figs. 1, 2; cuticle of undetermined dicotyledon; Wetro, Upper Lusatia, East Germany.

SCANDOPHYCUS Vologdin, 1962.

Scandophycus crisptobilis Vologdin, 1962 , pt. 1 , p. 296-298, figs, $76 \mathrm{~b}$, 77, 78, pl. 71, figs. 1, 2a; pl. 72, figs. 1-3; stromatolite, Scandophycaceae; middle Sinian; right bank of the Nizhney Tunkuska, Turukhansk region, U.S.S.R., and Algeria, northwestern Africa.

SCHUGURIA Chirkova-Zalesskaya, 1959.

Schugiria ornata Chirkova-Zalesskaya, 1959: p. 92-95, figs. 88-95; pl. 11, figs. 58-59; pl. 18, figs. 101-106; pl. 19, figs. 107-110; pl. 20, figs. 111-112; pl. 21, figs. 113114 ; pl. 22 , figs. $115-117$; pl. 23 , figs. 119-121; pl. 32, fig. 180; cuticle, incertae sedis; Devonian; Ural-Volga area, U.S.S.R.

SCIADOPHY TOPSIS Ishchenko, 1968.

Sciadophytopsis triassicum Ishchenko, in Karandievskiy, Ishchenko and Kir'yamnov, 1968, p. 90-93, fig. $12 \mathrm{a}$, b; pl. 14, figs. 6-8; pl. 15 , figs. $1-5$; pl. 16, fig. 1 ; rosettelike plant and sporangia, Sciadophytaceae; Lower Devonian; left bank of the Dnestra, Volyn-Podolia, U.S.S.R.

SCIADOPITOPHYLLUM

Christophel, 1973.

Sciadopitophyllum canadense Christophel, 1973, p. 61-65, figs. 2-10; compression of conifer shoots and leaves; Upper Cretaceous to Paleogerie; Smokey Tower location, western Alberta, Canada.

SCIADOPITYCUTIS Schneider, 1969.

Sciadopitycutis marcodurensis (Ermelling, 1955) Schneider, 1969, p. 26, 27, pl. 8, fig. 6; cuticle, Sciadopityaceae; middle Miocene; Hosena near Senftenberg, Lower Lusatia, east-central Germany.

SCOTOXYLON Vogellehner, 1968.

Scotoxylon hornei (Seward and Bancroft, 1913) Vogellehner, 1968, p. 150-151; gymnospermous wood, Protopinaceae; Upper Jurassic; Helmsdale, Sutherland, Scotland.

SELESICUTIS Roselt and Schneider, 1969.

Selesicutis prosenchymatica Roselt and Schneider, 1969 , p. 80-81, fig. 30 ; pl. 20 , fig. 1 ; cuticle, incertae sedis; upper Lower Carboniferous; Upper Silesia, east-central Europe.

SEPTOMEDULLOPITYS Lepekhina, 1969.

Septomedullopitys sibirica Lepekhina, 1969 , p. 137-139, pl. 5, fig. 6 ; pl. 6, figs. 1-7; wood with xylem; Upper Permian; Kuznets basin, Kemerova Oblast, U.S.S.R. SERLIGIA Korde, 1973.

Serligia fragilis Korde, 1973, p. 143-145, figs. 13,14 ; pl. 20, figs. $2-4$; pl. 21, figs. $1-5$; algae, Serligiaceae; Lower Cambrian; Ser. lig river, eastern Tannu-Ola, U.S.S.R.

SERMAYA Eggert and Delevoryas, 1967.

Sermaya biseriata Eggert and Delevoryas, 1967 , p. $172-178$, pls. $34-$ 
38; fern, Sermayaceae; Upper Pennsylvanian; Berryville, Illinois, U.S.A.

SERTOSTROBUS Grauvogel-Stamm, 1969.

Sertostrobus laxux GrauvogelStamm, 1969, p. 112-116, figs. 11, 12,13 ; pl. 2, figs. $9-18$; coniferous male cone; Lower Triassic; Vosges, Lorraine, eastern France.

SESSAOXYLON Koeniguer, 1971.

Sessaoxylon paleocenicum Koeniguer, 1971, p. 313-320, figs. 5-8; pl. 2, figs. 1-4; wood, incertae sedis; Paleocene; Krebb de Sessao, Niger, Africa.

SEWARDIOXYLON Gupta, 1971.

Sewardioxylon sahnii Gupta, 1971, p. $160-165$, figs. 1,2 ; pls. 35,36 , figs. 1-19; cycadean wood, Cycadopsida; Jurassic; Rajmahal Hills, Bihar, India.

SGROSSOELLA De Castro, 1969.

Sgrossoella parthenopeia De Castro, 1969 , p. 90-102, 112-153, figs. 1, 4-6; pls. 1-20, algae, Chlorophyceae; Upper Cretaceous; Campania, southern Italy.

SHANGANELLA Vologdin, 1969.

Shanganella tuvaica Vologdin, 1969, p. 1377-1378, figs. 1, 3a, v, b; blue-green algae, Shanganellaceae; Lower Cambrian; UlugShangan canyon, Tuva, southern Siberia, U.S.S.R.

SIAMPORIDIUM Endô, 1969.

Siamporidium elongatum Endô, 1969 , p. 39-40, fig. 1 ; pl. 5, fig. 5; pl. 6 , figs. 1,2 ; pl. 7, figs. $1-3$; algae, Corallinaceae; Permian; Thailand.

SIDERINIUM Prakash and Awasthi, (1969) 1970.

Siderinium demaliense Prakash and Awasthi, (1969) 1970, p. 40-41, pl. 6, figs. 33-37; silicified wood, compared with modern wood, incertae sedis; Tertiary; Namsang river bed near Deomali, Assam, India.

SILICOPHYLLUM Weyland, Kilpper and Berendt, 1967.

Siliciphyllum heerlense, Weyland, Kilpper and Berendt, 1967, p. 163-166, figs. 23-30; pl. 31, figs. $39-42$; pl. 32, figs. 43-47; dicotyledonous leaves; Miocene; Heerlen, Limburg province, southeast Netherlands.

SINUATOPORELLA Gnilovskaya, 1972.

Sinuatoporella bucera Gnilovskaya, 1972, p. 93-96, fig. 42; pl. 9, figs. $1,2,4$; alga, Codiaceae; Úpper
Ordovician; eastern Kazakhstan, U.S.S.R.

SLIVKOVIA Meyen, 1969.

Slivkovia petschorensis Meyen, 1969, p. 97-100, figs. 5, 6; pl. 15, figs. 1-13; shoots and foliage, incertae sedis; Permian; Pechoria, Cisurals, U.S.S.R.

SOGDIANIA Burakova, 1971.

Sogdiania abdita Burakova, 1971, p. $3-7$, pl., figs. $1 \mathrm{a}, 1 \mathrm{~b}, 1 \mathrm{v}, 1 \mathrm{~g}$; ?reproductive structure, incertae sedis; Middle Jurassic; near Yaksho-Saydun village, left bank of the Obi-Niou river, western Siberia, U.S.S.R.

SONNERATIORHIZOS Chitaley (1968) 1969.

Sonneratiorhizos raoi Chitaley (1968) 1969, p. 244-246, figs. 18 ; pl. 1 , figs. 1-6; root, dicotyledon; probably Paleocene; Mongaonkalan, Chhindwara district, Madhya Pradesh, India.

SPHAEROCARYA Dorofeev, 1970.

Sphaerocarya uralensis Dorofeev, 1970 , p. 36-38, fig. 3; pl. 6, figs. 1-10; endocarp, Juglandaceae; Miocene; middle Urals, Visim district, Sverdlovsk region, U.S.S.R.

SPHAEROPORELLA Antropov, 1967.

Sphaeroporella aksubaica Antropov, 1967, p. 122-123, pl. 27, figs. 1-3; algae, Siphonales; Lower Carboniferous; central part of the east Russian platform, U.S.S.R.

SPHAEROTHALLUS Vologdin, 1962.

Sphaerothallus spissus Vologdin, 1962 , pt. 1, p. 298-300, fig. 79; pl. 73, figs. 1-3; stromatolite. Scandophycaceae; middle Sinian; right bank of the Nizhney Tunguska, about $11 \mathrm{~km}$ from the mouth, Turukhansk region, U.S.S.R.

SPINCTOPORELLA Mamet and Rudloff, 1972.

Spinctoporella lisburnensis Mamet and Rudloff, 1972, p. 84, pl. 4, figs. 1-6; algae. Dasycladaceae; Carboniferous; Itkillik and Shainin Lakes, Alaska, U.S.A.

SPINOPALMOXYLON Weyland, Kilpper and Berendt, 1966.

Spinopalmoxylon daemonorops (Unger, 1860) Weyland, Kilpper and Berendt, 1966, p. 88; spine-bearing palm, Arecaceae; Tertiary; Wetterau, West Germany.

SPORINULA Korde, 1973.

Sporinula palmata Korde, 1973, p. $149-150$, pl. 25, fig. 2; algae, Tomentulaceae; Lower Cambrian; 
Bazaikha river, eastern Sayan, U.S.S.R.

SQUAMOPHYLLUM G. P. Radchenko, 1934.

Squamophyllum

actaeonelloides

(Geinitz, 1871) G. P. Radchenko, 1934 , p. 35,37 , pl. 12 , figs. 9-12; scaly cordaitean leaves; Permian; Kuznetsk basin; south-central Siberia, U.S.S.R.

STACHEIA Brady, 1876.

Stacheia marginuloides Brady, 1876, Petryk and Mamet, 1972, p. 784, calcareous algae (originally described by Brady as a foraminifer); Carboniferous; England and Scotland.

STACHEOIDES Cummings, 1955.

Stacheoides palytrematoides (Brady, 1876), Petryk and Mamet, 1972, p. 785 ; calcareous algae (originally described by Brady and later by Cummings as Foramanifera); Carboniferous; England and Scotland.

STEGANOTHECA Edwards, 1970.

Steganotheca striata Edwards, 1970 , p. $451-454$, fig. 1 ; pl. 84 , figs. $1-$ 6 ; pl. 85, figs. 1-8; branching axes with terminal sporangia, Rhyniaceae; Lower Devonian, South Wales, Great Britain.

STOLBERGIA Fairon, 1967.

Stolbergia spiralis Fairon, 1967, p. 23-24, pl. 1, figs. $1-4$; pl. 2, figs. $5-12$; pl. 3 , figs. $13,15-16,19$ 21 ; pl. 4, figs. 22-27; axis, incertae sedis (?psilophyte); Middle Devonian; Stolberg, West Germany.

STRIATOTAXUS Bock, 1969.

Striatotaxus longifolia (Emmons) Bock, 1969, p. 335-338, figs. 575582; for Walchia longifolius Emmons, 1857) coniferous foliage (?Taxales); Triassic; Carversville, Pennsylvania, U.S.A.

STRIATATHALLUS Krasilov, 1973.

Striatothallus adnicanicus Krasilov, 1973, p. 98-99, pl. 44, figs. 33-44; pl. 45, figs. 45-57; bryophyte, Hepaticae; Early Cretaceous; Bureya basin, near the mouth of the Adnican river, U.S.S.R.

SUERIA Menendez, 1965.

Sueri rectinervis Menendez, 1965 , p. 75-79, pls. 1-4; leaves and stoma, Cycadales; lowermost Cretaceous; Ticó, Santa Cruz province, Argentina.

SUJFUNOPHYLLUM Krasilov, 1967.

Sujfunophyllum dichotomum Krasilov, 1967 , p. 227,230 , fig. $30 \mathrm{zh}$; pl. 85, figs. 1-7; leaves Gymno- spermae, incertae sedis; Lower Cretaceous; southern Maritime Territory, U.S.S.R.

SULCOCLADUS Stockmans, 1968

Sulcocladus multipunctatus Stockmans, 1968 , p. 32 , 33, pl. 13, figs. 1-4; similar to calamophyton (calamarian?), incertae sedis; Middle Devonian; Brabant Massif region, central Belgium.

SUPPILULIMAELLA Elliott, 1968.

Suppilulimaella polyreme Elliott, 1968 , p. 495-496, pl. 95, figs. 1-4; algae, Dasycladaceae; Lower Cretaceous, Turkey.

SURANGEA Chitaley and Sheikh, (1971) 1972.

Surangea mohgaoense Chitaley and Sheikh, (1971) 1972, p. 123-126, figs. $1-4$; pl. 1 , figs. $1-8$; a pteridophytic fructification, incertae sedis; Paleocene; Mohgaonkalan, Madhya Pradesh, India.

SVALBARDOXYLON Vogellehner, 1968.

Svalbardoxylon johnsonii (Schroter, in Heer, 1880) Vogellehner, 1968, p. 152-154; gymnospermous wood, Protopinaceae; Cape Dufferin, Spitsbergen.

SWINTONIOXYLON Prakash and Tripathi, 1968.

Swintonioxylon hailkandiense Prakash and Tripathi, 1968 , p. 115116, figs. 3, 4; wood, compared with the modern wood Swintonia floribunda; Tertiary; Hailakanda, Cachar district, Assam, India.

\section{$\mathbf{T}$}

TAENIOPHYTON Weyland and Berendt, 1968.

Taeniophyton inopinatum Weyland and Berendt, 1968, p. 172-174, fig. 1 ; pl. 27, figs. $1-9$; pl. 28, figs. 10-14; compared with Taeniocrada, ?Rhyniaceae; Middle Devonian; Wuppertal-Elberfeld, West Germany.

TANINIA Korde, 1973.

Taninia tomentosa Korde, 1973, p. $150-151$, pl. 25, fig. 3 ; pl. 26 , fig. 1; algae, Tomentulaceae; Lower Cambrian; Bazaikha River, eastern Sayan, Kuznetskiy Alatau, Sahashtykgol river, Western Sayan, U.S.S.R.

TANTALLOSPERMA Barnard and Long, 1973.

Tantallosperma setigera Barnard and Long, 1973, p. 99-103, fig. 5; pl. 2 , fig. 21 ; pl. 4 , figs. $30-40$; seed, closely associated with the stem Buteoxylon gordonianum but its relationship has not been es- 
tablished; Upper Carboniferous; Oxroad Bay, East Lothian, Scotland.

TARIOUFETIA Bertrand-Sarfati, 1972.

Tarioufetia hemispherica BertrandSarfati, 1972, p. 56-60, figs. 13, 16,17 ; stromatolite; upper Precambrian; Mauritania, Sahara Occidental.

TATARINA Meyen, 1969.

Tatarina olferievii Meyen, 1969, $\mathrm{p}$. 99-100, pl. 4, figs, 1-9; leaf epidermis, incertae sedis; Upper Permian; right bank of Vokhma river, right tributary of Vetluga river, Kirovskaya Oblast, U.S.S.R.

TAXOXYLUM Unger, 1842.

Taxoxylum aykii (Göppert, 1841, non Ad. Brong.) Unger, 1842, p. 33. See also Unger, in Endlicher, 1847 , p. 308. Later spelling: Taxoxylon aykei, Unger, $1850, p$. 390 ; coniferous wood; Miocene; Hungary.

TCHAUNIA Samy'lina and Filippova, 1970.

Tchaunia tchaunensis Samy'lina and Filippova, 1970, p. 92-94, fig. 3; pl. 12 , figs. $1-5$; fern, ?Dicksoniaceae; Cretaceous; northeastern U.S.S.R.

TEDELEA Eggert and Taylor, 1966.

Tedelea glabra (Baxter) Eggert and Taylor, 1966, p. 54-70, figs. 1-8; pls. 8-11; fern, Tedeleaceae; Middle Pennsylvanian; Illinois, Indiana, and Kansas, U.S.A.

TELANGIOPSIS Eggert and Taylor, 1971.

Telangiopsis arkansanum Eggert and Taylor, 1971, p. 30-37, figs. $1-8$; compressed synangiate pollen organ; Upper Mississippian; Lake Lincoln damsite, Washington County, Arkansas, U.S.A.

TELASTROMA Vologdin, 1962.

Telastroma tenuirimulatum, Vologdin, 1962, pt. 1, p. 241-244, pl. 45, figs. $1-3$; stromatolite, Telastromataceae; Sinian; region of the lower Nizhney Tunguska, left bank, Durnoy cape, Turukhansk, U.S.S.R.

TETRAMELEOXYLON Lakhnapal and Verma, (1965) 1966.

Tetrameleoxylon prenudiflora Lakhnapal and Verma, (1965) 1966, p. 209-212 figs. 1, 2; dicotyledonous wood; Tertiary (probably Lower Eocene); Mohgaonkalan, Madhya Pradesh, India.

TETRAPLEUROXYLON MüllerStoll and Mädel, 1967.

Tetrapleuroxylon ingaeforme (Fe- lix) Müller-Stoll and Mädel, 1967, p. 113-114, fig. 4 ; pl. 28, figs. 20 , 21 ; pl. 29 , fig. 26 ; wood, Leguminosae; Tertiary; Brazil.

TEXTURATA Gnilovskaya, 1972.

Texturata tubulosa Gnilovskaya, 1972, p. 122-124, pl. 14, fig. 1; alga, Moniliporellaceae; Upper Ordovician; central Kazakhstan, U.S.S.R.

THAILANDOPORELLA Endô, 1969.

Thailandoporella phlongphrabensis Endô, 1969, p. 66-67, fig. 3; pl. 22 , figs. 8-9; pl. 25, figs. 2, 3 ; algae, Dasycladaceae; Permian; Thailand.

THAINGUYENOPTERIS Srebrodolskaya, 1969.

Thainguyenopteris parvipinnulata Srebrodolskaya, 1969 , p. 87-98, figs. $1-3$; pl. 1, figs. 1-6; pl. 2, figs 1 , 1a, 1b; foliage, Filicinae, incertae sedis; Upper Triassic; northern Viet Nam.

THAMNOCLADITES Stockmans, 1968.

Thamnocladites vanopdenboschii Stockmans, 1968 , p. 39-40, pl. 1, figs. $1-7$; pl. 14 , fig. 8 ; axes, incertae sedis; Middle Devonian; Brabant Massif region, central Belgium.

THARMA Wray, 1967.

Tharma glauea Wray, 1967, p. 1921, fig. 7 ; pl. 4, figs. $1-5$; aldae, incertae sedis; Upper Devonian; Canning basin, Western Australia.

THUJADENDRON Bock, 1969.

Thujadendron pristinum Bock, 1969, p. 352-356, figs. 619-626; coniferous fragments (?Cupressaceae); Triassic; Turners Falls, Massachusetts, U.S.A.

THUJATOSTROBUS Bock, 1969.

Thujatostrobus triassicus Bock, 1969 , p. $350-352$, figs. 615-618; coniferous cone scales (?Cupressaceae) ; Triassic; Gwynedd, Pennsylvania, U.S.A.

THURINGICUTIS Roselt and Schneider, 1969.

Thuringicutis Tenurimata Roselt and Schneider, 1969. p. 70, fig. 21; pl. 14, figs. 1-4; cuticle of undetermined dicotyledon; upper Eocene; Profen near Seitz, East Germany.

TIMANELLA Vologdin, 1969.

Timanella gigas Vologdin; $1969, \mathrm{p}$. $672-675,4$ figs., siphon algae, Timanollaceae; upper Proterozoic; western zone of the Timan mountain range, U.S.S.R. 
TINSLEYA Mamay, 1966.

Tinsleya texana Mamay, 1966, p. E10-E11, figs. 1, 2; pls. 1-3; fronds with laminally borne seeds, Callipteridaea; Lower Permian; Baylor County, Texas, U.S.A.

TIRASISIA Ischenko, 1968.

Tirassia incisa Ischenko in Karandievskiy, Ischenko and Kir'yanov, 1968, p. 105-106, pl. 22, figs. 4-8, reproductive organ, incertae sedis; Lower Devonian; right and left; banks of the Dnestra, VolynPodolia, U.S.S.R.

TOLLIA Sveshnikova and Budantsev, 1969.

Tollic, cunninghamioides Sveshnikov'a and Budantsev, 1969, p. 8485 , pl. 32 , figs. $3-49$; pl. 33 , figs. 4-2:2; leaves, Taxodiaceae; Upper Cretaceous; Derevyannye Mountain; New Siberian Islands, U.S.S.R.

TOMENTULA Korde, 1973.

Tomentula villosa Korde, 1973, p. 147-148, fig. 8; pl. 22, fig. 4 ; pl. 23, fig. 1; algae, Tomentulaceae; Lower Cambrian; Bazaikha river, eastern Sayan, U.S.S.R.

TORE TZIA Stanislavsky, 1973.

Toretzia angustifolia Stanislavsky, 1973 , p. 91, 93-94, fig. 1a-g; pl. 12 , figs. 1-3; branch bearing shoots with megastrobili, Ginkgoales, Toretziaceae; Upper Triassic; Raysko hamlet, Kazennyy Torets river, northwestern outskirts of the Donets basin, U.S.S.R.

TORTKOPHYTON Yurina, 1969.

Tortkophyton globosum Yurina, 1969 , p. $72-73$, fig. 35 ; pl. 29, figs. 1-4; zygopterid fructification; Devonian; central Kazakhstan, U.S.S.R.

IRIADOSTROBUS Bock, 1969.

Triadostrobus fenestraeformis Bock, 1969 , p. $330-331$, figs. $567-572$; cones, incertae sedis; Triassic; Winterpock, Virginia, U.S.A.

TRIASOCOCOLADUS Archangelsky, 1966.

Triasococlandus tigrensis Archangelsky, 1966, p. 276-280, figs. 21, 25 ; pl. 4 , fig. 21 ; pl. 5 , figs. $22-$ 39 ; pl. 8, figs. 56-67; air bladders, gymnospermous leaves, Podocarpaceae; Lower Cretaceous; Santa Cruz province, Argentina.

TRIASSIFLORITES Bock. 1969.

Triassiflorites grandiflora Bock, 1969 , p. 265-275, figs. 442-462; fruiting body, incertae sedis; Triassic; Winterpock, Virginia, U.S.A.
TRICHOSTROMA Vologdin, 1962.

Trichostroma capilliforme Vologdin, 1962 , pt. 1, p. 253-255, fig. 52; pl. 49 , figs. $1-4$; stromatolite, Trichostromataceae; Sinian; right bank of the lower Nizhney Tunguska, Durnoy cape, Turukhansk region, U.S.S.R.

TRICLYPELLA Grambast, 1969.

Triclypella calcitrapa Grambast, 1969, p. 878, 881, pl. 1, figs. 1a-7; charophyte, Clavatoraceae; Cretaceous; Castellon province, Spain.

TRICOSTIUM Krasilov, 1973.

Tricostium papillosum Krasilov, 1973 , p. $100-101$, pl. 48 , figs. $75-$ 86; stem fragments and serrate leaves, bryophyte, Musci; Late Jurassic; Bureya basin, near the mouth of the Umalta river, U.S.S.R.

TRILOBOXYLON Matten and Banks, 1966.

Triloboxylon ashlandicum Matten and Banks, 1966, p. 1020-1026, figs. 1-18; pteridopsid axes; Upper Devonian; northern Catskills, New York State, U.S.A.

TRYASOTAENIA Gniloviskaya, 1971.

Tryasotaenia podolica Gnilovskaya, 1971, p. 106-107, pl. 11, figs. 1-5; algae, Vendotaenides; upper Precambrian; Dniester region of Podolia and Moldavia, U.S.S.R.

TUBERCULARIA Vologdin, 1962.

Tubercularia latiuscula Vologdin, 1962 , pt. 2, p. 488-489, fig. 7; pl. 7, figs. 1a, 2a; stromatolite, Scandophyceae; upper Lower Cambrian; Yanguda river basin, U.S.S.R.

TUBERICUTIS Roselt and Schneider, 1969.

Tubericutis hemisphaeria Roselt and Schneider, 1969, p. 74-75, figs. 24, 25; pl. 18, figs. 1-3; cuticle of undetermined dicotyledon: upper Eocene; Lochau near Halle, East Germany.

TUBOMORPHYTON Korde, 1973.

Tubomorphyton botomense (Epiphyton botomense Korde, 1955) Korde, 1973, p. 204-205, algae, Epiphytaceae; Lower Cambrian; Lena river, Botoma, Mukhatta, Yakutsk, U.S.S.R.

TUBULISTROMA Vologdin, 1962.

Tubulistroma scrofulosum Vologdin, 1962 , pt. 1 ; p. $310-312$, fig. 89; pl. 77, fig. 1 ; algae, Porostromataceae; upper Sinian; Borlog river, Burul'deyka river system, southwestern Cisbaikal, U.S.S.R.

TUMIDOPHYTON Vologdin, 1962.

Tumidophyton nucamentum Volog- 
din, 1962, pt. 2, p. 494-495, fig. 11; pl. 11, fig. 3; stromatolite, Tumidophyceae; Lower Cambrian; area of the upper course of the Lena, Irkutsk, Oblast, U.S.S.R.

TUNGUSSOCARPUS Sukhov, 1969.

Tungussocarpus tychtensis (Zalessky, 1937) Sukhov, 1969, p. 163-166, pl. 26, figs. 1-7; gymnosperm seed; Permian; Kuznets and Tunguska basins, central Siberia, U.S.S.R.

TUNGUSSOPTERIS Vladimirovich, 1967.

Tungussopteris sphenopteroides Vladimirovich in Vladimirovich and others, 1967 , p. 24-25, fig. 3 ; fern; Lower Triassic; Tunguska basin, central Siberia, U.S.S.R.

TUNIKATA Krylov, 1969.

Tunikata nochtuica Krylov, in Krylov, Korolyuk and Siderov, 1969, p. 210-212, figs, 58-60; pl. 42, figs. 1-4; pl. 43, figs. 1, 2; stromatolite; Precambrian; near Nokhtuyysk village, Yakutsk Oblast, northeastern Siberia, U.S.S.R.

TURKESTANIOXYLON Khudayberdyev, 1971.

Turkestanioxylon metasequoianum Khudayberdyev, in Khudayberdyev, Gomolitskii and Lobanova, 1971, p. 37-40, pl. 27, figs. 1-4; wood, Taxodiaceae; Upper Cretaceous; northern slope of Turkestan range, Uzbekskoy SSR.

TUZHYKOVIELLA Vladimirovich,

Tuzhykoviella elegans Vladimirovich, 1972, p. 96-97, pl. 2, fig. 2; fernlike foliage; Middle Triassic; Orenburg, Ural region, U.S.S.R.

TYPHAEPHYLLUM Prakash and Boureau, 1970.

Typhaephyllum scammoni Prakash and Boureau, 1970, p. 94-95, 101105, fig. 11 , pls. $1-5$, figs. $1-10$; herbaceous dicotyledon, Typhaceae; upper Miocene; Vantage, Kittitas County, Washington, U.S.A.

\section{U}

UMALTOLEPSIS Krasilov, 1972.

Umaltolepsis vachrameevii Krasilov, 1972 , p. 62-64, fig. 10n-p, f; pl. 21 , fig. $5 a$; pl. 22, figs. 5-8; pl. 23, figs. $1,2,5-7,13$; ginkgophyte seed, Pseudotorelliaceae; Upper Jurassic; right bank of the Bureya river, Uman'tinski section, Khavarosk Kray, U.S.S.R.
UMBELLULA Korde, 1973.

Umbellula minuta Korde, 1973, p. 135 , pl. 15, fig. 3 ; algae, Cambrinacaeae; Lower Cambrian; Bazaikha river, eastern Sayan, U.S.S.R.

URALIA Chirkova-Zalesskaya, 1959.

Uralia bella Chirkova-Zalesskaya, 1959 , p. $86-88$, figs. $77-79$; pl. 6 , figs. $29-31$; pl. 25 , figs. 125,126 ; sporangia, ?Trimerophytina; Devonian; Ural-Volga area, U.S.S.R.

UTERIOIDES Segonzac, 1970.

Uterioides copiosus Segonzac, 1970, p. $1883-1884$, fig. $3 a-4$; reproductive organ, Dasycladales; upper Paleocene; Pyrenees.

\section{V}

VALMEYERODENDRON Jennings, 1972.

Valmeyerodendron triangularifolium Jennings, 1972 , p. $74-80$, fig. 3; pls. 17-21, figs. 1-45; lycopod stems, cuticle and leaves; affinities with Sublepidodendraceae close; Trout Hollow, Monroe County, Illinois, U.S.A.

VARIPILICUTIS Schneider, 1969.

Varipilicutis liblarensis (Krausel and Weyland, 1954) Schneider, 1969 , p. 27, 28, fig. 6 ; pl. 9, fig. 1; cuticle. Discoreaceae; upper Oligocene; Liblar, West Germany.

VENDOTAENIA Gnilovskaya, 1971.

Vendotaenia antiqua Gnilovskaya, 1971 , p. 105-106, pl. 11, figs. 6-8; algae, Vendotaenides; upper Precambrian; Leningrad province, Dniester region of Podolia, U.S.S.R.

VERSIPALMICUTIS Schneider, 1969.

Versipalmicutis undulata Schneider, 1969, p. 28-29, pl. 9, figs. 3, 4; cuticle, Palmae; middle Miocene; Tagebau Spreetal, Lower Lusatia, east-central Germany.

VERTICILIAPHYTON Baxter, 1967.

Verticiliaphyton paradoxum Baxter, 1967 , p. 71-76, figs. 1-17: woody axis, incertae sedis; Middle Pennsylvanian; Oskaloosa, Iowa, U.S.A.

VESICOPHYTON Vologdin and Drozdova, 1969.

Vesicophyton punctatum Vologdin and Drozdova, 1969, p. 1419-1421, fig. I:1-6; microscopic colonial algae, Gloeocapsaceae; Precambrian; Batenev ridge, Krasnoyarskiy Kray, U.S.S.R. 
VESICULARIA Vologdin 1962.

Vesicularia nidifica Vologdin, 1962, pt. 1, p. 226-228, fig. 36 ; pl. 39 , figs. 1, 2; stromatolite, Vesiculariaceae; upper Sinian; Borlog river, tributary of Burul'deyka river, southwest of Cisbaikal, U.S.S.R.

VILLOSOPORELLA Gnilovskaya, 1972.

Villosoporella villosa Gnilovskaya, 1972 , p. 124-126, fig. 54; pl. 14, fig. 2; alga, Moniliporellaceae; Upper Ordovician; eastern Kazakhstan, U.S.S.R.

VINEA Wolfe, 1968.

Vinea pugetensis Wolfe, 1968, p. 14-15, fig. 4; pl. 2, fig. 3; leaves, Juglandaceae; lower Tertiary; King County, Washington, U.S.A.

VIRGATICUTIS Roselt and Schneider, 1969.

Virgaticutis brevivirgata Roselt and Schneider, 1969, p. 71, 72, fig. 23 , pl. 14, fig. 2; cuticle, undetermined dicotyledon; middle Miocene; Nochten, lower Lusatia, Germany.

VIRGIMPARICUTIS Roselt and Schneider, 1969.

Virgimparicutis iugata Roselt and Schneider, 1969 , p. 76-77, fig. 26; pl. 17 , figs. 3,4 ; cuticle, probably Cruciferae; middle Miocene; Sedlitz, lower Lusatia, Germany.

VITTOPHYTON Vologdin, 1962.

Vittophyton parvum Vologdin, 1962, pt. 1, p. 294-295, fig. 76a; pl. 71, figs. $2 \mathrm{~b}, 3$; stromatolite, Plexostromataceae; right bank of the Nizhney Tunguska, about $34 \mathrm{~km}$ from the mouth, Turukhansk region, U.S.S.R.

VOLOGDINA Korde, 1973.

Vologdina verticilata Korde, 1973, p. 137-138, fig. 6 ; pl. 16, fig. 1 ; pl. 25, fig. 1 ; algae, Cambrinaceae; Lower Cambrian; Bazaikha river, eastern Sayan U.S.S.R.

VOLTZIOSTROBUS GrauvogelStamm, 1969.

Voltziostrobus schimperi GrauvogelStamm, 1969, p. 105-112, figs. 6a, b, 7a, 8a-m, 10a, b; pl. 3, figs. 1-11; coniferous male cone; Lower Triassic; Vosges, Lorraine, eastern France.

VOYRIOSEMINITES Trivedi and Chaturvedi, (1971) 1972.

Voyrioseminites magnus Trivedi and Chaturvedi (1971) 1972, p. 161-164, figs. 1, 2; pl. 1, fig. 1 ; seed, ?Gentianaceae; Eocene; about 10 miles west of Kuala Lumpur, Malaya.

\section{W}

WALCHIARAUCARIA Bock, 1969.

Walchiaraucaria permiensis Bock, 1969 , p. 302-308, figs. 518-527; coniferous foliage (?Araucariaceae) ; Permian; Placerville, Colorado, U.S.A.

WANKIEA Lacey and Huard-Moine, 1966.

Wankiea bondii Lacey and HuardMoine, 1966, p. 22, fig. 3; pl. 4, figs. 30,31 ; branching fructification; Lower Permian; Wankie beds, southern Rhodesia.

WATTIA Mamay, 1967.

Wattia texana Mamay, 1967, p. C124-C125, fig. 2f, $h-k$; axes, foliar appendages, systematic affiliation indetermined; Lower Permian; Baylor County, north-central Texas, U.S.A.

WATTIEZA Stockmans, 1968.

Wattieza givetiana Stockmans, 1968, p. 13-14, fig. 1; pl. 2, figs. 7, 7a; stem, Psilophytales; Middle Devonian; Brabant Massif region, central Belgium.

WEISSIA Rothwell and Taylor, 1971a.

Weissia kentuckiense Rothwell and Taylor, 1971a, p. 215-224, figs. 1-20; calamitean cones; upper Paleozoic; eastern Kentucky, U.S.A.

WEISSISTACHYS Rothwell and Taylor, 1971.

Weissistachys kentuckiense (Rothwell and Taylor, 1971) Rothwell and Taylor, $1971 \mathrm{~b}$, a new name for Weissia kentuckiense, p. 371372; calamitean cones; upper Paleozoic; eastern Kentucky, U.S.A.

WEYLANDICUTIS Schneider, 1969.

Weylandicutis marcodurense ( $\mathrm{Kr}$. and Wld., 1959) Schneider 1969, p. 16-17, pl. 3, figs. 1-5; cuticle, Ericaceae; middle Miocene; upper Lusatia, east-central Germany.

WILLIAMSONIANTHUS Kräusel and Schaarschmidt, 1966.

Williamsonianthus keuperianus Kräusel and Schaarschmidt, 1966, p. 19-22, pl. 5, figs. 7, 8; pl. 6; pl. 7, figs. 1-8; bennettitalean pollen organ; Middle Triassic; Switzerland.

WILLISIA Wolfe, 1968.

Willisia rentonensis Wolfe, 1968, p. 24-25, fig. 19 ; pl. 7, figs. 3-5; leaves, Tilliaceae; lower Tertiary; King County, Washington, U.S.A. 
WINDOSOPORELLA Mamet and Rudloff, 1972.

Windosoporella radiata Mamet and Rudloff, 1972 , p. 83 pl. 3, figs. 1825, calcareous algae, Dasycladaceae; Carboniferous; Antigonish Milk Plant, Nova Scotia, Canada. WINGATEA Ash, 1969.

Wingatea plumosa (Daugherty) Ash 1969, p. D38-39 fig. 17; pl. 4, fig. 1; fern; Chinle Formation, Upper Triassic; Fort Wingate area, New Mexico, U.S.A.

\section{Y}

YAKUTINA (Korde, 1957) Korde, 1973.

Yakutina aciculata (Korde, 1957) Korde, 1973, a new name for Sibiriella Korde; p. 242, fig. 58; algae, Seletonellaceae; Middle Cambrian; Amga river, $1 \mathrm{~km}$ beyond Khomustakh village, Yakutsk, U.S.S.R.

YOREKIELLA Krasilov, 1973.

Yorekiella pusiella Krasilov, 1973, p. 101 , pl. 49 , figs. $87-94$; pl. 50 , figs. 95-97; shoot with leaves, bryophyte, Musci; Early Cretaceous; Bureya basin, Bolshoi Yorek river, U.S.S.R.

YORKOXYLON Vogellehner, 1968.

Yorkoxylon cedroides (Holden, 1913) Vogellehner, 1968, p. 151152 ; gymnospermous wood, Protopinaceae; Jurassic; Yorkshire, England.

YUKONELLA Mamet and Rudloff 1972.

Yukonella bamberi Mamet and Rudloff, 1972 , p. 87, pl. 5, figs. 24-29; calcareous algae; Carboniferous; Sedgwick Mt., Yukon Territory, Canada.

\section{$\mathbf{Z}$}

ZAPORELLA Rácz, (1964) 1966.

Zaporella cantavriensis (1964) 1966 , p. 102-103, pl. 1, figs. 4-7; calcareous green algae, ?Dasycladaceae; the San Emiliano and LoisCiguera Formations, Carboniferous; Leon province, northwestern Spain. 


\section{BIBLIOGRAPHY}

Andrews, H. N., Jr., 1970, Index of generic names of fossil plants, 1820-1965: U.S. Geol. Survey Bull. 1300, 354 p.

Andrews, H. N., Read, C. B., and Mamay, S. H., 1971, A Devonian lycopod stem with well-preserved cortical tissues: Palaeontology, v. 14, pt. 1, p. 1-9, 3 figs., 4 pls.

Anisimova, O. I., 1973a, Bambakia-Novyy rod iz Srednekamennougol'nykh otlozheniy Severnogo Kavkaza [Bambakia-New genus from middle Carboniferous deposits of North Caucasus]: Geol. Zhur. v. 33, no. 6, p. 140-141, 1 fig.

-1973b, Novyy rod Caucasia iz srednego karbona Severnogo Kavkaza [New genus Caucasia from the middle Carboniferous of the North Caucasus] : Paleont. Zhur. 1973, no. 2, p. 138-139, 2 figs.

Antropov, I. A., 1967, Vodorosli Devona i Nizhnego Karbona (Turne) tsentral'noi chasti Vostoka Russkoi platformy in Iskopaemye Vodorosli SSSR [Algae of the Devonian and lower Carboniferous (Tournasian) of the central part of the east Russian platform, in Fossil algae U.S.S.R]: Akad. Nauk SSSR, Sibirskoe Otdel., Inst. Geologii i Geofiziki, p. 118-125, pls. 27, 28.

Archangelsy, Sergio, 1966, New gymnosperms from the Tico flora, Santa Cruz province, Argentina: British Mus. (Nat. History) Bull., Geology, v. 13, no. 5, p. 261-295, figs. 1-38, pls. 1-8, 1 map.

Archangelsky, Sergio, and De La Sota, E. R., 1966, Estudio anatomico de una neuva Lycopsida del Permico de Bolivia: La Plata Univ. Nacb, Mus. Revista, new ser., Sec. Paleontología. v. 5, no. 29, p. 17-26, 1 fig., 2 pls. Asama, Kazuo, 1970, Evolution and classification of Sphenophyllales in Cathaysia Land: Nat. Sci. Mus., Tokyo, Bull., v. 13, no. 2, p. 291-317, 4 figs., 7 pls.

Ash, S. R., 1969, Ferns from the Chinle Formation (Upper Triassic) in the Fort Wingate area, New Mexico: U.S. Geol. Survey Prof. Paper 613-D, p. D1-D52, figs. 1-19, pls. 1-5.

1970 , A problematical new plant genus from the Upper Triassic of the southwestern United States: Palaeontology, v. 13, pt. 4, p. 646-663, pls. 122-124.

1972a, Late Triassic plants from the Chinle Formation in northeastern Arizona: Palaeontology, v. 15, pt. 4, p. 598-618, 6 figs., pls. 114-119.

1972b, Marcouia, gen. nov., a problematical plant from the Late Triassic of the southwestern U.S.A.: Palaeontology, v. 15, pt. 3, p. 423-429, fig. 1A-E; pl. 80, figs. 1-9.

Awasthi, N., 1966, Fossil woods of Anacardiaceae from the Tertiary of south India: Palaeobotanist (Lucknow, India), v. 14, no. 1-3 (1965) p. 131143 , figs. $1-15$; pls. $1-4$.

1969, A new fossil wood belonging to the family Alangiacea from the Tertiary of south India: Palaeobotanist (Lucknow India), v. 17, no. 3 (1968), p. 322-325, 1 pl., 6 figs.

1970 , On the occurrence of two new fossil woods belonging to the family Lecythidaceae in the Tertiary rocks of south India: Paleobotanist (Lucknow, 
India), v. 18, no. 1 , (1965) p. $67-74$, figs. $1-10$; pls. $1,2$.

Banks, H. P., Bonamo, P. M., and Grierson, J. D. 1972, Leclercqia complexa gen. et sp. nov., a new lycopod from the late Middle Devonian of eastern New York: Rev. Palaeobotany and Palynology, v. 14, no. 1-2 (Spec. issue: Advances in Paleozoic Botany), p. 154-169, 2 figs.; 4 pls.

Banks, H. P., and Davis, M. R., 1969, Crenaticaulis, a new genus of Devonian plants allied to Zosterophyllum, and its bearing on the classification of early land plants: Am. Jour. Botany, v. 56, no. 4, p. 436-449, 35 figs.

Barale, Georges, 1972, Rhaphidopteris nouveau nom de genre de feuillage filicoïde mésozoïque: Acad. Sci. [Paris] Comptes rendus, Ser. D, Sci. Nat., v. 274, no. 7 , p. 1011-1014.

Barnard, P. D. W., and Long, A. G., 1973, On the structure of a petrified stem and some associated seeds from the Lower Carboniferous rocks of East Lothian, Scotland: Royal Soc. Edinburgh Trans., v. 69, no. 4, p. 91-108, 5 figs., 4 pls.

Barthel, Manfred, 1968, "Pecopteris" feminaeformis (Schlotheim) Sterzel und "Araucarites" spiciformis Andrae in Germar-Coenopterideen des Stephans und Unteren Perms: Paläontologische Abh., Abt. B-Paläobotanik, v. 2, no. 4, p. 727-733, 4 figs., 4 pls.

Baxter, R. W., 1967, Verticillaphyton paradoxum, a problematical fossil genus of Pennsylvanian age from Iowa coal balls: Kansas Acad. Sci. Trans., v. 70 , no. 1 , p. $71-76,17$ figs.

1971, Carinostrobus foresmani, a new lycopod cone genus from the Middle Pennsylvanian of Kansas: Palaeontographica, Abt. B, v. 134, pt. 4-6, p. 124-130, 2 figs., 2 pls.

Beck, C. B. 1967, Eddya sullivanensis, gen. et sp. nov., a plant of gymnospermic morphology from the Upper Devonian of New York: Palaeontographica, Abt. B, v. 121, pt. 1-3, p. 1-22, 25 figs., 7 pls.

Beck, C. B., and Bailey, R. E., 1967, Plants of the New Albany shale. IIIChapelia campbellii gen. n.: Am. Jour. Botany, v 54. no. 8, p. 998-1007. 27 figs.

Bertrand-Sarfati, Janine, 1972, Paleoecologie de certains stromatolites en recifs des formations du Precambrien superieur du groupe d'Atar (Mauritanie, Sahara occidental) ; creation d'especies nouvelles: Palaeogeography, Palaeoclimatology, Palaeoecology, v. 11, no. 1, p. 33-63, 17 figs.

Bilgütay, Utarit, 1968, Some Triassic calcareous algae from Plackles (Hohe Wand, Lower Austria): Geol. Bundesanstalt Verh. (Vienna), Jahrg. 1968, no. 1-2, p. 65-79, 6 figs., 3 pls.

Bock, Wilhelm, 1969, The American Triassic flora and global distribution: Geol. Center Research Ser., v. 3 and 4, 406 p., 639 figs.

Boersma, Miente, 1969, A fertile leaf of "Mariopteris latifolia": [Netherlands] Geol. Rijks Dienst. Med., new ser., no. 20, p. 65-70, 2 figs., 4 pls.

1972, The heterogeneity of the form genus Mariopteris Zeiller; a comparative morphological study with special reference to the frond composition of West-European species: Netherlands, Utrecht State Univ., Laboratory Palaeobotany and Palynology, doctoral thesis. Text and atlas, 172 p., 21 figs., 43 pls.

Boersma, Miente, and Visscher. H., 1969. On two Late Permian plants from southern France: [Netherlands] Rijks Geol. Dienst. Med., new ser., no. 20, p. 57-59, 3 figs., 2 pls.

Bohlin, Birger, 1971, Late Palaeozoic, plants from Yüerhhung, Kansu, China: Sci. Exped. Northwestern Provinces China, Repts., IV, Palaeobotany 1 
(Sino-Swedish Exped., Pub. 51), pt. 1 (text) 150 p.; pt. 2 (plates), 25 photo pls., 27 drawing pls.

Bose, M. N., and Srivastava, S. C., 1970, Glottolepis rugosa gen. et sp. nov. from Triassic beds of Nidpur: Palaeobotanist (Lucknow, India), v. 18, no. 2, (1969) p. 215-217, figs. 1a-c; pl. 1, figs. 1-9.

$1973 \mathrm{a}$, Nidistrobus gen. nov., A pollen-bearing fructification from the Lower Triassic of Gopad river valley, Nidpur: Geophytology (Lucknow, India), v. 2, no. 2, (1972) p. 211-212, 1 pl.

1973b, Some micro- and megastrobili from the Lower Triassic Gopad river valley, Nidpur: Geophytology (Lucknow, India), v. 3, no. 1, p. 69-80, 8 figs. 3 pls.

Bronn, H.G., 1858, Beiträge zur Triasischen Fauna und Flora der bituminösen Schiefer, von Raibl. III. Zur Flora der bituminösen Schiefer: Neues Jahrb. für Mineralogie, p. 1-32, 129-144, pls. 1-10.

Burakova, A. T., 1971, Sogdiania abdita iz srednevurskikh otlozheniy Sredney Azii voznozhnyy predok tsvetkovykh rasteniy [Sogdiania abdita from the Middle Jurassic formations of Central Asia-a possible ancestor of the flowering plants]: Voprosy Paleontologii, v. 6, p. 3-7, $1 \mathrm{pl}$.

Bůžek, Cestmir, 1971, Tertiary flora from the northern part of the Pětipsy area (north Bohemian basin) : Czechoslovakia, Ustredni ústav Geologický, Rozpravy, 118 p., 17 figs., 52 pls.

Chandler, M. E. J., 1966, Fruiting organs from the Morrison Formation of Utah, U.S.A.: British Mus. (Nat. History) Bull., Geology, v. 12, no. 4, p. 137-171, 46 figs., 12 pls., 157 figs.

Chirkova-Zalesskaya, E. F., 1959, Delenia terrigennogo Ural-Povolzh'ya na osnovanii iskopaemykh rastenie [Subdivision of terrigenous Devonian of the Ural-Volga land, based on fossil plants]: Moscow, Akad. Nauk SSSR, Inst. Nefti, 136 p., 150 figs., 41 pls.

Chitaley, S. D., and Kate, U. R., 1972, A petrified wood from the Deccan Intertrappean beds of Mohgaonkalan: Botanique (Nagpur, India), v. 3, no. 1 , p. $41-44,1$ pl., 6 figs.

Chitaley, S. D., and Namburdi, E. M. V., 1973, Harrisocarpon sahnii gen. et sp. nov. from the Deccan Intertrappean beds of Mohgaon-kalan, district Chhindwara: Geophytology (Lucknow, India), v. 3, no. 1, p. 36-41, 4 figs., 1 pl., 6 figs.

Chitaley, S. D., and Sheikh, M. T., 1972, Surangea mohgaoense gen. et sp. nov., a pteriodophytic fructification from Deccan Intertrappean beds of India: Geophytology (Lucknow, India), v. 1, no. 2, (1971) p. 123-126, figs. 1-4, pl. 1 .

1973, Harrisostrobus intertrappea gen. et sp. nov. A petrified gymnospermous cone from the Deccan Intertrappean beds of India: Palaeontographica. Abt B, v. 144, pt. 1-2, p. 25-30, 7 figs. pls. 10, 11.

Christophel, D. C., 1973, Sciadopityophyllum canadense gen. et sp. nov.-A new conifer from western Alberta: Am. Jour. Botany, v. 60, no. 1, p. $61-66,10$ figs.

Cloud, P. E., Jr., and Semikhatov, M. A., 1969, Proterozoic stromatolite zonation: Am. Jour. Sci., v. 267, no. 9, p. 1039-1040, fig. 8, pl. 4, figs. 1-3.

Conert, H. J., and Schaarschmidt, Friedmann, 1970, Zur Nomenklatur der Palaozoischen Gattung Tristachya Lilpop 1937 (Sphenopsida): Taxon, (Internat. Assoc. Plant Taxonomy Journ.) v. 19, no. 5, p. 793-795. 
Conrad, M. A., 1970, Barremian and lower Aptian Dasycladaceae in the area surrounding Geneva: Geologico Romana, v. 9, p. 66-100, 12 figs., 11 pls.

Conti, Sergio, 1947, Contributo allo studio delle Corallinaceae del Terzario Italiano. 2, Corallinacee del Miocene Ligure-Piemontese: Palaeontographica Italica, v. 41-42 (new ser. v. 11), anno 142-146, p. 37-61, figs. 1-3, pls. 5-8 (1-4).

De Castro, Piero, 1969, Su alcune tallofite del Mesozoico in Campania: Soc. Natur. Napoli Boll., v. 78, pt. 1, p. 87-167, 10 figs., 20 pls.

Delevoryas, Theodore, and Gould, R. E., 1971, An unusual fossil fructification from the Jurassic of Oaxaca, Mexico: Am. Jour. Botany, v. 58, no. 7, p. 616-620, 8 figs.

Delevoryas, Theodore, and Hope, R. C., 1971, A new Triassic cycad and its phyletic implications: Postilla, no. 150, p. 1-20, 12 figs.

- 1973, Fertile coniferophyte remains from the Late Triassic Deep River basin, North Carolina: Am. Jour. Botany, v. 60, no. 8, p. 810818, 28 figs.

Dorofeev, P. I., 1970, Tretchnye flory Urals [Tertiary flora of the Urals]:

Leningrad, Akad. Nauk SSSR, Botanicheskii Inst., 64 p., 14 figs., 16 pls.
$1973, \mathrm{~K}$ sistematike predkovykh form Brasenia [Systematics of the ancestral forms of Brasenia]: Paleont. Zhur., 1973, no. 2, p. 103-111, 2 figs., pl. 12 (opposite p. 101), figs. 1-15.

Douglas, J. C., 1969, The Mesozoic floras of Victoria, pts. 1 and 2: Victoria Geol. Survey Mem. 28, 310 p., 8 maps, 6 figs., 51 pls.

Dragastan, Ovidiu, 1967, Alge calcaroase in Jurasicul Superior si Cretacicul Inferior din Muntii Apuseni: Acad. Republicii Populare Romine, Studii si cercetāri, geologie, geofizicā, geografie Ser. Geologie, v. 12, no. 2, p. $441-454,8$ pls.

1969, Algues calcaires du Jurassique Supérieur et du Crétacé Inférieur de Roumanie: Rev. Micropaléontologie, v. 12, no. 1, p. 53-62, 3 pls.

1971, New algae in the Upper Jurassic and Lower Cretaceous in the Bizaz Valley, East Carpathians (Rumania) : Rev. Espanola Micropalaeontologia, v. 3, no. 2, p. 155-192, 3 figs., 12 pls.

1973, Un nouveau genre dans le Crétacé inférieur du bassin septentrional de Babadag-Dobrogea (Roumanie): Soc. Physique et d'Histoire Nat. Genève, Compte Rendu Seances, new ser., v. 7, nos. 2-3 (Suppl. to Archives des Sciences, Geneva v. 25, 1972), p. 82-87, 3 figs.

Edwards, Dianne, 1968, A new plant from the lower old Red Sandstone of South Wales: Palaeontology, v. 11, pt. 5, p. 683-690, pls. 130-132.

- 1970, Fertile Rhyniophytina from the Lower Devonian of Britain: Palaeontology, v. 13, pt. 3, p. 451-461, pls. 84, 85.

Eggert, D. A., and Delevoryas, Theodore, 1967. Studies of Paleozoic ferns: Sermaya gen. nov. and its bearing on filicalean evolution in the Paleozoic: Palaeontographica, Abt. B, v. 120, pt. 5-6, p. 169-180, pl. 34-38.

Eggert, D. A., and Taylor, T. N., 1966, Studies of Paleozoic ferns-On the genus Tedelea, gen. nov.: Palaeontographica, Abt. B, v. 118, pt. 1-3; p. 52-73, 8 figs., pls. 8-11.

- 1971, Telangiopsis gen. nov., an Upper Mississippian pollen organ from Arkansas: Bot. Gaz., v. 132, no. 1, p. 30-37, figs. 1-8.

Elias, M. E., 1942, Tertiary prairie grasses and other herbs from the high plains: Geol. Soc. America Spec. Paper 41, 176 p., 17 pls.

Elias, M. K., 1966, Living and fossil algae and fungi, formerly known as 
structural parts of marine bryozoans: Palaeobotanist, (Lucknow, India), v. 14, no. 1-3 (1965) p. 5-18, pls. 1, 2.

Elliott, G. F., 1968, Three new Tethyan Dasycladaceae (calcareous algae): Palaeontology, v. 11, pt. 4 , p. 491-497, pls. 93-95.

1970a, Calcareous algae new to the British Carboniferous: Palaeontology, v. 13 , pt. 3 , p. $443-450$, pls. $81-83$.

1970b, New and little-known Permian and Cretaceous Codiaceae (Calcareous algae) from the Middle East: Palaeontology, v. 13, pt. 2, p. 327333 , pls. 61,62 .

1970c, Pseudaethesolothon, a calcareous alga from the Fars (Persian Miocene) : Geol. Romana, v. 9, p. 31-46, 2 figs., 4 pls.

1970d, Pseudocymopolia, a Mesozoic Tethyan alga (family Dasycladaceae) : Palaeontology, v. 13, pt. 2, p. 323-326, pl. 60.

1971, A new fossil alga from the English Silurian: Palaeontology, v. 14, pt. 4, p. 637-641, 6 figs., 2 pls.

1972a, Cretacicrusta gen. nov., a possible alga from the English Cretaceous: Palaeontology, v. 15, pt. 3, p. 501-503, pls. $100,101$.

$1972 b$, Lower Paleozoic green algae from southern Scotland, and their evolutionary significance: British Mus. (Nat. History) Bull., Geology, v. 22 , no. 4 , p. $355-376,1$ fig., 10 pls.

Endlicher, Stephano, 1847, Synopsis Coniferarum: Sangalli, 368 p.

Endô, Riuji, 1968, Fossil algae from Mindoro Oriental Province, Mindoro Island, the Philippines, in Kobayashi, Teiichi, and Toriyama, Ryuzo, eds., Geology and palaeontology of Southeast Asia, V. 4: Tokyo, Univ. Tokyo Press, p. 211-219, 2 pls.

- 1969, Fossil algae from the Khao Phlong Phrab district in Thailand in Kobayashi, Teiichi, and Toriyama, Ryuzo, eds., Geology and palaeontology of Southeast Asia, V. 7: Tokyo, Univ. Tokyo Press, p. 33-85, 4 figs., pls. 5-42.

Fairon, Muriel, 1967, L'Asterovylon Elberfeldense Krausel et Weyland porte-til axes terminaux du type Hostimella Hostimensis Potonié et Bernard?: Soc. Géol. Belgique Ann., Mém. in-4 $4^{\circ}$, v. 10 (1966-67) 30 p., 10 figs., 4 pls.

Fairon, Muriel, 1967, L'Asteroxylon Elberfeldense Krausel et Weyland porte-tform genus from the Middle Devonian of Belgium: Acad. royale Belgique (Classe Sci.), 5th ser., v. 55, no. 4, p. 372-396, 18 figs.

Fedotov, V. V., 1970, Novye vidy aspidievykh i polipodivykh paporotnikov $\mathbf{s}$ sokhranivshimisya sporonsheniyami iz Paleogena Zee-Bureinskoy Depresii [New species of aspidaceous and polypodiaceous ferns with sporangia from the Paleogene of the Zeya-Bureya depression]: Paleont. Zhur., 1970, no. 4 , p. $92-98$, pl. 15 .

Fischer, Jean-Claude, and Thierry, Jacques, 1971, Revision de quelques Dasycladacees jurassiques et proposition d'un genre-Coniporella: Mus. Nat. Historic Nat. Bull., 3d ser., no. 19, Sci. Terre 3, p. 25-34, 7 figs.

Genkina, R. Z., 1966, Iskopaemaya flora i stratigrafiya nizhnemezozoiskikh otlozhenii Issyk-Kul'skoi vpadiny [Fossil flora of the Lower Mesozoic deposits of Issyk-Kul depression]: Moscow, Akad. Nauk SSSR, Institut Geologii i Razrabotki Goryuchikh Iskopaemykh, 148 p., 61 pls.

Gensel, P. G., 1973, A new plant from the Lower Mississippian of southwestern Virginia: Palaeontographica, Abt. B, v. 142, pt. 4-6, p. 137-153, 6 figs. pls. 33-40.

Gensel, P. G., Kasper, Andrew, and Andrews, H. N., 1969, Kaulangiophyton, a new genus of plants from the Devonian of Maine: Torrey Bot. Club Bull., v. 96, no. 3, p. 265-276, figs. 1-8. 
Gevin, Pierre, Koeniguer, Jean-Claude, and Lemoigne, Yves, 1973, Les bois fossiles du Dalaat el Admia (région de Tindouf, Algérie): Soc. Géol. France Bull., 7th ser., v. 13, no. 3/4, p. 386-393, 4 figs., pls. 23-25.

Giessen, M. S., 1971, Die Eözane Flora von Messel bei Darmstadt, I. Lauraceae: Palaeontographica, Abt. B, v. 134, pt. 1-3, p. 1-60, 5 figs, 10 fold-in pls., 15 pls.

Givlescu, Răzvan, 1970, Observations nouvelles sur Byttneriophyllum tiliaefolium (Al Braun) Knobloch et Kvaček: Rev. Palaeobotany and Palynology, v. 10 , no. 3 , p. 233-242, 3 pls.

Gnilovskaya, M. B., 1971, Drevneyshiye vodnyye rasteniya venda Russkoy platformy (pozdniy Dokembriy) [The oldest aquatic plants of the Wendian of the Russian Platform (late Precambrian)]: Paleont. Zhur., 1971, no. 3, p. 101-107, pl. 11, 8 figs.

- 1972, Izvestkovye vodorosli srednego i pozdnego ordovika vostochnogo Kazakhstana [The calcareous algae of the Middle and Late Ordovician of eastern Kazakhstan]: Leningrad, Akad. Nauk SSSR, Inst. Geologii i Geokhronologii Dokembriya, 196 p., 58 figs., 15 pls.

Gottwald, Helmut, 1966, Eözane Hölzer aus der Braunkohle von Helmstedt: Palaeontographica, Abt. B, v. 119, pt. 1-3, p. 76-93, 1 fig., pls. 22-24.

1969, Zwie Kisselhölzer aus den Oligozan von Tunis, Bombacoxylon oweni und Pseudolachnostyloxylon weylandii: Palaeontographica, Abt. B, v. 125, pt. 4-6, p. 112-118, pl. 23, 12 figs.

Gould, R. E., 1970, Palaeosmunda, a new genus of siphonostelic osmundaceous trunks from the Upper Permian of Queensland: Palaeontology, v. 13, pt. 1 , p. $1-28,8$ pls.

Grambast, Louis, 1966, Structure de l'utricule et phylogénie chez les Clavatoracés: Acad. Sci., Paris, Comptes Rendus, ser. D, v. 262, p. 2207-2210, 3 pls.

_ 1969, La symétrie de l'utricule chez les Clavatoracees et sa signification phylogenetique: Acad. Sci., Paris, Comptes Rendus, ser. D, v. 269, no. 9 , p. $879-881,4$ pls.

Grambast, Louis, and Lorch, Jacob, 1968, Une flore de Charophytes du Crétacé Inférieur du Proche-Orient: Naturalia monspeliensia, sér. Bot., no. 19 , p. $47-56$, pls. $1-4$.

Grambast-Fessard, Nicole, 1966, Paléobotanique, pt. 4. Contribution a l'étude des flores Tertiares des régions Provençales et Alpines-Deux bois de Dicotylédones du Pontien de Castellane: Soc. Géol. France Mém. 105, p. 131-145, 6 figs., 4 pls.

Grauvogel-Stamm, Léa, 1969, Nouveaux types d'organes reproducteurs males de Conifères du Gres à Voltzia (Trias Inférieur) des Vosges: AlsaceLorraine Serv. carte géol. Bull., v. 22, no. 2, p. 93-120, 13 figs. 3 pls.

Greguss, Pál, 1967, Fossil gymnosperm woods in Hungary from the Permian to the Pliocene: Budapest, Akad. Kaidó, 136 p., 86 pls., 14 maps in text.

1969, Tertiary angiosperm woods in Hungary: Budapest, Akad. Kaido, 151 p., 18 maps in text, 723 microphotos on 93 pls.

- 1970, Ein Callitris-ahniliches Holz aus dem Tertiar von Limburg (Niederlande): Senckenbergiana Lethaea, v. 51: nos. 2/3, p. 265-272, 2 pls.

- 1973, Die Neue Systematische Stellung Von Cycadinioxylon Czeczotti Zalewska/Miözan, Turów, Vr Polen, Und Vergleidhende Untersuchungen Mit Pseudotaxodioxylon Jaehnicheni Greguss N.G, N. Sp./Miözan, Niederlausitz, DDR.: Palaeontographica, Abt. B, v. 143, pt. 1-4, p. 1-17, 11 pls. 
Gupta, K. M., 1971, Investigations on the Jurassic flora of Rajmahal Hills, India-9. On the structure and affinities of Sewardioxylon sahnii gen. et sp. nov. Gupta, a petrified cycadean wood from the Rajmahal Hills, India: Palaeontographica, Abt. B, v. 131, pt. 5-6, p. 160-166, figs. 1, 2; pls. 35, 36, figs. 1-19.

Hall, J. W., 1969, Studies on fossil Azolla: Primitive types of megaspores and massulae from the Cretaceous: Am. Jour. Botany, v. 56, no. 10, p. 1173-1180, 30 figs.

Herbst, Rafael, 1966, Nuevos elementos de la flora fósil de la Formación Banqueró, Santa Cruz, Patagonia: Tucuman. Univ. nac., Acta Geol. Lilloana, v. 8, p. 75-86, 7 figs., 3 pls.

Huard, Jean, 1966, Paléobotanique, pt. 1. Étude anatomique des bois de Conifères des couches à lignite néogènes des Landes: Soc. Géol. France Mém. 105, v. 45, p. 5-85, 25 figs., 8 pls.

Hueber, F. M., 1970, Rebuchia: A new name for Bucheria Dorf: Taxon (Internat. Assoc. Plant Taxonomy, Jour.), v. 19, no. 5, p. 822.

1971a, Astralocaulis-A new name for Schizopodium Harris: Taxon, v. 20 , no. 4 , p. $640-641$.

1971b, Sawdonia ornata-A new name for Psilophyton princeps var. ornatum: Taxon, v. 20 , no. 4, p. 641-642.

Ischenko, T. A., 1965, Devonskaya flora Bol'shogo Donbassa [Devonian flora of the Bol'shoy Donets basin]: Kiev, Akad. Nauk UKSSR, Inst. Geol. Nauk Trudy [a monograph] 118 p., 30 pls.

- 1968, Flora verkhov nizhengo nizov srednego devona Podol'skogo Pridenstrov'ya, in Krandievskiy, B. S., Ischenko, T. A., and Kir'yanov, V. V., Paleontologiya i stratigrafiya Nizhnego Paleozoya Volyno-Podolij [Flora from the top of the Lower-base of the Middle Devonian of Podolia Cisdnestra, in Krandievskiy, B. S., Ishchenko, T. A., Kir'yanov, Paleontology and stratigraphy of the lower Paleozoic of Volyn-Podolia]: Kiev, Akad. Nauk UKS.SR, Inst. Geol. Nauk, 124 p., 16 figs., 22 pls.

Jähnichen, Helmut, and Kahlert, Eberhard, 1972, Über eine mesozoische Flora aus der Monglischen Volksrepublik: Geologie, Jahrg. 21, no. 8, p. 9641001, 5 figs., $6 \mathrm{pl}$.

Jain, R. K., and Delevoryas, Theodore, 1967, A Middle Triassic flora from the Cacheuta Formation, Minas de Petroleo, Argentina: Palaeontology, v. 10 , pt. 4 , p. 564-598, pls. 86-97.

Jennings, J. R., 1972, A new lycopod genus from the Salem Limestone (Mississippian) of Illinois: Palaeontographica, Abt. B, v. 137, pt. 1-3, p. 7284,3 figs., pls. 17-21, figs. 1-45.

Johnson, J. H., 1966, New Mississippian algae from Alberta: Jour. Paleontology, v. 40, no. 6, p. 1385-1387, pl. 176, figs. 1-10.

Kaever, Matthias, 1969, Neue Dasycladaceen-Afghanopolia fragilis n. gen., n. sp. und Cymopolia (Polytripa) paktia n. sp.- aus dem Mittel-Eozän von Ost-Afghanistan: Argumenta Paleobotanica, no. 3, 1969, p. 15-42, 8 figs., pls. 9, 10 .

Karandievskiy, V. S., Ishchenko, T. A., and Kir'yanov, V. V., 1968, Paleontologiya $\mathrm{i}$ stratigrafiya nizhnegopaleozoiya Volyno-Podolii [Paleontology and stratigraphy of the lower Paleozoic of Volyn-Podolia]: Kiev, Akad. Nauk Ukrainskoy SSR, Inst. Geol. Nauk, 124 p., 16 figs., 22 pls.

Karczewska, Jadwiga, and Ziembinska-Tworzydło, Maria, 1972, Lower Tertiary Charophyta from the Nemegt Basin, Gobi Desert: Polska Akad. Nauk, 
Palaeontologia Polonica, no. 27-Results of the Polish Mongolian Palaeont. Exped., Pt. 4, p. 51-81, pls. 7-27.

Kasper, A. E., Jr., and Andrews, H. N., Jr., 1972, Pertica, a new genus of Devonian plants from northern Maine: Am. Jour. Botany, v. 59, no. 9, p. 897-911, 18 figs.

K'hakhlov, V. A., 1964, Verkhnepalezoyskaya flora severa Sibiri. Vyp. 1, Khvoshchevye. [Upper Paleozoic flora of northern Siberia No. 1, Horsetails]: Tomsk, Tomsk Univ. Press, 112 p., 9 figs., 68 pls., 283 figs.

Khudayberdyev, R., Gomolitskii, N. P., and Lobanova, A. V., 1971, Materialy $\mathbf{k}$ yurskoy flore Yuzhnoy Fergany, in Paleobotanika Uzbekistana, v. II [Records of Jurassic flora of southern Fergana, in Paleobotany of Uzbekistan, v. II] : Tashkent, Akad. Nauk Uzbekskoy SSR, Inst. Bot., p. 3-61, pls. 1-28, 74 .

Kilpper, Karl, 1968, Koniferenzapfen aus den Tertiären Deckenschichten des Niederrheinischen Hauptflözes: Palaeontographica, Abt. B, v. 121, pts. 4-6, p. 159-168, pl. 44, 15 figs.

Kirichkova, A. I., and Budantsev, 1967, Novaya nakhodka nizhnemelovoy flory pokrytosemennymi $v$ Yakutii [A new find of Early Cretaceous flora, including angiosperms, in Yakutia]: Akad. Nauk SSSR, Vses. Bot. Obshchestvo, Bot. Zhur., v. 52, no. 7, p. 937-943, 3 figs., 2 pls.

Knobloch, Ervin, 1972, Aachenia debeyi n. g. n. sp.-eine neue Konifere aus dem Senon von Aachen: Neues Jahrbuch Geologie und Paläontologie, Monatshefte, no. 7, p. 400-406, figs. 1-10; 1 pl.

Koch, B. E., 1972a, Coryphoid palm fruits and seeds from the Danian of Nûgssuag, West Greenland: Grønlands Geol. Undersøgelse Bull. 99, and Medd. Grønland, v. 193, no. 4, 38 p., 10 figs., 18 pls.

1972b, Fossil picrodendroid fruit from the upper Danian of West Greenland: Grønlands Geol. Undersøgelse Bull. 98, and Medd. Grønland, v. 193 , no. 3,32 p., 11 figs, 24 pls.

Kochansky-Devidé, Vanda, 1970, Die Kalkalgen vom Velebit-Gebirge (Moskovien und Kassimovien): Jugoslavenska Akad. Zanosti i Umjetnosti, Palaeontologia Jugoslavice, no. 10, p. 5-30, 5 figs., 15 pls.

Koeniguer, J.-C., 1970, Étude paléoxylologique du Sahara Méridional: Congrès Natl. Soc. Savantes, 92d, Strasbourg et Colmar, 1967, Sec. Sci. Comptes Rendus, v. 3, p. 143-152, 3 figs.

-1971a, Sur les bois fossiles du Paleocene de Sessao (Niger) : Rev. Palaeobotany and Palynology, v. 12, no. 4, p. 303-323, 9 figs.; 2 pls.

1971b, Sur un bois fossile du Paleocene du Niger: Congres Natl. Soc. Savantes, 94th, Pau, 1969, Sec. Sci., Comptes Rendus v. 3, p. 157-173, 5 figs., 15 pls.

Kolakovskii, A. A., and Schakryl, A. K., 1968, Colchidia-Novyy rod Pinaceae iz Sarmata Abkhazii [Colchidia-New genus of Pinaceae from the Sarmatian of Abkhaz]: Paleont. Zhur., 1968, no. 4, p. 66-70, 1 fig., pl. 6.

Kolosov, P. N., 1966, Novye vidy Dokembryskikh vodorosley basseyna Reki Olekmy [New species of Precambrian algae from the Olekma river basin]: Akad. Nauk SSSR Doklady, v. 171, no. 4, p. 978-980, figs. 1a, b, c.

Kon'no, Enzo, 1968, Addition to some younger Mesozoic plants from Malaya, in Kobayashi, Teiichi, and Toriyama, Ryuzo, eds., Geology and palaeontology of Southeast Asia, V. 4; Tokyo, Univ. Tokyo Press, p. 139-155, 4 figs., pls. 24-28.

Kon'no, Enzo, Asama, Kazuo, and Rajah, S. S., 1970, The Late Permian 
Linggiu flora from the Gunong Blumut area, Johore, Malaysia: Nat. Sci Mus. (Tokyo) Bull., v. 13, no. 3, p. 491-580, 18 figs., 17 pls.

Korde, K. B., 1973, Vodorosli Kembriya [Cambrian algae] : Akad. Nauk SSSR, Paleontol. Inst., Trudy, v. 139, 349 p., 65 figs., 69 pls.

Krasilov, V. A., 1967, Rannemelovaya flora Yuzhnogo Primor'ya i ee znachenie dlya stratigrafii [Early Cretaceous flora of the southern Maritime Territory and its significance for stratigraphy]: Moscow, Akad. Nauk SSSR, Sibirskoe Otdel. Dal'nevostoehnyy Geol. Inst., 262 p., 38 figs., 93 pls.

1970, Listvennya pechenochniki iz Yury Bureinskogo basseina [Leafy liverworts from the Jurassic of the Bureya basin]: Paleont. Zhur., 1970, no. 3, p. 131-142, 3 figs., pls. 11, 12.

1972, Mezozoyskaya flora reki Burei (Ginkgoales i Czekanowskiales)

[Mesozoic flora of the Bureya river (Ginkgoales and Czekanowskiales)] :

Akad. Nauk SSSR, Dal'nevostochnyy Geol. Inst., 150 p., 13 figs., 34 pls.

Krasilov, V. A., 1973a, Cuticular structure of Cretaceous angiosperms from the far east of the U.S.S.R.: Palaeontographica, Abt. B, v. 142, pt. 4-6, p. 105-116, pls. $18-26$.

1973b, Mesozoic bryophytes from the Bureja basin, Far East of the U.S.S.R.: Palaeontographica, Abt. B, v. 143, pt. 5-6, p. 95-105, pls. 41-51. Kräusel, Richard, and Schaarschmidt, Friedman, 1966, Die Keuperflora von Neuewelt bei Basel.-IV. Pterophyllen und Taeniopteriden: Schweizerische Paläont. Gesell, Abh. (Mém. suisses Paléontologie), v. 84, p. 5-79, 15 figs., 15 pls.

Krylov, I. N., Korolyuk, L. K., Siderov, A. D., 1969, Stromatolity, in Razanov, A. Yu and others, Tomotski yarus i problemy nizhney granitsy Kembriya [Stromatolites, in Rozanov, A. Yu., and others, Tomotian Stage and the Cambrian lower boundary problem] : Akad. Nauk SSSR, Geol. Inst. Trudy, v. 206, p. 194-214, pls. 37-45.

Lacey, W. S., and Huard-Moine, Denise, 1966, Karroo floras of Rhodesia and Malawi. Pt. 2-The Glossopteris flora in the Wankie district of southern Rhodesia, in Symposium on Floristics and Stratigraphy of Gondwanaland: Palaeobot. Soc., Spec. Sess., Dec. 1964, Lucknow, India, p. 13-25, 3 figs., 4 pls.

Lakhnapal, R. N., and Prakash, U., 1970, Cenozoic plants from Congo. 1Fossil woods from the Miocene of Lake Albert: Koninkl. Mus. Voor Middle Afrika (Tervuren, Belgium) Annalen, Reeks in $-8^{\circ}$, Geol. Wetensch., no. 64,20 p., 13 pls.

Lakhnapal, R. N., and Verma, J. K., 1966, Fossil wood of Tetrameles from the Deccan Intertrappean beds of Mohgaonkalan, Madhya Pradesh: Palaeobotanist (Lucknow, India), v. 14, no. 1-3, (1965) p. 209-213, figs. 1, 2; pls. 1,2 ,

Lebedev, E. L., and Rasskazova, 1968, Novyy rod Mezozoiskikh paporotnikovLobifolia, in Rasteniya Mezozoya [New genera of the Mesozoic fernsLobifolia, in Mesozoic plants]: Akad. Nauk SSSR, Geol. Inst. Trudy, v. 191 , p. 56-69, 7 figs., 3 pls.

Leclercq, Suzanne, and Bonamo, P. M., 1973, Rellimia thompsonii, a new name for Milleria (Protopteridium) thompsonii Lang 1926 emend. Leclercq and Bonamo, 1971: Taxon, v. 22, no. 4, p. 435-437, fig. 1.

Leisman, G. A., and Peters, J. S., 1970, A new pteridosperm male fructification from the Middle Pennsylvanian of Illinois: Am. Jour. Botany, v. 57, Lele, K. M., 1969, Studies in the Glossopteris flora of India. 39. Alatocarpus 
gen. nov.; a new platyspermic seed from the Singrauli coalfield: Palaeobotanist (Lucknow, India), v. 17, no. 1 (1968), p. 52-58, figs. 1-10; pl. 1, figs. 1-15.

Lemoigne, Yves, 1968, Un nouveau genre de structure ligneuse de type gymnospermien: Embergerixylon nov. g.: Soc. Geol. Nord Ann., v. 88, pt. 4, p. $155-158$, pls. $13,14$.

Lemoine, Marie, 1966, Un nouveau genre d'algue du crétacé inférieur: Agardhiellopsis nov. gen.: Rev. Micropaléontologie, v. 8, no. 4, p. 203-210, fig. la-d; pl. 1, figs. 1-4.

-1969-70, Les algues Floridées calcaires du Crétacé du Sud de la France: Mus. Natl. Histoire Naturelle, Archives, Sér. 7, v. 10, p. 127-240, 15 pls.

Lemoine, Marie, et Emberger, Jacques, 1967, Kymalithon, nouveau genre de Melobesiee de l'Aptien supérieur et considerations sur l'âge du faciès à Mélobésiées dit "faciès de Vimport": Soc. Linéenne Bordeaux Actes, v. 104, ser. B, no. 9 , p. 3-14, 4 pls.

LeMone, D. V., and Johnson, R. E., 1969, Neogene flora from the Rincon hills, Dona Ana County, New Mexico: New Mexico Bur. Mines and Mineral Resources Circ. 104, p. 77-88, 1 fig., 4 pls.

Lepekhina, V. G., 1969, Paleoksilologicheskaya kharakteristika verkhnepaleozoiskikh uglenosnykh otlozhenii Kuznetskogo basseina [Paleoxylotomous character of the upper Paleozoic coal-bearing deposits of the Kuznets basin] Vsesoyuz. Nauchno-Issled., Geol. Inst., Biostratigraficheskii Sbornik, No. 4 p. 126-139, 6 pls.

Loubière, A. 1956, Sur un nouveau genre de Mesocaryales Paléozoiques. Mus. Natl. Histoire Nat. Bull., 2d ser., v. 28, no. 5, 492-494, 3 figs.

Louvet, Paul, 1967, Sur une Meliaceae fossile nouvelle du Tertiaire d'Algérie Tinrhert) : Congrès Natl. Soc. Savantes, 91st Rennes, 1966, Sec. Sci., Comptes rendus v. 3, p. 186-197, 2 figs., 1 pl.

MacGinitie, H. D., 1969, The Eocene Green River flora of northwestern Colorado and northeastern Utah: California Univ. Pubs. Geol. Sci., v. 83, 203 p., 31 pls.

Maheshwari, H. K., 1967, Studies in the Glossopteris flora of India-[pt.] 28. On some fossil wood of the Raniganj coalfield, Bengal: Palaeobotanist (Lucknow, India), v. 15, no. 3(1966) p. 243-257, 10 figs., 4 pls.

1972, Lelstotheca: a new name for Stellotheca Surange and Prakash: Geophytology (Lucknow, India), v. 2, no. 1, p. 106.

Malich, N. S., 1966, Vysokoorganizobannye rasteniya iz Kembriyskikh otlozheniy zapadnoy chasti Sibirskoy platformy [Highly organized plants from the Cambrian deposits of the western part of the Siberian platform]: Akad. Nauk SSSR, Doklady, v. 166, no. 5, p. 1207-1209, fig. 1a-z (opposite p. 1171).

Mamay, S. H., 1966, Tinsleya, a new genus of seed-bearing callipterid plants from the Permian of north-central Texas: U.S. Geol. Survey Prof. Paper 523-E, p. E1-E15, 2 figs., 3 pls.

- 1967, Lower Permian plants from the Arroyo Formation in Baylor County, north-central Texas: U.S. Geol. Survey Prof. Paper 575-C, p. C120-C126, 2 figs.

1968, Russellites, new genus, a problematical plant from the Lower

Permian of Texas: U.S. Geol. Survey Prof. Paper 593-I, p. I1-I15, 3 pls.

1973, Archaeocycas and Phasmatocycas-new genera of Permian cycads:

U.S. Geol. Survey, Jour. Research, v. 1, no. 6, p. 687-689, fig. 1a-g.

Mamet, B. L., and Rudloff, B., 1972, Algues Carboniféres de la partie septen- 
trionale de l'Amérique du Nord: Rev. Micropaléontologie, v. 15, no. 2, p. $75-114,10$ pls.

Marguerier, Janine, 1973, Paléoxylologie du Gondwana Africain-Étude et affinities du genre Australoxylon: Palaeontologia afr., v. 16, p. 37-58, 4 figs., 6 pls.

Maslov, V. P., 1966, Nekotorye kaynozoyiskiye kharofity yuga SSSR i metodika ikh izucheniya, in Maslov, V. P. and Vakhrameev, V. A., eds., Iskopaemye kharofity SSSR [Certain Cenozoic charophytes in the south of the U.S.S.R. and the methods of their studies, in Maslov, V. P. and Vakhrameev, V. H., eds., Fossil charophytes of the U.S.S.R.] : Akad. Nauk SSSR, Geol. Inst., Trudy [new ser.], no. 43 , p. 10-89, 30 figs., 11 pls.

Masse, J.-P., Conrad, M. A., and Radoičić, Rajka, 1973, Angioporella fouryae, n. gen., n. sp., une algue calcaire (Dasycladaceae) du Barrémien du sud de la France: Eclogae Geol. Helvetiae, v. 66, no. 2, p. 383-387, 1 fig., 1 pl.

Matten, L. C., 1968, Actinuxylon banksii gen. et. sp. nov.-a progymnosperm from the Middle Devonian of New York: Am. Jour. Botany, v. 55, no. 7, p. 773-782, 19 figs.

1973, The Cairo flora (Givetian) from eastern New York. 1. Reimannia terete axes and Cairoa lamanekii gen. et sp. nov: Am. Jour. Botany, v. 60, no. 7, p. 619-630, 35 figs.

Matten, L. C., and Banks, H. P., 1966, Triloboxylon ashlandicum gen. et sp. n. from the Upper Devonian of New York: Am. Jour. Botany, v. 53, no. 10, p. 1020-1028, 18 figs.

Menendez, C. A., 1965, Sueria rectinervis n. gen. et sp. de la flora fósil de Ticó, Provincia de Santa Cruz: Ameghiniana (Asoc. Paleontológia Argentina, Rev.), v. 4, no. 3, p. 75-79, 4 pls.

Meyen, S. V., 1969a, Novye rody Entsovia i Slikovia iz Permskikh otlozhenii Russkoy Platformy i Priural'ya [New genera Entsovia and Slikovia, from Permian deposits of the Russian Platform and Cisuralia]: Paleont. Zhur., 1969, no. 4, p. 93-100, 6 figs., pls. 14, 15.

1969 b, O rode Zamiopteris Schmalhausen i ee soothoshenie s nektorymi smezhnymi rodami, in Pteridospermy Verkhnego Paleozoya i Mezozoya [On the genus Zamiopteris Schmalhausen and its relation to some adjacent genera]: Akad. Nauk SSSR, Geol. Inst. Trudy, [n.s.] v. 190, p. 85-104, 3 figs., 5 pls.

1972, Are there ligula and parichnos in Angara Carboniferous lepidophytes?: Rev. Palaeobotany and Palynology, v. 14, nos. 1/2, (Special issue: Advances in Paleozoic Botany) p. 149-157, 5 figs., pl. 1, figs. 7-10.

Miki, Shigeru, 1969, Protosequoia (n.g.) in Taxodiaceae from Pinus Trifolia beds in central Honshu, Japan: Japan Acad. Proc., v. 45, no. 8, p. 727-732, 3 figs.

Millan, J. H., 1967, Novas frutificações na flora Glossopteris do Gonduana Inferior do Brasil. Dolianitia, gen. nov: Brazil Div. Geologia e. Mineralogia, Notas Prelim. e Estudos, no. 140, 19 p., 4 pls.

Millay, M. A., and Eggert, D. A., 1970, Idanothekion gen. n., a synangiate pollen organ with saccate pollen from the Middle Pennsylvanian of Illinois: Am. Jour. Botany, v. 57, no. 1, p. 50-61, 24 figs.

Miller, C. N., and Brown, J. T., 1973, A new voltzialean cone bearing seeds with embryos from the Permian of Texas: Am. Jour. Botany, v. 60, no. 6, p. 561-569, 13 figs.

Mogucheva, N. K., and Il'ina, V. I., 1969, Novyy rod paporotnikov iz Nizhnego Triasa Tungusskoy sineklizy [A new fern genus from the Lower Triassic 
of the Tunguska syneclise]: Akad. Nauk SSSR, Sibirskoe Otdel., Geologiya Geofizika, no. 6(114), 1969, p. 131-135, 1 pl.

Morbelli, M. A., and Petriella, Bruno, 1973, "Austrostrobus ornatum" nov. gen. et sp., cono petrificado de Lycopsida del Triasico de Santa Cruz (Argentina) : Mus. La Plata Rev. (n. s.) v. 7, Paleontologia no. 46, p. 279-289, 1 fig. 2 pls.

Müller-Stoll, W. R., and Mädel, Erika, 1967, Die Fossilen Leguminosen-Hölzer eine Revision der mit Leguminosen vergleichenen fossilen Hölzer und Beschreibungen älterer und neuer Arten: Palaeontographica, Abt. B, v. 119, pt. 4-6, p. 95-174, 13 figs., pls. 25-39.

Müller-Stoll, W. R., and Mädel-Angeliewa, Erika, 1969, Actinidioxylon princeps (Ludwig) n. comb., ein Lianenholz aus de Pliozän von Dernbach im Westerwald: Senckenbergiana Lethaea, v. 50, nos. 2/3, p. 103-115, 3 figs., 2 pls.

Musacchio, E. A., 1971, Charophyta de la Formacion la Amarga (Cretaceous Inferior) Provincia de Neuquen, Argentina: Mus. La Plata Rev., new ser., v. 6, Sec. Paleontologia, no. 17, p. 19-38.

Nagy, István, 1971, A paleotrix kérdés. A fonalasalgák rétegtani szerepe a mecseki felsojúrában: Magyar Állami Földt. Int. Evi Jelentése, Az 1969, Evröl, p. 299-316, 4 pls.

Namburdiri, E. M. V., 1969, Kuprianivaites deccanii, gen. et sp. nov., A new petrified sporocarp from the Deccan Intertrappean beds of Mohgaon Kalan: Jour. Palynology (Lucknow, India), p. 51, 52, 2 figs.

Novik, E. O., 1968, Rannekamennougol'naya flora Donetskogo Basseina i ego zapadnogo prodolzheniya [Early Carboniferous flora of the Donets basin and its western extension]: Kiev, Akad. Nauk UKSSR, Inst. Geol., 234 p., 51 pls.

Obrhel, Jiri, 1968a, Maslovina meyenii, n. g. et sp.-neue Codiacea aus dem Silur Böhmens: Czechoslovakia Ústred. Ústav Geol. Vestník, v. 43, no. 5, p. 367-370, 1 fig., 2 pls.

1968b, Neue Pflanzenfunfe im mittelböhmischen Ordovizium: Czechoslovakia Ústred. Ústav. Geol. Vestník, v. 43, no. 6, p. 463-464, 1 pl.

- 1969, Die mitteldevonische Landflora von Chabičov in Mähren: Čas. Mineralogii a Geologii, v. 14, no. 1, p. 53-59, 4 pls.

Page, V. M., 1970, Angiosperm wood from the Upper Cretaceous of central California. III: Am. Jour. Botany, v. 57, no. 9, p. 1139-1144, 17 figs.

1973, A new conifer from the Upper Cretaceous of central California. Am. Jour. Botany, v. 60 , no. 6, p. 570-575, 15 figs.

Pantíc, Smiljka, 1971, Baccanella floriformis n. gen., n. sp, iz srednjeg trijasa Dinarida [Baccanella floriformis, $n$. gen. n. sp. from the Middle Jurassic of the Dinarides]: Geoloshki Anali Balkanskoga Poluostrva, v. 36, p. 105111, 2 figs., 3 pls.

Petriella, Bruno, 1969, Menucoa cazaui nov. gen. et sp., tronco petrificado de Cycadales, Provincia de Rio Negro, Argentina: Ameghiniana (Asoc., Paleontol. Argentina, Revista) v. 6, no. 4, p. 291-302, 3 figs., 1 pl.

Petryk, A. A., and Mamet, B. L., 1972, Lower Carboniferous algal microflora, southwestern Alberta: Canadian Jour. Earth Sci., v. 9, no. 7, p. 767-802, 10 figs., 10 pls.

Pettitt, J. M., and Beck, C. B., 1968, Archaeosperma arnoldii-A cupulate seed from the Upper Devonian of North America: Michigan Univ., Mus. Paleontology Contr., v. 22, no. 10, p. 139-154, 3 figs., 6 pls.

Pettitt, J. M., and Lacey, W. A., 1972, A Lower Carboniferous seed compression 
from North Wales: Rev. Paleobotany and Palynology, v. 14, nos. 1-2 (Special issue: Advances in Paleozoic Botany), p. 154-169, 2 figs., 4 pls.

Pfefferkorn, H. W., 1973, Kankakeea gen. nov., buds for vegetative reproduction in Carboniferous ferns: Paläontologische Zeitschr., v. 47, no. 3/4, p. 143-151, 2 figs., pls. 25, 26.

Pflug, H. D., 1966, Einige Reste Nieder Pflanzen aus dem Algonkium: Palaeontographica, Abt. B., v. 117, pt. 4-6, p. 59-74, 2 figs.; pls. 25-29.

Phillips, T. L., and Andrews, H. N., 1966, Catenopteris simplex gen. et sp. nov., a primitive pteridophyte from the Upper Pennsylvanian of Illinois: Torrey Bot. Club Bull., v. 93, no. 2, p. 117-128, 13 figs.

Pia, Julius, 1934, Kalkalgen aus dem Eozän der Felsen von Hričovské Podhradie im Waagtal: Czechoslovakia Státní Geol. Ústav Věstník, v. 10, no. 1-2, p. 14-18, 8 figs.

1943, Geologische Untersuchungen in der Salmgruppe (Oberdonau) : Naturhist. Mus. Wien, Annalen, v. 53, pt. 1 (1942), p. 5-155, 9 figs., pls. 1-7.

Prakash, Uttam, and Awasthi, N., 1970, Fossil woods from the Tertiary of eastern India, I: Palaeobotanist (Lucknow, India), v. 18, no. 1 (1969), p. 32-44, 1 fig., 6 pls.

Prakash, Uttam, et Boureau, Edouard, 1970, Sur une monocotylédone herbacée fossile à structure conservée des basaltes de Columbia, dans le Miocene de Vantage, central Washington, U.S.A.: Congres Natl. Soc. Savantes, 92d, Strasbourg et Colmac, 1967, Sec. Sci., Comptes rendus, v. 3, p. 93-107, 5 pls., 11 figs.

Prakash, Uttam, Březinová, Drahomíra, and Bủžek, Cestmír, 1971, Fossil woods from the Doupovské hory and Ceské stredohoi mountains, in northern Bohemia: Palaeontographica, Abt. B, v. 133, pt. 4-6, p. 103-128, pls. 30-43, 1 map.

Prakash, Uttam, and Tripathi, P. P., 1967, Fossil wood of the Lannea from the Tertiary of Assam: Current Sci. (Bangalore, India), v. 36, no. 17, p. $462-463,3$ figs.

1968, Fossil wood of Adenanthera and Swintonia from the Tertiary of Assam: Current Sci. (Bangalore, India), v. 37, no. 4, p. 115-116; 4 figs.

1970, Fossil woods from the Tertiary of Hailakandi, Assam: Palaeobotanist (Lucknow, India), v. 18, no. 1 (1969), p. 20-21, figs. 1-13, pls. 1-3.

Praturlon, A., 1966, Heteroporella lepina, new dasyclad species from Upper Cenomanian-Lower Turonian of central Äpennines: Soc. Paleontologica Italiana, Boll., v. 5, no. 2, p. 202-205, 1 fig. pls. 51, 52.

Raaben, M. E. et Zabrodin, V. E., 1972, Vodoroslevya problematica verkhnego rifeya (stromatolity, onkolity) [Algal problematica of the Upper Riphean (stromatolites, onkolites) ]: Akad. Nauk SSSR, Geol. Inst., Trudy [n.s.], no. 217, p. 1-128, 16 figs., 44 pls.

Rácz, Lászlo [1964] 1966, Carboniferous algae and their associations in the San Emiliano and Lois-Ciguera Formations (Prov. Leon, N. W. Spain): Leidse Geol. Meded., pt. 31, p. 1-112, 26 figs., 13 pls. (pre-issued Oct. 14, 1964.)

Radchenko, G. P., 1934, Materialy k podznaniyu flory Kuznetskogo Basseyna [Contribution to the knowledge of Paleozoic flora of the Kuznetzsk Basin] : Geologo-Razvedochnoe. Upravelenie Sibirskoe Otdel., Materialy, no. 13, p. 3-55, pl. 12, figs. 9-12.

Radchenko, M. I., 1967, Kamennougol'naya flora Yugo-Vostochnogo Kazakhstana 
[Carboniferous flora of southeastern Kazakhstan]: Alma-Ata, Akad. Nauk Kazakhskoy SSR, Inst. Geol. Nauk, 68 p., 25 pls.

Novyi rod Neuburgia v Rannekamennougol'nykh otlozhenyakh Kazakhstana, in Pteriodospermy Verkhnego Paleozoya i Mezozoya [A new genus Neuburgia in the Lower Carboniferous deposits of Kazakhstan, in Pteridosperms of the Upper Paleozoic and Mesozoic] Akad. Nauk SSSR, Geol. Inst. Trudy [new ser.], v. 190, p. 105-108, 2 pls.

Ramovs, Anton, 1970, Permopadina fallax nov. gen., nov. sp., eine mittelpermische Alge aus den östlichen Karawanken: Paläontologische Zeitschr., v. 44, no. 1/2, p. 97-100, 1 fig., pl. 12.

Rao, A. R., and Bose, M. N., 1971, Podostrobus gen. nov., a petrified podocarpaceous male cone from the Rajmahal Hills, India: Palaeobotanist (Lucknow, India), v. 19, no. 1, (1970) p. 83-85, pl. 1, figs. 1-14.

Rásky, Klara, 1966, Some plant remains from the Tertiary of Hungary : Palaeobotanist (Lucknow, India), v. 14, nos. 1-3 (1965), p. 264-268, pls. 1-3.

Remy, W., and Remy, R., 1969, Alstaettia andersoni n. gen., n. sp. ein Matoniaceen-Sprossrest aus dem Mittel Barrême von Alstätte/Krs. Ahaus: Argumenta Palaeobotanica, no. 3, 1969, p. 91-129, figs. 1-3, pls. 20-23.

Rigby, J. F., 1972a, The flora of the Kaloola Member of the Baralaba Coal Measures, central Queensland: Queensland Geol. Survey Pub. 352, Palaeontological Papers 26, p. 1-12, figs. A-I.

1972b, The Notocalamitaceae, a new family of upper Paleozoic equisetaleans: Palaeobotanist (Lucknow, India), v. 19, no. 2 (1970), p. 161-163, 13 text-figs., 1 pl., 5 figs.

Robison, C. R., and Person, C. P., 1973, A silicified semiaquatic dilcotyledon from the Eocene Allenby Formation of British Columbia: Canadian Jour. Botany, v. 51, no. 7, p. 1373-1378, pls. 1-4, figs. 1-20.

Roselt, Gerhard, and Schneider, Wilfrid, 1969, Cuticulae dispersae, ihre Merkmale, Nomenklatur und Klassifikation: Paläont. Abh., Abt. B-Paläobotanik, v. 3, no. 1, p. 1-128, 32 figs., 20 pls.

Rothwell, G. W., and Taylor, T. N., 1971a, Studies of Paleozoic calamitean cones-Weissia kentuckiense gen. et sp. nov.: Bot. Gaz., v. 132, no. 3, p. 215-224, figs. 1-20.

1971b, Weissistachys kentuckiensis: A new name for Weissia kentuckiense Rothwell and Taylor: Bot. Gaz., v. 132, no. 4, p. 371-372.

Samy'lina, V. A., 1967, Mezozoyskaya flora levoberezh'ya r. Kolymy (Zyryanskiy uglenosnyy basseyn), Chast II. Ginkgovye, Khvoynye. Obshchie glavy, in Takhtadzhyana, A. L., red. Paleobotanika-Problemy izucheniya iskopaemoy flory uglenosnykh otlozheniy SSSR, [Mesozoic flora of the left (west) bank of the Kolyma river (Zyrianska coal-basin), Pt. II. Ginkgoales, Coniferales. General chapters, in Takhtadzhyana, A. L., ed. Paleobotany-Problems (concerning) the studies of fossil flora in the coalbearing deposits of the U.S.S.R]: Akad. Nauk SSSR, Bot. Inst.Trudy, ser. 8, issue 6, p. 134-175, 14 pls.

1968, Early Cretaceous angiosperms of the Soviet Union based on leaf and fruit remains: Linnean Soc. London (Botany) Jour., v. 61, no. 384, p. 207-218, 3 figs.; 1 pl.

Samy'lina, V. A., and Filippova, G. G., 1970, Novye Melovye paporotniki severo-vostoka SSSR [New Cretaceous ferns from northeastern U.S.S.R.]: Paleontol. Zhur., no. 2, 1970, p. 91-97, 4 figs. pl. 12.

Saydakovskiy, L. Ya., 1968, Kharofita iz Triasa Prikaspiyskoy vpadiny 
[Charophyta from the Triassic of the Ciscaspian depression]: Paleont. Zhur., no. 2, 1968, p. 95-110, pls. 15, 16.

Schaarschmidt, Friedman, and Maubeuge, P. L., 1969, Eine mannliche Gymnospermen-Fruktifikation aus dem Voltsiensandstein (Unter Trias) der Vogesen: Senckenbergiana Lethaea, v. 50, nos. 5/6, p. 377-398, 14 figs.; 3 pls. Scheckler, S. E., and Banks, H. P., 1971, Proteokalon, a new genus of progymnosperms from the Devonian of New York State and its bearing on the phylogenetic trends in the group. Am. Jour. Botany, v. 58, no. 9, p. 874-884, 30 figs.

Schneider, Wilfrid, 1969, Cuticulae dispersae aus dem 2. Lausitzer Flöz (Miózän) und ihre fazielle Aussage: Freiberger Forschungshefte, Reihe no. 222, 75 p., 21 figs., 10 pls.

Schopf, J. M., Mencher, Ely, Boncot, A. J., and Andrews, N. H., 1966, Erect plants in the Early Silurian of Maine: U.S. Geol. Survey Prof. Paper 550-D, p. D69-D75, 2 figs.

Schultz-Motel, Jurgen, 1966, Gymnospermen-Holzer aus dem oberkretazischen Umzamba-Schichten von Ost-Pondoland (S-Afrika): Senckenbergiana Lethaea, v. 47, no. 3, p. 279-337, 2 figs., pls. 38-44.

Schumacker-Lambry, J., 1966, Étude d'un cone de Lepidocarpaceae du houiller belge: Acad. royale Belgique, Classe Sci. Mém., ser. 2, v. 17, no. 1, p. 7-27, 1 fig. 5 pls.

Schweitzer, H. J., 1968, Pflanzenreste aus dem Devon Nord-Westspitzbergens: Palaeontographica, Abt. B, v. 123, Festband Weyland, pt. 1-6, p. 43-75, pls. 5-15; 13 figs.

1969, Die Oberdevon-Flora der Bareninsel, 2. Lycopodinae: Palaeontographica, Abt. B, v. 126, pt. 4-6, p. 101-137, figs. 1-23, pls. 12-23.

1972, De Mitteldevon-Flora von Lindlar (Rheinland) 3. FilicinaeHyenia elegans Kraüsel and Weyland: Palaeontographica, Abt. B., v. 137, pt. 4-6, p. 154-175, 14 figs.. pls. 30-39.

Segonzac, Geneviève, $1970 \mathrm{a}$, Dasycladales nouvelles du Sparnacien des Pyrénées agiégeoises: Acad. Sci. (Paris), Comptes rendus, Sér. D, v. 270, no. 15, p. 1881-1884, 3 figs.

1970b, Essai de classement de quelques Acétbulariacées Tertiares (algues calcaires) : Soc. Histoire Nat. Toulouse Bull., v. 106, no. 3-4, p. 333-340, pls. $19,20$.

1972a, Description d'un nouveau genre algue calcaire du "Sparnacien" des Pyrénées, de la Haute-Garonne, de l'Aude et l'Ariège-Sarosiella: Soc. Histoire Nat. Toulouse Bull. v. 108, no. 3-4, p. 394-396, pl. 1, figs. 1-6. 1972b, Une nouvelle Acétabulariacée tertiaire-Rostroporella oviformis n. g., n. sp. (algue calcaire). Géol. Soc. France Bull., 7th Ser., v. 8, no. $1 / 2$, p. $181-186$, pls. $10,11,12$.

Senkevich, M. A., 1961, Opisanie flory Devona Kazakhstana [Description of Devonian flora of Kazakhstan]: Materialy po geologii i poleznym iskopaemym Kazakhstana, no. 1 (26), p. 115-211, 13 figs., pls. 20-37.

Sharma, B. D., 1969, Guptioxylon amarjolense gen. et sp. nov. from Amarjola in the Rajmahal Hills, India: Palaeontographica, Abt. B, v. 126, pt. 4-6, p. 145-153, 5 figs., 3 pls.

1971, On the anatomy and isolated petioles of ferns from Armajola, Rajmahal Hills, India: Linnean Soc., London, Bot. Jour., v. 64, no. 1, p. 149-156, 6 figs., 2 pls.

Shaykin, I. M., 1966, Characeae verkhnego karbona Donbassa, in Maslov, V. P., and Vakhrameev, V. A., eds., Iskopaemye kharofity SSSR [Characeae of the upper Carboniferous of the Donets basin, in Maslov, V. P., 
and Vakhrameev, V. A., eds., Fossil charophytes of the U.S.S.R.] Akad. Nauk SSSR, Geol. Inst. Trudy [new ser.], no. 143, p. 154-160, 2 figs., 1 pl. Shvedov, N. A., 1963, Novye Rannetriasovye rasteniya iz Noril'skogo raiona [New Early Triassic plants from the Noril'sk region]: Nauchno-Issled. Inst. Geol. Arktiki, Sbornik statey po paleontologii i biostratigrafii no. 32, p. 59-67, 1 fig., 3 pls.

Sikstel', T. A., 1966, K fitostratigrafii Permi Sredney Azii [The phytostratigraphy of the Permian of Central Asia]: Tashkent, Gosudarstvennyy Univ., 60 p., 16 figs., 22 pls.

Sikstel', T. A., and others, $1971, \mathrm{~K}$ istorii razvitiya ginkgovykh $\mathrm{v}$ Sredney Azii, in Paleobotanika Uzbekistana, v. II [The history of the development of ginkgophytes in Central Asia, in Paleobotany of Uzbekistan, v. II]: Tashkent, Akad. Nauk Uzbekskoy SSR, Inst. Bot., p. 62-116, 3 figs., pls. 29-71.

Skog, J. E., Andrews, H. N., and Mamay, S. H., 1969, Canipa quadrifida, a syngial fructification from the Middle Pennsylvanian of West Virginia: Torrey Bot. Club Bull., v. 96, no. 3, p. 276-287, 12 figs.

Skog, J. E., and Banks, H. P., 1973, Ibyka amphikoma, gen. et sp. n., a new protoarticulate precursor from the late Middle Devonian of New York State: Am. Jour. Botany v. 60, no. 4, p. 366-380, 24 figs.

Sokać, Branko, 1968, A new genus of calcareous algae from the Middle Triassic of Velebit: Yugoslavia, Geološki vjesnik 21 (1967), p. 207-212, 1 fig., 4 pls.

Srebrodol'skaya, I. N., 1969, Novye predstaviteli Pozdnetriasovoi flory Severnogo V'etnama, in Biostratigraficheskii Sbornik, issue 4 [New representatives of Late Triassic flora of northern Viet Nam, in Biostratigraphical articles, issue 4]: Vsesoyuz. Nauchno-Issled. Geol. Inst., Tr., new ser., v. 130a, p. 85-101, 6 figs., 5 pls.

Srivastava, S. C., and Maheshwari, H. K., 1973, Satsangia, a new plant organ from the Triassic of Nidpuri, Madhya Pradesh: Geophytology, (Lucknow, India), v. 3 , no. 2 , p. 222-227, 3 figs., 1 pl.

Stidd, B. M., and Hall, J. W., 1970, Callandrium callistophytoides, gen. et sp. nov., the probable pollen-bearing organ of the seed fern Callistophyton: Am. Jour. Botany, v. 57, no. 4, p. 394-403, 25 figs.

Stanislavsky, F. A., 1973, Novyy rod Toretzia iz verkhnego Triasa Donbassa i ego otnoshenie $\mathrm{k}$ rodami poryadka Ginkgoales [A new Upper Triassic genus Toretzia from the Donbass and its relation to the order of Ginkgoales] : Paleont. Zhur., 1973, no. 1, p. 88-96, 2 figs. pl. 12.

Stockmans, François, 1968, Végétaux Mesodevoniens recoltea aux confins su Massif du Brabant (Belgique) : Inst. Royal Sci. Naturelles Belgique, Mém. 159, 49 p., 5 figs., 14 pls.

Straus, Adolf, 1969, Beiträge zur Kenntnis der Pliozänflora von Willershausen (VII)-Die Angiospermen-Früchte und -Samen: Argumenta Palaeobotanica, Heft 3, 1969, p. 163-197, 9 figs., pls. 28-33.

Sukhov, S. V., 1969, Semena Pozdnepaleozoyskikh rasteniy Sredney Sibiri [Seed of late Paleozoic plants of central Siberia]: Sibirskii NauchnoIssled. Inst. Geologii Geofizii, i Mineralnogo Syr'ya Trudy, no. 64, 264 p., $31 \mathrm{pls}$.

Surange, K. R., and Maheshwari, H K., 1970, Some male and female fructifications of Glossopteridales from India: Palaeontographica, Abt. B, v. 129 , pt. $4-6$, p. $178-192$, pls. $40-43$, figs. 1-11.

Sveshnikova, I. N., 1967, Pozdmelovye Khvoynyye Sovetskogo Soyusa. I. Iskopaemye Khvoynyye Vilyuyskoy sineklizy, in Takhtadzhyana, A. L., red. 
Paleobotanika-Problemy izucheniya iskopaemoy flory uglenosnykh otlozheniy SSSR, [Late Cretaceous Coniferales of the Soviet Union. [pt.] IFossil Coniferales of the Viliuyian depression, in Takhtadzhyana, A. L., ed. Paleobotany-Problems (concerning) the studies of fossil flora in the coal-bearing deposits of the U.S.S.R.]: Akad. Nauk SSSR, Bot. Inst. Trudy, ser. 8 , no. 6 , p. $177-203,12$ pls.

Sveshnikova, I. N., and Budantsev, L. Yu., 1967, Nansenia-Novyy rod semeistva Pinaceae iz nizhnego mela Zemli Frantsa Iosifa [Nansenianew genus of the family Pinaceae from the Lower Cretaceous of Frans Josef Land] : Palaeontol. Zhur., 1967, no. 3, p. 123-125, pl. 12.

1969, Iskopaemye flory Arktiki, I. Paleozoyskie i Mezozoyskie flory Zapadnogo Shpitsbergena, Zemli Frantsa-Iosifa i Ostrova Novaya Sibir' [Fossil flora of the Arctic (pt.) I-Paleozoic and Mesozoic flora of west Spitsbergen, Franz-Josef Land and New Siberian Islands]: Leningrad, Akad. Nauk SSSR, Bot. Inst., 129 p., 18 figs., 46 pls.

Taylor, T. N., 1967, Paleozoic seed studies: On the structure of Conostoma leptospermum n. sp., and Albertongia incostata n. gen. and sp.: Palaeontographica, Abt. B, v. 121, pt. 1-3, p. 23-29, figs. 1-4; pls. 8, 9.

1970, Lasiostrobus gen. n., a staminate strobilus of gymnospermous affinity from the Pennsylvanian of North America: Am. Jour. Botany, v. 57 , no. 6 , pt. 1 , p. $670-690,48$ figs.

1971, Halletheca reticulatus gen. et sp. n.: A synangiate Pennsylvanian pteridosperm pollen organ: Am. Jour. Botany, v. 58, no. 4, p. 300-307, 16 figs.

Taylor, T. N., and Eggert, D. A., 1967, Petrified plants from the Upper Mississippian of North America-1. The seed Rhynchosperma gen. n.: Am. Jour. Botany, v. 54, no. 8, p. 984-992, figs. 1-25.

Tchuvashov, B. I., 1971, Novyy rod pozdnepaleozoyskikh krasnykh vodorosley [A new genus of late Paleozoic red algae]: Paleontol. Zhur., no. 2, 1971 p. $85-89$, fig. 1 ; pl. 10 , figs. $1-4$.

Teslenko, Yu. V., 1970a, $\mathrm{K}$ geologicheskoi istorii listvennits i lzhelistvennits [On the geological history of larchs and pseudolarchs]: Paleont. Zhur., no. 2, 1970, p. 98-104, pl. 13, figs. 1-6.

1970b, Stratigrafiya i flora Yurskikh otlozheniy zapadnoy i yuzhnoy Sibiri i Tuvy [Stratigraphy and flora of Jurassic deposits of western and southern Siberia and Tuva]: Sibirskii Nauchno-Issled. Inst. Geologii Geofizii, i Mineralogo Syr 'ya Trudy, Paleontogiya i Stratigrafiya, no. 42, 270 p., 12 figs., 52 pls.

Tidwell, W. D., 1967, Flora of Manning Canyon Shale. Part 1. A lowermost Pennsylvanian flora from the Manning Canyon Shale, Utah, and its stratigraphic significance: Brigham Young Univ. Geol. Studies, v. 14, p. $3-66$, figs. $1-5$, pls. $1-10$.

Tidwell, W. D., Rushforth, S. R., and Reveal, J. L., 1967, A new Cretaceous fern genus from Utah and Colorado: Brigham Young Univ. Geol. Studies, v. 14, p. 237-240, 1 fig., 6 pls.

Townrow, J. A., 1967a, On a conifer from the Jurassic of East Antarctica: Royal Soc. Tasmania Papers and Proc., v. 1, p. 137-147, 5 figs.; 1 pl. 1967b, On Rissikia and Matia podocarpaceous conifers from the lower Mesozoic of southern lands. Royal Soc. Tasmania Papers and Proc., v. 101, p. 103-136, 15 figs., 2 pls.

Trivedi, B. S.,, and Ambwani, K., 1971, On the structure of Nymphaeocaulon intertrappeum gen. et sp. nov. from the Deccan Intertrappean series of 
Mohgaonkalon (M. P.), India: Palaeontographica, Abt. B, v. 133, pt. 4-6, p. 129-136, 3 figs., pls. 44,45 .

Trivedi, B. S., and Chaturvedi, S. K., 1972, Voyrioseminites magnus gen. et sp. nov., a fossil seed from Tertiary coal of Malaya: Geophytology (Lucknow, India), v. 1, no. 2, (1971) p. 161-164, 2 figs., 1 pl.

Trivedi, B. S., and Verma, C. L., 1971 The structure of pseudostem and root of Cannaites intertrappea gen. et sp. nov. from the Deccan Intertrappean beds of Madhya Pradesh, India: Palaeontographica, Abt. B, v. 132 , pt. 5-6, p. 174-185, figs. $1-4$; pls. 29 , 30, figs. $1-12$.

1972, Occurrence of Heliconiaites mohgaonensis gen. et sp. nov. from the early Eocene of Deccan Intertrappean Series, M. P., India: Palaeontographica, Abt. B, v. 139, pt. 5-6, p. 73-82, 4 figs., pls. 46-48, figs. 1-16.

Tyroff, Helmut, 1966, Die Algen und Koniferen des Zechsteins von Büdingen (Wetterau) : Senckenbergiana Lethaea, v. 47, no. 3, p. 253-277, 4 figs., pls. $32-37$.

Unger, Franz, 1841-47, Beiträge zur Flora der Vorwelt-Chloris Protogaea: Leipzig, p. i-cx, 1-150, 50 pls. (In 10 parts pt. 2, p. 17-44, pls. $6-15,1842$ ) 1850 , Genera et species plantarum fossilium: Vienna, $627 \mathrm{p}$.

Vakhrameev, V. A., Novye Mezozoiskie paporotniki, in Rasteniya Mezozoya [New Mesozoic ferns, in Mesozoic plants]: Akad. Nauk SSSR, Geol. Inst., Trudy, No. 191, p. 7-15, 4 figs., 4 pls.

Varma, C. P., 1968, On a collection of leaf impressions from Hardwar beds Shivalik Formation) near Hardwar, Uttar Pradesh: Paleont. Soc. India, Prem Nath Ganju Memorial Volume, v. 5-9 (1960-64) p. 83-88, 2 figs., $1 \mathrm{pl}$.

Vasil'yev, V. N., 1967, Novyy rod semeistva Trapaceae [New genus of the family Trapaceae]: Akad Nauk SSSR, Palaeont. Zhur., 1967, no. 2, p. 107-112, pl. 8, figs. 1-6.

Vladimirovich, V. P., 1972, O srednetriasovoy flore Priurala, in Platformennye obrazovaniya Urala. Sbornik po Stratigrafii, No. 17 [On Middle Triassic flora of Orenburg in the Ural region, in Platformal structures of the Urals. Collected Stratigraphic Papers, no. 17]: Akad. Nauk, SSSR, Ural'skii Nauchnyi Tsentr. Inst. Geologii i Geokhimi, Trudy, no. 96. p. $87-98,2$ pls.

Vladimirovich, V. P., and others, 1967, Stratigrafiya Triaskvykh otlozheniy sredney Sibiri, in Stratigrafiya mezozoya i kaynozoya sredney Sibiri [Stratigraphy of Triassic deposits of central Siberia, in Stratigraphy of the Mesozoic and Cenozoic of central Siberia]: Novosibirsk, Akad. Nauk SSSR, Sibirskoye Otdel., p. 7-30, 12 figs.

Vogellehner, Dieter, 1967, Zur Anatomie und Phylogenie Mesozoischer Gymnospermenhölzer, 5. Prodromus zu Einer Monographie der Protopinaceae: Palaeontographica, Abt. B, v. 121 , pts. $1-3$, p. $30-51$.

1968, Zur Anatomie und Phylogenie Mesozoischer Gymnospermenhölzer, 7. Prodromus zu einer Monographie der Protopinaceae, II, Die Protopinoiden Hölzer des Jura: Palaeontographica, Abt. B, v. 124, pts. 4-6, p. 125162.

Vologdin, A. G., 1962, Drevneyshie vodorosli SSSR. Chast' 1. Vodorosli Siniya. Chast' 2. Vodorosli Kembriya i Ordovika. [The most ancient algae of the U.S.S.R. Part 1, Sinian algae. Part 2. Cambrian and Ordovician algae]: Moscow, Akad. Nauk SSSR, 656 p. [In 2 parts with continuous pagination. Each part has separate sets of text-figs, and plates. Pt. 1. text-figs. 1-95, pls. $1-80$; Pt. 2. text-figs. $1-40$, pls. 1-46.] 
1969, Sinezelenya vodorosli Kembriya uscheliya Ulug-Shangan, Tuva [Cambrian cyanophyceans from Ulug-Shangan gorge, Tuva]: Akad. Nauk SSSR Doklady, v. 188, no. 6, p. 1377-1379, 4 figs.

1970, Ostatki organizmov iz shungitov, Dokembriya Karelii [Organic remains from shungites in the Precambrian of Karelia]: Akad. Nauk SSSR, Doklady, v. 193, no. 5, p. 1163-1166, figs. 1a, b, v.

Vologdin, A. G., and Drosdova, N. A., 1969a, Kotkrytiyu vodoroslei semenistva Rivulariaceae $v$ pozdnei dokembrii [A discovery of algae of the family Rivulariaceae in the late Precambrian]: Akad. Nauk SSSR Doklady, v. 187, no. 5, p. 1162-1163, fig. I: 1-4 (opposite p. 1161).

$-1969 b$, Novye sineselenye vodorosli Dokembriiskogo vozrasta iz Batenevskogo Kriyashe [New Precambrian cyanophyceans from Batenev ridge]: Akad. Nauk SSSR Doklady, v. 187, no. 2, p. 440-442, fig. I:1-5. -1969c, Vodorosli Sem. Gloeocapsaceae $\mathrm{v}$ osadkakh Dokembriya Batenevskogo Kryazha [Algae of the Family Gloeocapsaceae in the Precambrian of Batenev ridge] : Akad. Nauk SSSR Doklady, v. 186, no. 67, p. 14191421, fig. I:1-6.

Vologdin, A. G., and Kochetkov, O. S., 1966, Ob otkrytii ostatkov gigantiskikh Sifoney $\mathrm{v}$ drevnikh sloyakh Timanskogo Kryazha [The discovery of gigantic Siphonea in ancient strata of the Timanian mountain range]: Akad. Nauk SSSR Doklady, v. 169 , no. 3, p. 672-675, figs. 1-4.

Vologdin, A. G., and Strygin, A. I., 1969, Otkrytie ostatkov organismov v verkhneiy svite Krivorozhskoy serii Dokembriya Ukrainy [The discovery of organic remains in the upper suite of the Krivorozhsk series in the Precambrian of the Ukraine]: Akad. Nauk. SSSR Doklady, v. 188, no. 2, p. 446-449, 2 figs.

Walter, M. R., 1972, Stromatolites and the biostratigraphy of the Australian Precambrian and Cambrian. Special Papers in Palaeontology, II: London, Palaeont. Assoc., 268, p. 55 figs.; 34 pls.

Walthur, Harold, 1967, Ergänzungen zur Flora von Seifhennersdorf (Sachsen), I. Teil: Dresden Staatliches Mus. Mineralogie Geologie Abh., v. 12, p. 259277, 4 figs., 2 pls.

Watson, Joan, 1969, A revision of the English Wealden flora; I. CharalesGinkgoales: British Mus. (Natural History) Bull. Geology, v. 17, no. 5, p. 207-254, 64 figs., 6 pls.

Weyland, Herman, und Berendt, Werner, 1968, Neue Pflanzen aus den Brandenberg-Schichten des Mittledevons von Wuppertal-Elberfeld: Palaeontographica, Abt. B, v. 122, pt. 4-6, p. 171-182, 4 figs.; pls. 27-30.

Wille, Wolfgang, 1970, Plaesiodictyon mosellanum n.g., n.s., eine mehrzellige Grunalge aus dem unteren Keuper von Luxemburg: Neues Jahrb. Geologie Paleontologie, Monatsh., no. 5, p. 283-310, 15 figs.

Wolfe, J. A., 1968, Paleogene biostratigraphy of nonmarine rocks in King County, Washington: U.S. Geol. Survey Prof. Paper 571, 33 p., 5 pls.

Wray, J. L., 1967, Upper Devonian calcareous algae from the Canning basin, Western Australia: Colorado Sch. Mines Prof. Contr., no. 3, 76 p., 18 figs., 11 pls.

Yoshida, Riuiti, 1970, Nôvo gênero de Conífera da Formação Estrada Nova, norte do Estado de Santa Catarina: Brazil Div. Geologia e Mineralogia Bol. 249, p. 5-17, 4 pls.

Yurina A. L., 1969, Devonskaya flora Tsentral'nogo Kazakhstana, in Materialy po geologii Tsentral'nogo Kazakhstana, t. 8 [Devonian flora of central 
54 INDEX OF GENERIC NAMES OF FOSSIL PLANTS, 1966-1973

Kazakhstan, in Geologic records of central Kazakhstan, v. 8]: Moscow, Moskovskiy Gosudarstvennyy Univ., 207 p., 38 figs., 30 pls.

Zalessky, M. D., 1944, Uber drei neue Pflanzenreste aus den stephanischen und amadokischen Ablagerungen des Donezbeckens: Neues Jahrb. Mineralogie, Geologie, Paläontologie Monatsh, Abt. B, no. 7/9. p. 188-192, 3 figs.

H.S. GOVERNMENT PRINTING OFFICE: $19750-585-474 / 100$ 




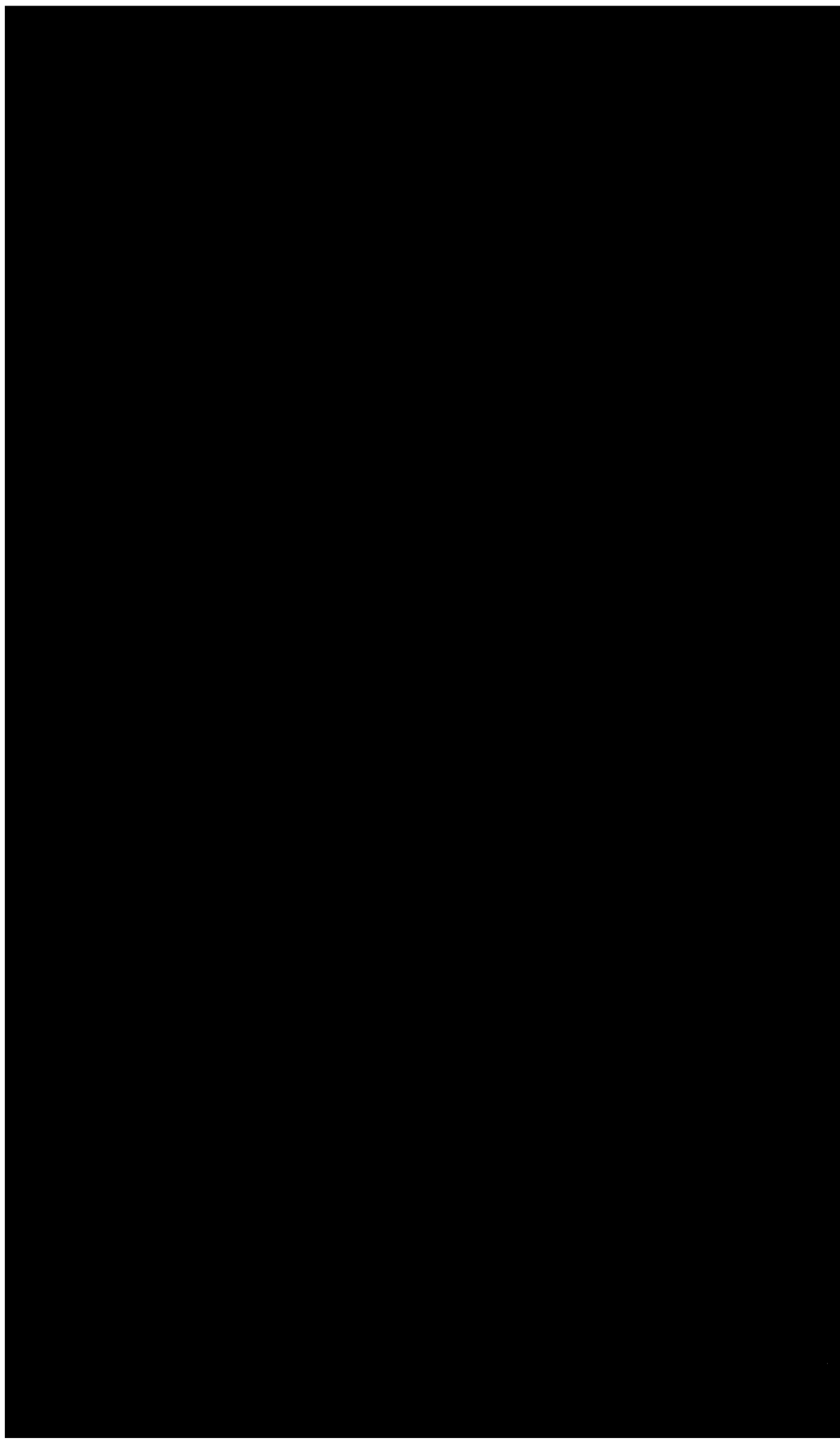




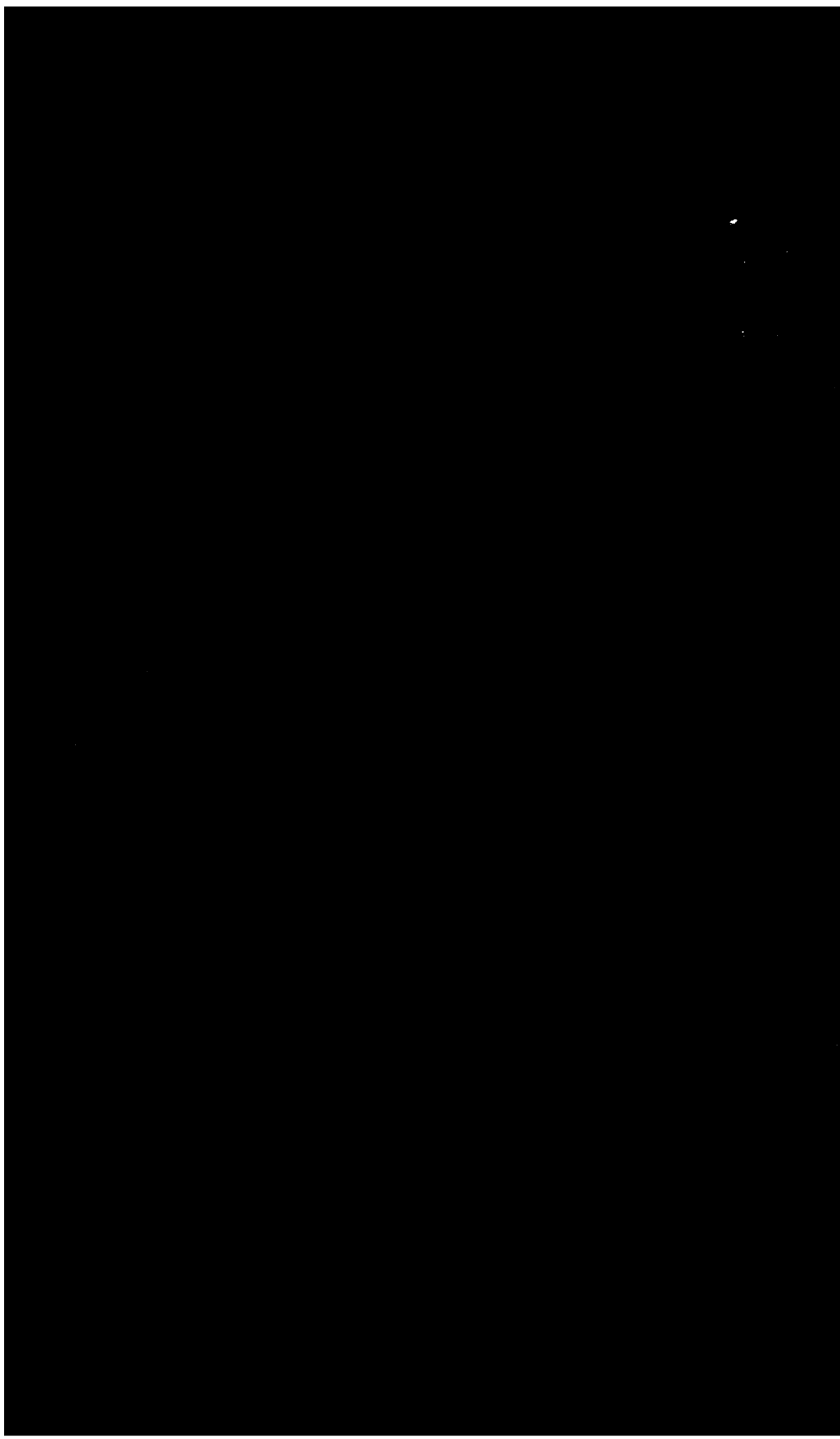

